Supporting Information for

\title{
Reagent-Controlled $\alpha$-Selective Dehydrative Glycosylation of 2,6-Dideoxy sugars: Construction of the Arugomycin Tetrasaccharide
}

Joseph R. Romeo, Luca McDermott, Clay S. Bennett

Department of Chemistry, Tufts University, 62 Talbot Ave. Medford, MA 02155, USA

\section{Contents}

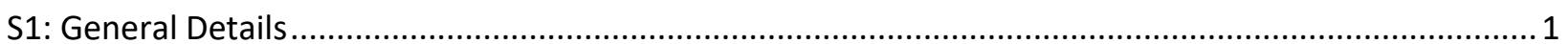

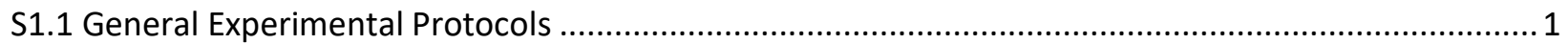

S1.2 Preparation of 1,1,2-trichloroethylene glycosylation solvent …................................................ 2

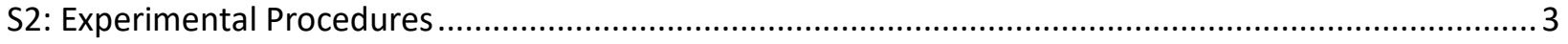

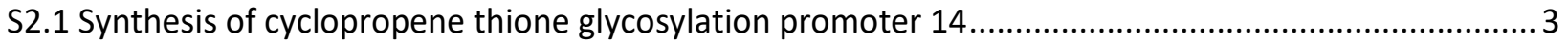

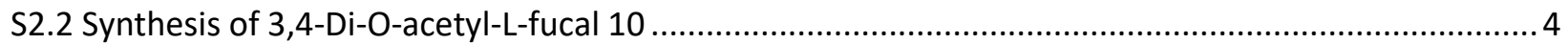

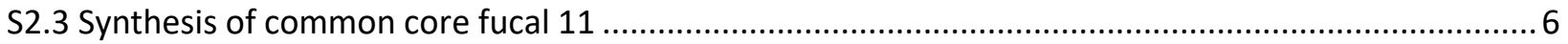

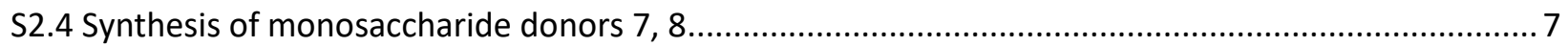

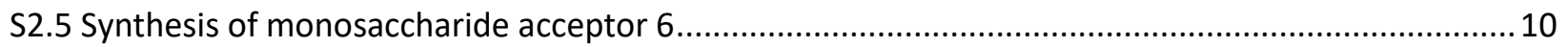

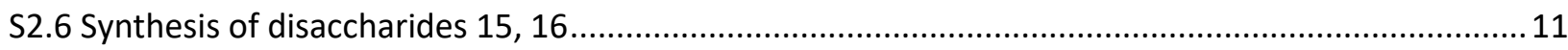

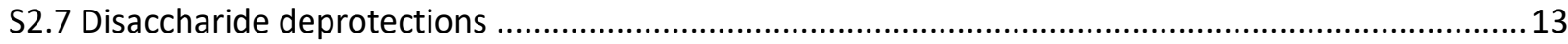

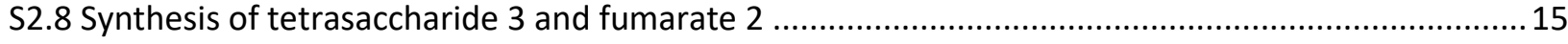

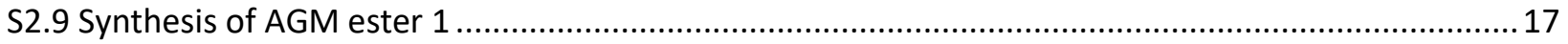

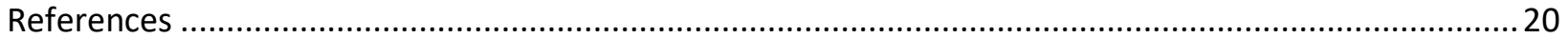

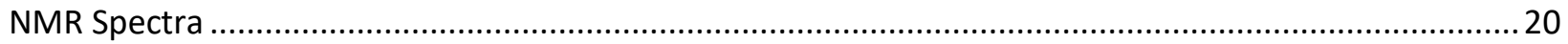

S1: General Details

\section{S1.1 General Experimental Protocols}

Prior to running glycosylation reactions, all solid reagents were dried by azeotropic removal of water using toluene and a rotary evaporator then set under reduced pressure $16 \mathrm{~h}$ in flame-dried flasks before use, with the exception of the para-methoxyphenol (PMP) acceptor. All reactions were performed under inert argon atmosphere, unless otherwise noted. Solvents for glycosylation reactions were dried through a commercial solvent purification system immediately 
prior to use, with the exception of 1,1,2-trichloroethylene (section S1.2). Isobutylene oxide was dried over sodium sulfate for one hour, redistilled under atmospheric pressure, and then stored under Argon in a flame-dried flask prior to use. All other chemicals were purchased at the highest possible quality and used as received. Temperatures below $0{ }^{\circ} \mathrm{C}$ for tetrasaccharide glycosylations were maintained using a Neslab CC100 immersion cooler. Manual flash column chromatography was performed on 230-400 mesh silica gel. Automated flash column chromatography was performed on a Smart Flash EPCLC W-Prep 2XY Dual Channel Automated Flash Chromatography System with an additional ELSD detector, provided by Yamazen Corporation (Japan). Analytical and preparative thin layer chromatography was carried out on silica gel 60 F-254 plates. Products were visualized using UV or by staining with either $5 \%$ aqueous sulfuric acid or potassium permanganate solution.

NMR spectra were recorded on an NMR spectrometer at $500 \mathrm{MHz}$ for $1 \mathrm{H}$ NMR and 126 $\mathrm{MHz}$ for ${ }^{13} \mathrm{C}$ NMR. Chemical shifts are reported in ppm relative to TMS (for $1 \mathrm{H} \mathrm{NMR} \mathrm{in} \mathrm{CDCl} 3$ ) or residual solvent peaks $\mathrm{CDCl}_{3}$ (for ${ }^{13} \mathrm{C} \mathrm{NMR}$ in $\mathrm{CDCl}_{3}$ ), $\mathrm{CD}_{2} \mathrm{Cl}_{2}$ (for ${ }^{1} \mathrm{H} / \mathrm{C}^{13} \mathrm{NMR}$ in $\mathrm{CD}_{2} \mathrm{Cl}_{2}$ ), and $\mathrm{C}_{6} \mathrm{D}_{6}$ (for ${ }^{1} \mathrm{H} /{ }^{13} \mathrm{C}$ NMR in $\mathrm{C}_{6} \mathrm{D}_{6}$. For $1 \mathrm{H}$ NMR spectra, data are reported as follows: $\delta$ shift, multiplicity (s = singlet, $\mathrm{m}=$ multiplet, $\mathrm{t}=$ triplet, $\mathrm{d}=$ doublet, $\mathrm{q}=$ quartet, $\mathrm{dd}=$ doublet of doublets, $\mathrm{dq}=$ doublet of quartets, $\mathrm{ddd}=$ doublet of doublet of doublets), coupling constants are reported in $\mathrm{Hz}$. Low resolution mass spectra (LRMS) were recorded using an ESI-MS with an additional APCI source. High-resolution mass spectra (HRMS) were obtained on ElectroSpray lonization (ESI) on a Waters Qtof Premier instrument in the positive mode or Fourier Transform Ion Cyclotron Resonance Mass Spectrometer (FTICR- MS) with direct analysis in real time (DART) ionization source. Optical rotations were measured at $589 \mathrm{~nm}$ in a $5 \mathrm{~cm}$ cell at room temperature.

\section{Chemical abbreviations:}

DTBMP: 2,6-di-tert-butyl-4-methyl-pyridine

DMAP: N,N-dimethyl-4-aminopyridine

DIPEA: N,N-Diisopropylethylamine

DMF: N,N-dimethylformamide

TTBP: 2,4,6-tri-tert-butylpyrimidine

DTBMP: 2,6-Di-tert-butyl-4-methylpyridine

ADB: 4-Allyl-1,2-dimethoxybenzene

NapBr: 2-(Bromomethyl)naphthalene

IBO: Isobutylene oxide

DDQ: 2,3-dichloro-5,6-dicyano-1,4-benzoquinone

PMP: p-methoxyphenol

TCE: 1,1,2-trichloroethylene

$\mathrm{EDCl} \bullet \mathrm{HCl}$ : N-(3-Dimethylaminopropyl)-N'-ethylcarbodiimide hydrochloride

\section{S1.2 Preparation of 1,1,2-trichloroethylene glycosylation solvent}

$4 \AA ̊$ molecular sieves that were dried overnight in an oven $\left(160^{\circ} \mathrm{C}\right)$ were added to a flamedried round-bottom flask and microwaved in a conventional microwave oven 5 times for 20 second intervals. The sieves flask was then flamed dried under high vacuum, cooled to rt, then 
backfilled with argon. TCE was added directly into the sieves flask and stored under argon overnight prior to glycosylation. The storage flask was wrapped in foil to prevent light exposure.

S2: Experimental Procedures

S2.1 Synthesis of cyclopropene thione glycosylation promoter 14
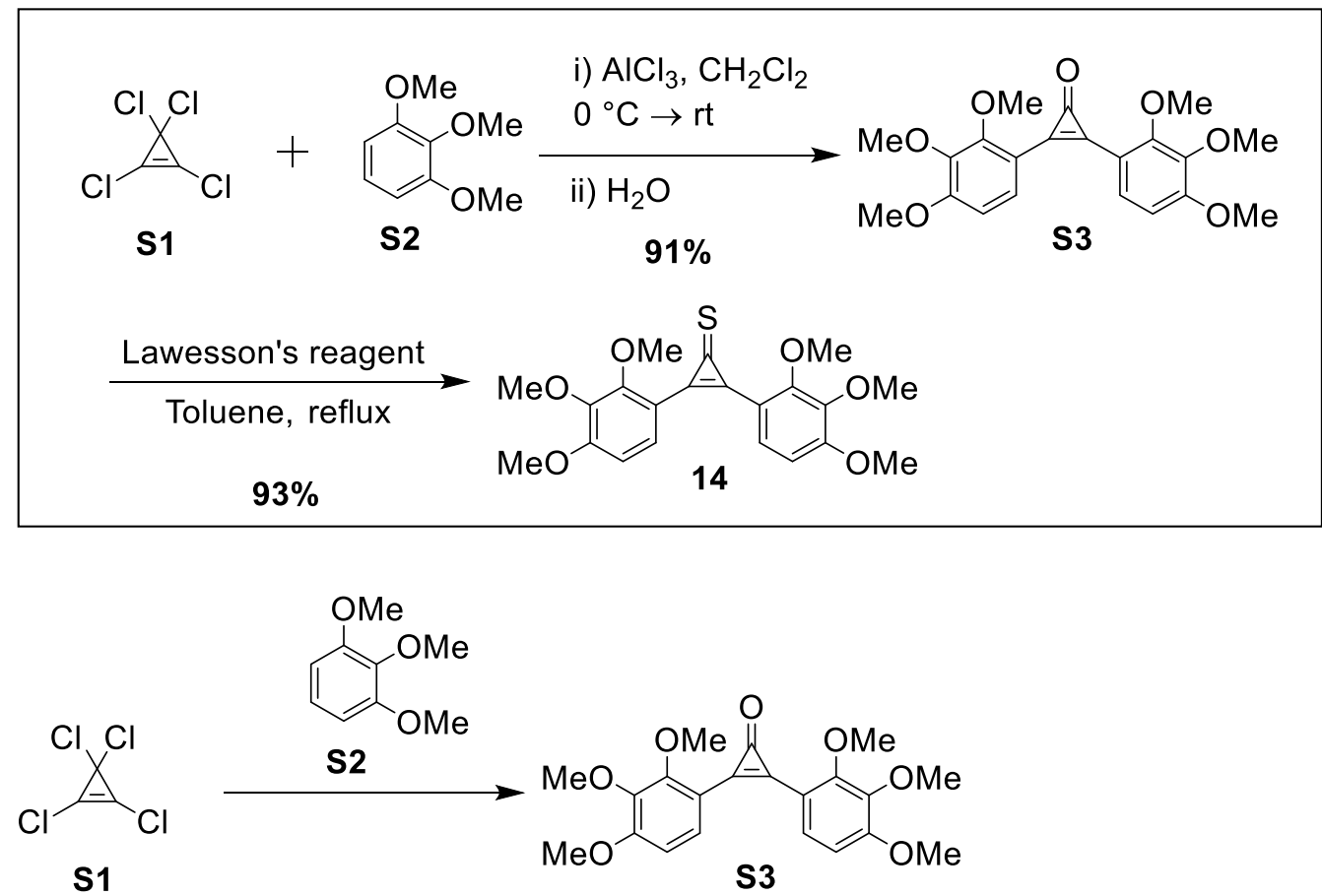

\section{2,3-bis(2,3,4-trimethoxyphenyl)cycloprop-2-en-1-one (S3)}

To a solution of aluminum chloride $(12 \mathrm{~g}, 90 \mathrm{mmol})$ in $392 \mathrm{~mL} \mathrm{CH} \mathrm{Cl}_{2}$ was added tetrachlorocyclopropene $(\mathbf{S 1}, 10 \mathrm{~mL}, 82 \mathrm{mmol})$. The solution was cooled to $0{ }^{\circ} \mathrm{C}$, and $1,2,3-$ trimethoxybenzene (S2, $27.6 \mathrm{~g}, 164 \mathrm{mmol}$ ) was added in two portions 2.5 minutes apart, after which the reaction was allowed to warm to rt. After 4 hours, the reaction was again cooled to 0 ${ }^{\circ} \mathrm{C}$ and quenched with $1.2 \mathrm{~L} \mathrm{H}_{2} \mathrm{O}$. The organic layer was then washed with $\mathrm{H}_{2} \mathrm{O}(2 \mathrm{x})$, saturated sodium bicarbonate $(1 \mathrm{x})$, and brine (1x). The organic layer was then dried over sodium sulfate, filtered through cotton, and concentrated under reduced pressure. The crude material was then purified by flash chromatography (30\% to $60 \%$ ethyl acetate:hexanes, followed by $0 \%$ to $20 \%$ methanol: $\mathrm{CH}_{2} \mathrm{Cl}_{2}$ ) and dried on high vacuum overnight to afford aryl cyclopropenone $\mathbf{S 3}(28.95 \mathrm{~g}$, $91 \%)$ as a beige amorphous solid. Spectroscopic data was in agreement with previously reported data. ${ }^{1}$

\footnotetext{
${ }^{1} \mathrm{H}$ NMR $\left(500 \mathrm{MHz}, \mathrm{CDCl}_{3}\right) \delta 7.77(\mathrm{~d}, J=8.7 \mathrm{~Hz}, 2 \mathrm{H}), 6.83(\mathrm{~d}, J=8.8 \mathrm{~Hz}, 2 \mathrm{H}), 4.01(\mathrm{~s}, 6 \mathrm{H}), 3.96(\mathrm{~s}, 6 \mathrm{H}), 3.94(\mathrm{~s}, 6 \mathrm{H})$.

${ }^{13} \mathrm{C} \mathrm{NMR}\left(126 \mathrm{MHz}, \mathrm{CDCl}_{3}\right) \delta 157.7,155.6,154.8,142.5,141.2,128.6,112.8,107.7,62.5,61.3,56.4$.
} 
<smiles>COc1ccc(C2=C(c3ccc(OC)c(OC)c3OC)C(c3ccc(OC)c(OC)c3OC)C2=O)c(OC)c1OC</smiles>

\section{2,3-bis(2,3,4-trimethoxyphenyl)cycloprop-2-ene-1-thione (14)}

In a flame dried flask, compound $\mathbf{S 3}$ (17 g, $44 \mathrm{mmol}$ ) was suspended in $179 \mathrm{~mL}$ dry toluene, and Lawesson's reagent ( $9 \mathrm{~g}, 22 \mathrm{mmol}$ ) was added. The reaction was brought to reflux using an oil bath and stirred for two hours. The reaction was then allowed to cool to rt before direct concentration under reduced pressure. The crude material was then purified by flash chromatography (30\% to $80 \%$ ethyl acetate:hexanes) and dried on high vacuum to afford thione glycosylation promoter 14 (16.47 g, 93\%) as a neon-yellow amorphous solid. Spectroscopic data was in agreement with previously reported data. ${ }^{2}$

${ }^{1} \mathrm{H}$ NMR $\left(500 \mathrm{MHz}^{\mathrm{CDCl}}{ }_{3}\right) \delta 8.12(\mathrm{~d}, J=8.7 \mathrm{~Hz}, 2 \mathrm{H}), 6.87(\mathrm{~d}, J=8.8 \mathrm{~Hz}, 2 \mathrm{H}), 4.08(\mathrm{~s}, 6 \mathrm{H}), 3.99(\mathrm{~s}, 6 \mathrm{H}), 3.95(\mathrm{~s}, 6 \mathrm{H})$.

${ }^{13} \mathrm{C} \mathrm{NMR}\left(126 \mathrm{MHz}, \mathrm{CDCl}_{3}\right) \delta 175.9,158.9,155.2,148.0,142.5,129.9,111.6,107.9,62.9,61.3,56.5$.

S2.2 Synthesis of 3,4-Di-O-acetyl-L-fucal 10
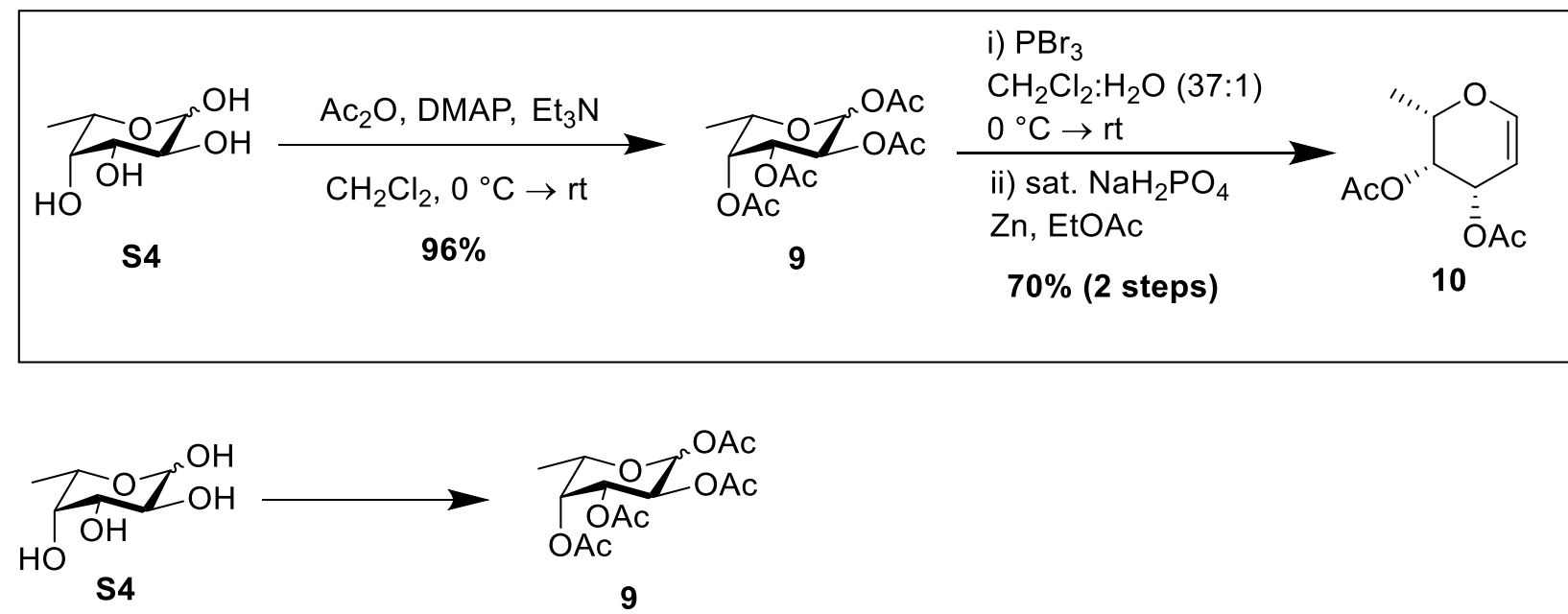

peracetylated L-Fucose (9)

L-Fucose (S4, $4 \mathrm{~g}, 24.4 \mathrm{mmol}$ ) and DMAP (294 mg, $2.4 \mathrm{mmol}$ ) were suspended in $104 \mathrm{~mL}$ $\mathrm{CH}_{2} \mathrm{Cl}_{2}$ and the mixture was cooled to $0{ }^{\circ} \mathrm{C}$. Triethylamine $(27.2 \mathrm{~mL}, 194.8 \mathrm{mmol})$ and acetic anhydride $(13.8 \mathrm{~mL}, 146 \mathrm{mmol})$ were then added slowly at $0{ }^{\circ} \mathrm{C}$. The reaction was stirred for 5 minutes and then removed from the ice bath and allowed to warm to rt. After 2.5 hours, the reaction was concentrated directly under reduced pressure. The crude material was then purified 
by flash chromatography ( $15 \%$ to $50 \%$ ethyl acetate:hexanes) and dried on high vacuum overnight to afford peracetylated L-Fucose $\mathbf{9}(7.78 \mathrm{~g}, 96 \%)$ as a beige amorphous solid.

${ }^{1} \mathrm{H}$ NMR $\left(500 \mathrm{MHz}, \mathrm{CDCl}_{3}\right) \delta 6.34(\mathrm{~d}, J=2.9 \mathrm{~Hz}, 1 \mathrm{H}), 5.38-5.29(\mathrm{~m}, 3 \mathrm{H}), 4.28(\mathrm{q}, J=7.1,6.5 \mathrm{~Hz}, 1 \mathrm{H}), 2.18(\mathrm{~s}, 3 \mathrm{H})$, $2.15(\mathrm{~s}, 3 \mathrm{H}), 2.02(\mathrm{~s}, 3 \mathrm{H}), 2.01(\mathrm{~s}, 3 \mathrm{H}), 1.16(\mathrm{~d}, J=6.5 \mathrm{~Hz}, 3 \mathrm{H})$.

${ }^{13} \mathrm{C} \mathrm{NMR}\left(126 \mathrm{MHz}, \mathrm{CDCl}_{3}\right) \delta 170.5,170.2,169.9,169.1,90.0,70.6,67.8,67.3,66.5,20.9,20.7,20.6,20.6,15.9$.

LRMS (ESI) m/z: (M+Na) ${ }^{+}$Calcd. $\mathrm{C}_{14} \mathrm{H}_{20} \mathrm{O}_{9} \mathrm{Na} 355.1005$; Found 355.1800

HRMS (ESI) m/z: $(\mathrm{M}+\mathrm{Na})^{+}$Calcd. $\mathrm{C}_{14} \mathrm{H}_{20} \mathrm{O}_{9} \mathrm{Na} 355.1005$; Found 355.0991

$[\alpha]_{\lambda}{ }^{24}\left(\mathrm{c}=1.8, \mathrm{CH}_{2} \mathrm{Cl}_{2}\right):-0.944$

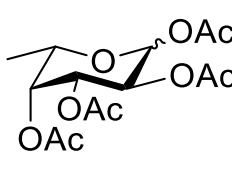

9<smiles>CC(=O)O[C@@H]1C=CO[C@@H](C)[C@H]1OC(C)=O</smiles>

10

\section{3,4-Di-O-acetyl-L-fucal (10)}

Compound 9 (7.78 g, $23.41 \mathrm{mmol}$ ) was dissolved in $94 \mathrm{~mL}$ of $\mathrm{CH}_{2} \mathrm{Cl}_{2}$ and $2.5 \mathrm{~mL} \mathrm{H} \mathrm{H}_{2} \mathrm{O}$ and the solution was cooled to $0{ }^{\circ} \mathrm{C} . \mathrm{PBr}_{3}(2.9 \mathrm{~mL}, 39.8 \mathrm{mmol})$ was added slowly to the reaction, which was removed from the ice bath after 10 minutes of stirring and allowed to warm to rt. After 1.75 hours of stirring, the reaction was cooled to $0{ }^{\circ} \mathrm{C}$ and quenched under an Argon balloon with 100 $\mathrm{mL}$ saturated sodium bicarbonate solution added slowly. The organic phase was then washed with saturated sodium bicarbonate $(2 \mathrm{x})$, and the pooled aqueous layers with back-extracted with $\mathrm{CH}_{2} \mathrm{Cl}_{2}(3 \mathrm{x})$. The resulting organic layer was then washed with brine (1x), dried over sodium sulfate, filtered through cotton, and concentrated under reduced pressure.

The resulting crude oil was then dissolved in $47 \mathrm{~mL}$ of ethyl acetate and $94 \mathrm{~mL}$ saturated aqueous $\mathrm{NaH}_{2} \mathrm{PO}_{4}$ solution. Zinc powder (19.15 g, $\left.293 \mathrm{mmol}\right)$ was then added and the reaction was stirred vigorously at room temperature. After 20 hours, the reaction was filtered through a 4" pad of celite with ethyl acetate and $\mathrm{H}_{2} \mathrm{O}$. The aqueous layer of the filtrate was extracted with ethyl acetate $(3 x)$, and the pooled organic layers were washed with $\mathrm{H}_{2} \mathrm{O}(1 \mathrm{x})$ and brine $(1 \mathrm{x})$. The organic layer was then dried over sodium sulfate, filtered through cotton, and concentrated under reduced pressure. The crude material was then purified by flash chromatography (10\% to $25 \%$ ethyl acetate:hexanes) and dried on high vacuum overnight to afford 3,4-Di-O-acetyl-L-fucal 10 (3.51 g, 70\%) as a white amorphous solid. Spectroscopic data was in agreement with previously reported data. ${ }^{3}$

${ }^{1} \mathrm{H} \mathrm{NMR}\left(500 \mathrm{MHz}, \mathrm{CDCl}_{3}\right) \delta 6.46(\mathrm{dd}, J=6.4,2.0 \mathrm{~Hz}, 1 \mathrm{H}), 5.61-5.55(\mathrm{~m}, 1 \mathrm{H}), 5.32-5.26(\mathrm{~m}, 1 \mathrm{H}), 4.64(\mathrm{dt}, J=6.3$, $2.0 \mathrm{~Hz}, 1 \mathrm{H}), 4.22(\mathrm{q}, J=6.6 \mathrm{~Hz}, 1 \mathrm{H}), 2.16(\mathrm{~s}, 3 \mathrm{H}), 2.02(\mathrm{~s}, 3 \mathrm{H}), 1.28(\mathrm{~d}, J=6.7 \mathrm{~Hz}, 3 \mathrm{H})$.

${ }^{13} \mathrm{C}$ NMR $\left(126 \mathrm{MHz}, \mathrm{CDCl}_{3}\right) \delta 170.8,170.5,146.3,98.4,71.7,66.5,65.2,21.0,20.8,16.7$. 
S2.3 Synthesis of common core fucal 11

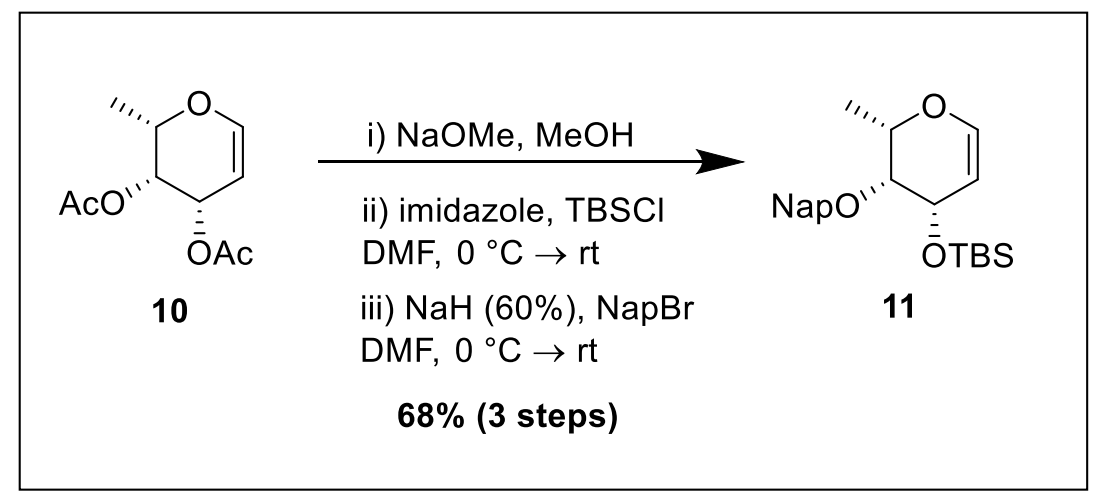

\section{3-0-(tert-butyldimethylsilyl)-4-0-(2-napthylmethyl)-L-fucal (11)}

To a solution of compound $10(3.51 \mathrm{~g}, 16.39 \mathrm{mmol})$ in $26.4 \mathrm{~mL} \mathrm{MeOH}$ was added solid sodium methoxide ( $266 \mathrm{mg}, 4.92 \mathrm{mmol}$ ) and the reaction was stirred at room temperature. After 12 hours of stirring, the reaction was adjusted to $\mathrm{pH} 6$ with dropwise addition of acetic acid and concentrated directly under reduced pressure. The crude material was then azeotroped twice with toluene and dried on high vacuum for 1.5 hours.

The resulting crude oil and imidazole $(2.23 \mathrm{~g}, 32.78 \mathrm{mmol})$ were then dissolved in $35 \mathrm{~mL}$ DMF and the reaction was cooled to $0{ }^{\circ} \mathrm{C}$. tert-butyldimethylsilyl chloride was then added and the reaction was stirred from $0{ }^{\circ} \mathrm{C}$ to rt. After 1.25 hours of stirring the reaction was diluted with water, and the aqueous phase was extracted with ethyl acetate $(3 x)$. The pooled organic layers were then washed with a $1 \mathrm{M} \mathrm{LiCl}$ solution $(3 \mathrm{x})$ and brine $(2 \mathrm{x})$, dried over sodium sulfate, filtered through cotton, and concentrated to an oil under reduced pressure.

The above crude oil was then redissolved in $35 \mathrm{~mL}$ DMF and the solution was cooled to 0 ${ }^{\circ} \mathrm{C}$. Sodium hydride $(60 \% \mathrm{wt}, 1.31 \mathrm{~g}, 32.8 \mathrm{mmol})$ was then added at $0{ }^{\circ} \mathrm{C}$ and the reaction was stirred for 25 minutes. A solution of 2-napthylmethyl bromide $(7.25 \mathrm{~g}, 32.8 \mathrm{mmol})$ in $11.2 \mathrm{~mL}$ DMF was then added to the reaction by cannulation, and the reaction was stirred at $0{ }^{\circ} \mathrm{C}$ for 10 minutes prior to removal of the ice bath. After 16 hours of additional stirring, the reaction was again cooled to $0{ }^{\circ} \mathrm{C}$ and quenched with saturated ammonium chloride solution. The reaction was further diluted with water, and the aqueous phase was then extracted with diethyl ether (3x). The pooled organic phase was then washed with $1 \mathrm{M} \mathrm{LiCl}$ solution $(4 \mathrm{x})$ and brine $(1 \mathrm{x})$, dried over sodium sulfate, filtered through cotton, and concentrated under reduced pressure. The crude material was then purified by flash chromatography ( $0 \%$ to $2 \%$ acetone:hexanes) to afford common core L-fucal 11 (4.28 g, 68\%) as a white amorphous solid.

${ }^{1} \mathrm{H}$ NMR $\left(500 \mathrm{MHz} \mathrm{CDCl}_{3}\right) \delta 7.85-7.78(\mathrm{~m}, 4 \mathrm{H}), 7.55(\mathrm{dd}, J=8.5,1.7 \mathrm{~Hz}, 1 \mathrm{H}), 7.50-7.41(\mathrm{~m}, 2 \mathrm{H}), 6.32(\mathrm{dd}, J=6.3$, $1.8 \mathrm{~Hz}, 1 \mathrm{H}), 5.18(\mathrm{~d}, J=12.1 \mathrm{~Hz}, 1 \mathrm{H}), 4.84(\mathrm{~d}, J=12.2 \mathrm{~Hz}, 1 \mathrm{H}), 4.65(\mathrm{dt}, J=6.2,2.0 \mathrm{~Hz}, 1 \mathrm{H}), 4.62-4.58(\mathrm{~m}, 1 \mathrm{H}), 4.06$ $(q, J=6.6 \mathrm{~Hz}, 1 \mathrm{H}), 3.59-3.54(\mathrm{~m}, 1 \mathrm{H}), 1.24(\mathrm{~d}, J=6.6 \mathrm{~Hz}, 3 \mathrm{H}), 0.96(\mathrm{~s}, 9 \mathrm{H}), 0.16(\mathrm{~d}, J=4.8 \mathrm{~Hz}, 6 \mathrm{H})$.

${ }^{13} \mathrm{C}$ NMR $\left(126 \mathrm{MHz}, \mathrm{CDCl}_{3}\right) \delta 143.7,136.3,133.2,133.0,127.9,127.9,127.7,126.8,126.4,125.9,125.7,103.0$, $75.2,74.2,72.9,66.8,26.0,25.9,18.2,16.7,-4.5,-4.7$. 
LRMS (ESI) m/z: (M+Na)+ Calcd. $\mathrm{C}_{23} \mathrm{H}_{32} \mathrm{O}_{3} \mathrm{SiNa} 407.2018$; Found 407.36

HRMS (ESI) m/z: (M+Na)+ Calcd. $\mathrm{C}_{23} \mathrm{H}_{32} \mathrm{O}_{3} \mathrm{SiNa}$ 407.2018; Found 407.2000

$[\alpha]_{\lambda}{ }^{24}\left(\mathrm{c}=0.75, \mathrm{CH}_{2} \mathrm{Cl}_{2}\right): 0.216$

S2.4 Synthesis of monosaccharide donors 7, 8
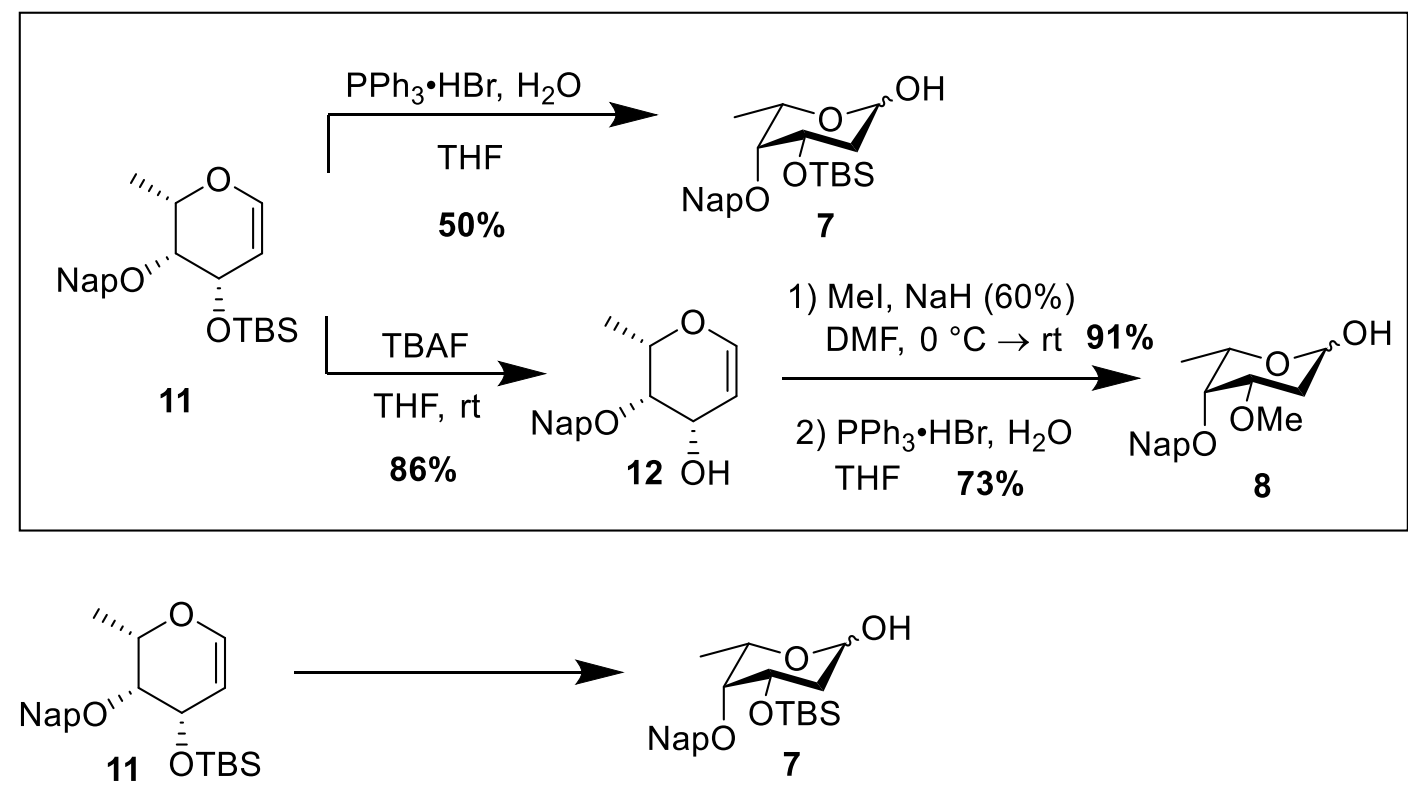

\section{2-deoxy-3-O-(tert-butyldimethylsilyl)-4-0-(2-napthylmethyl)- $\alpha / \beta$-L-fucopyranoside (7)}

To a solution of fucal 11 (9.99 $\mathrm{g}, 35.1 \mathrm{mmol})$ in THF (197 mL) was added triphenylphosphine hydrobromide $(2.41 \mathrm{~g}, 7.02 \mathrm{mmol})$ at rt. After 10 minutes of stirring, $\mathrm{H}_{2} \mathrm{O}$ was added ( $3.2 \mathrm{~mL}, 176 \mathrm{mmol}$ ) and the reaction was stirred at $\mathrm{rt}$ for one hour. The reaction was then quenched with saturated sodium bicarbonate solution, and the aqueous phase was extracted with ethyl acetate $(4 \mathrm{x})$. The organic layers were then washed with $\mathrm{H}_{2} \mathrm{O}(2 \mathrm{x})$ and brine $(1 \mathrm{x})$, dried over sodium sulfate, filtered through cotton, and concentrated under reduced pressure. The crude material was then purified by flash chromatography ( $20 \%$ to $40 \%$ ethyl acetate:hexanes) to afford hemiacetal donor 7 (5.33 g, 50\%, $\alpha: \beta 1: 0.41)$ as a white amorphous solid.

${ }^{1} \mathrm{H}$ NMR ( $\alpha$-anomer, $\left.500 \mathrm{MHz}, \mathrm{CDCl}_{3}\right) \delta 7.85-7.77(\mathrm{~m}, 4 \mathrm{H}), 7.55(\mathrm{dd}, J=8.4,1.7 \mathrm{~Hz}, 1 \mathrm{H}), 7.50-7.42(\mathrm{~m}, 2 \mathrm{H}), 5.38$ $(\mathrm{t}, J=3.5 \mathrm{~Hz}, 1 \mathrm{H}), 5.19-5.11(\mathrm{~m}, 1 \mathrm{H}), 4.80(\mathrm{dd}, J=15.7,11.7 \mathrm{~Hz}, 1 \mathrm{H}), 4.24(\mathrm{ddd}, J=11.8,4.7,2.6 \mathrm{~Hz}, 1 \mathrm{H}), 4.08(\mathrm{q}, J$ $=6.4 \mathrm{~Hz}, 1 \mathrm{H}), 3.43(\mathrm{~s}, 1 \mathrm{H}), 2.82(\mathrm{t}, J=2.6 \mathrm{~Hz}, 1 \mathrm{H}), 2.20-2.10(\mathrm{~m}, 1 \mathrm{H}), 1.74-1.66(\mathrm{~m}, 1 \mathrm{H}), 1.15(\mathrm{~d}, J=6.4 \mathrm{~Hz}, 3 \mathrm{H})$, $0.95(\mathrm{~s}, 9 \mathrm{H}), 0.16-0.11(\mathrm{~m}, 6 \mathrm{H})$.

${ }^{13} \mathrm{C}$ NMR (126 MHz, $\left.\mathrm{CDCl}_{3}\right) \delta 136.7,136.5,133.3,133.3,133.1,128.0,127.8,127.8,127.0,126.9,126.8,126.7$, $126.1,126.0,125.9,125.8,94.7,92.8,79.0,77.7,75.2,75.0,72.0,71.1,68.6,67.0,38.2,34.0,26.1,26.0,18.2,18.2$, $17.5,17.4,-4.4,-4.4,-4.5,-4.6$.

LRMS (ESI) m/z: (M+Na)+ Calcd. $\mathrm{C}_{23} \mathrm{H}_{34} \mathrm{O}_{4} \mathrm{SiNa}$ 425.2124; Found 425.45 
$[\alpha]_{\lambda}^{24}\left(\mathrm{c}=1.25, \mathrm{CH}_{2} \mathrm{Cl}_{2}\right):-0.646$

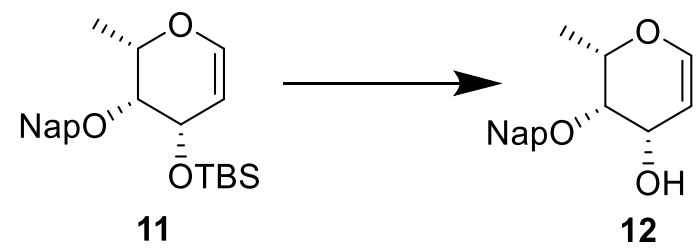

\section{4-0-(2-napthylmethyl)-L-fucal (12)}

To a solution of compound $11(1.78 \mathrm{~g}, 4.628 \mathrm{mmol})$ in THF $(1.76 \mathrm{~mL})$ was added a $1 \mathrm{M}$ solution of tetrabutylammonium fluoride in THF $(6.94 \mathrm{~mL}, 6.942 \mathrm{mmol})$ at room temperature. After 2 hours of stirring, the reaction was diluted with $\mathrm{H}_{2} \mathrm{O}$, and the aqueous phase was extracted with $\mathrm{CH}_{2} \mathrm{Cl}_{2}$. The pooled organic layers were then washed with brine $(1 \mathrm{x})$, dried over sodium sulfate, filtered through cotton, and concentrated under reduced pressure. The crude material was then purified by flash chromatography ( $10 \%$ to $30 \%$ ethyl acetate:hexanes) to afford fucal $12(1.08 \mathrm{~g}, 86 \%)$ as a white amorphous solid.

${ }^{1} \mathrm{H}$ NMR $\left(500 \mathrm{MHz}, \mathrm{CDCl}_{3}\right) \delta 7.88-7.78(\mathrm{~m}, 4 \mathrm{H}), 7.55-7.44(\mathrm{~m}, 3 \mathrm{H}), 6.36(\mathrm{dd}, J=6.1,1.7 \mathrm{~Hz}, 1 \mathrm{H}), 4.99-4.90(\mathrm{~m}$, $2 \mathrm{H}), 4.73-4.68(\mathrm{~m}, 1 \mathrm{H}), 4.44-4.36(\mathrm{~m}, 1 \mathrm{H}), 4.08(\mathrm{q}, J=6.7 \mathrm{~Hz}, 1 \mathrm{H}), 3.70(\mathrm{~d}, J=5.0 \mathrm{~Hz}, 1 \mathrm{H}), 2.21(\mathrm{~d}, J=9.8 \mathrm{~Hz}, 1 \mathrm{H})$, $1.34(\mathrm{~d}, J=6.7 \mathrm{~Hz}, 3 \mathrm{H})$.

${ }^{13} \mathrm{C}$ NMR $\left(126 \mathrm{MHz}, \mathrm{CDCl}_{3}\right) \delta 144.6,135.2,133.3,133.2,128.5,128.0,127.7,127.1,126.3,126.1,126.1,102.7$, $76.0,75.4,72.7,64.2,16.8$.

LRMS (ESI) m/z: (M+Na) ${ }^{+}$Calcd. $\mathrm{C}_{17} \mathrm{H}_{18} \mathrm{O}_{3} \mathrm{Na} 293.1154$; Found 293.36

HRMS (ESI) m/z: (M+Na) ${ }^{+}$Calcd. $\mathrm{C}_{17} \mathrm{H}_{18} \mathrm{O}_{3} \mathrm{Na} 293.1154$; Found 293.1144

$[\alpha] \lambda^{24}\left(\mathrm{c}=0.77, \mathrm{CH}_{2} \mathrm{Cl}_{2}\right): 0.106$<smiles>CCN(C)O[C@H]1[C@@H](OC)C=CO[C@@H]1C</smiles>

12

S5

\section{3-O-(methyl)-4-0-(2-napthylmethyl)-L-fucal (S5)}

To a $0{ }^{\circ} \mathrm{C}$ solution of fucal 12 (1.08 g, $4 \mathrm{mmol}$ ) in $20.2 \mathrm{~mL}$ DMF was added sodium hydride ( $60 \% \mathrm{wt}, 320 \mathrm{mg}, 8 \mathrm{mmol}$ ), and the reaction was stirred for 15 minutes. Methyl iodide $(0.5 \mathrm{~mL}, 8$ $\mathrm{mmol}$ ) was then added dropwise and the reaction was stirred from $0{ }^{\circ} \mathrm{C}$ to $\mathrm{rt}$ for 14 hours. The reaction was then cooled to $0{ }^{\circ} \mathrm{C}$ and quenched with $70 \mathrm{~mL}$ saturated ammonium chloride solution. The reaction was diluted further with $\mathrm{H}_{2} \mathrm{O}$ and the aqueous phase was extracted with 
$\mathrm{CH}_{2} \mathrm{Cl}_{2}(5 \mathrm{x})$. The organic phase was then washed with $1 \mathrm{M} \mathrm{LiCl}$ solution (4x) and brine (2x), dried over sodium sulfate, filtered through cotton, and concentrated under reduced pressure. The crude material was then purified by flash chromatography ( $0 \%$ to $4 \%$ ethyl acetate:toluene) to afford methylated fucal S5 (1.04 g, 91\%) as a clear oil.

${ }^{1} \mathrm{H}$ NMR $\left(500 \mathrm{MHz}, \mathrm{CDCl}_{3}\right) \delta 7.84-7.77(\mathrm{~m}, 4 \mathrm{H}), 7.55(\mathrm{dd}, J=8.6,1.5 \mathrm{~Hz}, 1 \mathrm{H}), 7.48-7.42(\mathrm{~m}, 2 \mathrm{H}), 6.36(\mathrm{dd}, J=6.3$, $1.7 \mathrm{~Hz}, 1 \mathrm{H}), 5.07(\mathrm{~d}, J=12.2 \mathrm{~Hz}, 1 \mathrm{H}), 4.87-4.80(\mathrm{~m}, 2 \mathrm{H}), 4.09-4.00(\mathrm{~m}, 2 \mathrm{H}), 3.75-3.70(\mathrm{~m}, 1 \mathrm{H}), 3.44(\mathrm{~s}, 3 \mathrm{H}), 1.27$ $(\mathrm{d}, J=6.6 \mathrm{~Hz}, 3 \mathrm{H})$.

${ }^{13} \mathrm{C}$ NMR $\left(126 \mathrm{MHz}, \mathrm{CDCl}_{3}\right) \delta 144.5,136.1,133.3,133.1,128.1,128.0,127.8,127.0,127.0,126.5,126.1,125.9$, 99.2, 74.6, 73.8, 72.8, 56.9, 16.6 .

LRMS (ESI) m/z: (M+Na) ${ }^{+}$Calcd. $\mathrm{C}_{18} \mathrm{H}_{20} \mathrm{O}_{3} \mathrm{Na}$ 307.1310; Found 307.18

HRMS (ESI) m/z: (M+Na) Calcd. $\mathrm{C}_{18} \mathrm{H}_{20} \mathrm{O}_{3} \mathrm{Na}$ 307.1310; Found 307.1303

$[\alpha]_{\lambda}{ }^{24}\left(\mathrm{c}=1.05, \mathrm{CH}_{2} \mathrm{Cl}_{2}\right): 0.130$

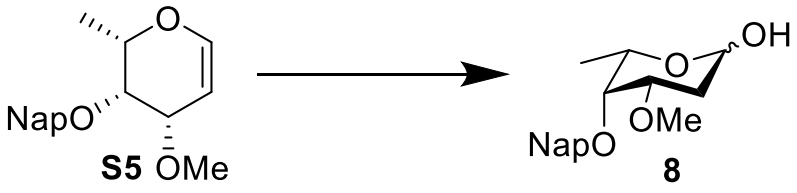

\section{2-deoxy-3-O-(methyl)-4-O-(2-napthylmethyl)- $\alpha / \beta$-L-fucopyranoside (8)}

To a solution of fucal S5 $(8.44 \mathrm{~g}, 29.684 \mathrm{mmol})$ in THF $(172 \mathrm{~mL})$ was added triphenylphosphine hydrobromide $(2.038 \mathrm{~g}, 5.937 \mathrm{mmol})$ at rt. After 10 minutes of stirring, $\mathrm{H}_{2} \mathrm{O}$ was added $(2.63 \mathrm{~mL}, 148.42 \mathrm{mmol})$ and the reaction was stirred at rt for 16.5 hours. The reaction was then quenched with saturated sodium bicarbonate solution $(250 \mathrm{~mL})$, and the aqueous phase was extracted with ethyl acetate $(6 \mathrm{x})$. The organic layers were then washed with $\mathrm{H}_{2} \mathrm{O}(3 \mathrm{x})$ and brine (1x), dried over sodium sulfate, filtered through cotton, and concentrated under reduced pressure. The crude material was then purified by flash chromatography ( $20 \%$ to $100 \%$ ethyl acetate:hexanes) to afford hemiacetal donor 8 (6.59 g, 73\%, $\alpha: \beta 1: 0.4)$ as a white amorphous solid.

${ }^{1} \mathrm{H}$ NMR ( $\alpha$-anomer, $\left.500 \mathrm{MHz}, \mathrm{CDCl}_{3}\right) \delta 7.85-7.78(\mathrm{~m}, 4 \mathrm{H}), 7.54(\mathrm{dd}, J=8.5,1.6 \mathrm{~Hz}, 1 \mathrm{H}), 7.49-7.41(\mathrm{~m}, 2 \mathrm{H}), 5.43$ $(\mathrm{t}, J=3.5 \mathrm{~Hz}, 1 \mathrm{H}), 5.05(\mathrm{~d}, J=11.8 \mathrm{~Hz}, 1 \mathrm{H}), 4.82-4.74(\mathrm{~m}, 1 \mathrm{H}), 4.04(\mathrm{q}, J=6.4 \mathrm{~Hz}, 1 \mathrm{H}), 3.73(\mathrm{ddd}, J=12.1,4.6,2.4$ $\mathrm{Hz}, 1 \mathrm{H}), 3.62(\mathrm{~s}, 1 \mathrm{H}), 3.38(\mathrm{~s}, 3 \mathrm{H}), 3.12(\mathrm{t}, J=2.6 \mathrm{~Hz}, 1 \mathrm{H}), 2.17-2.03(\mathrm{~m}, 1 \mathrm{H}), 1.93-1.87(\mathrm{~m}, 1 \mathrm{H}), 1.16(\mathrm{~d}, J=6.5 \mathrm{~Hz}$, $3 \mathrm{H})$.

${ }^{13} \mathrm{C} \mathrm{NMR}\left(126 \mathrm{MHz}, \mathrm{CDCl}_{3}\right) \delta 136.4,136.3,133.3,133.1,128.0,128.0,127.8,127.1,127.1,127.0,126.8,126.7$, 126.7, 126.1, 126.0, 125.9, 125.9, 94.9, 92.7, 80.2, 76.7, 75.2, 74.6, 74.4, 74.1, 71.2, 67.0, 56.4, 56.3, 34.2, 30.3, $17.6,17.4$.

LRMS (ESI) m/z: (M+Na) ${ }^{+}$Calcd. $\mathrm{C}_{18} \mathrm{H}_{22} \mathrm{O}_{4} \mathrm{Na} 325.1416$; Found 325.27

HRMS (ESI) m/z: (M+Na) ${ }^{+}$Calcd. $\mathrm{C}_{18} \mathrm{H}_{22} \mathrm{O}_{4} \mathrm{Na}$ 325.1416; Found 325.1407 
$[\alpha]_{\lambda}{ }^{24}\left(\mathrm{c}=5.0, \mathrm{CH}_{2} \mathrm{Cl}_{2}\right):-0.540$

S2.5 Synthesis of monosaccharide acceptor 6
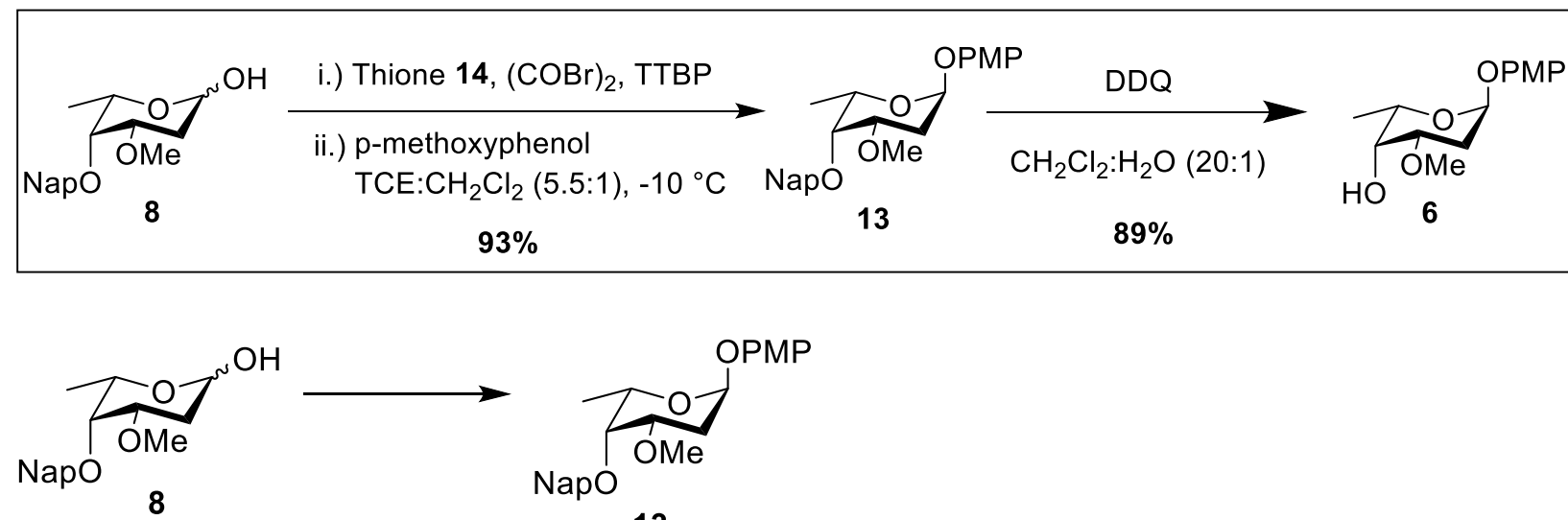

13

p-methoxyphenyl 2-deoxy-3-0-(methyl)-4-0-(2-napthylmethyl)- $\alpha$-L-fucopyranoside (13)

A solution of 2,3-bis(2,3,4-trimethoxyphenyl)cycloprop-2-ene-1-thione (14, $8.78 \mathrm{~g}, 21.83$ $\mathrm{mmol})$ in TCE $(75 \mathrm{~mL})$ and $\mathrm{CH}_{2} \mathrm{Cl}_{2}(3.7 \mathrm{~mL})$ was treated dropwise with a solution of oxalyl bromide in $\mathrm{CH}_{2} \mathrm{Cl}_{2}(2.0 \mathrm{M}, 10.91 \mathrm{~mL}, 21.83 \mathrm{mmol})$ at room temperature. After 5 minutes of stirring, the reaction was cooled to $-10{ }^{\circ} \mathrm{C}$ and a solution of p-methoxyphenol acceptor $(3.69 \mathrm{~g}, 29.77 \mathrm{mmol})$ in $\mathrm{CH}_{2} \mathrm{Cl}_{2}(7 \mathrm{~mL})$ was added slowly. A solution of hemiacetal donor 8 (3 g, $\left.9.92 \mathrm{mmol}\right)$ and TTBP proton scavenger $(9.14 \mathrm{~g}, 36.69 \mathrm{mmol})$ in TCE $(60 \mathrm{~mL})$ and $\mathrm{CH}_{2} \mathrm{Cl}_{2}(3 \mathrm{~mL})$ was then added via cannula to the reaction over a period of 10 minutes. The reaction was stirred at $-10{ }^{\circ} \mathrm{C}$ for 30 minutes and quenched with $\mathrm{Et}_{3} \mathrm{~N}(3.05 \mathrm{~mL}, 21.83 \mathrm{mmol})$ upon consumption of compound 8 by TLC. The reaction was then diluted with $\mathrm{CH}_{2} \mathrm{Cl}_{2}$, and the organic layer was washed with saturated sodium bicarbonate (2x), brine (1x), dried over $\mathrm{Na}_{2} \mathrm{SO}_{4}$, filtered through cotton, and concentrated under reduced pressure. The crude material was then purified by flash chromatography ( $2 \%$ to $10 \%$ acetone:hexanes) and dried on high vacuum to afford compound 13 (3.77 g, 93\%, single $\alpha$ anomer) as a beige oil.

${ }^{1} \mathrm{H}$ NMR $\left(500 \mathrm{MHz}, \mathrm{CDCl}_{3}\right) \delta 7.86-7.79(\mathrm{~m}, 4 \mathrm{H}), 7.57(\mathrm{dd}, J=8.4,1.7 \mathrm{~Hz}, 1 \mathrm{H}), 7.51-7.42(\mathrm{~m}, 2 \mathrm{H}), 7.02-6.95(\mathrm{~m}$, $2 \mathrm{H}), 6.84-6.77(\mathrm{~m}, 2 \mathrm{H}), 5.59(\mathrm{~d}, J=2.4 \mathrm{~Hz}, 1 \mathrm{H}), 5.10(\mathrm{~d}, J=11.9 \mathrm{~Hz}, 1 \mathrm{H}), 4.83(\mathrm{~d}, J=11.9 \mathrm{~Hz}, 1 \mathrm{H}), 3.95(\mathrm{~d}, J=6.6$ $\mathrm{Hz}, 1 \mathrm{H}), 3.88$ (ddd, $J=12.1,4.6,2.4 \mathrm{~Hz}, 1 \mathrm{H}), 3.76(\mathrm{~s}, 3 \mathrm{H}), 3.70(\mathrm{~s}, 1 \mathrm{H}), 3.47(\mathrm{~s}, 3 \mathrm{H}), 2.28(\mathrm{td}, J=12.4,3.7 \mathrm{~Hz}, 1 \mathrm{H})$, $2.15(\mathrm{dd}, J=12.7,4.7 \mathrm{~Hz}, 1 \mathrm{H}), 1.16(\mathrm{~d}, J=6.5 \mathrm{~Hz}, 3 \mathrm{H})$.

${ }^{13} \mathrm{C}$ NMR $\left(126 \mathrm{MHz} \mathrm{CDCl}_{3}\right) \delta 154.6,151.1,136.3,133.2,133.0,128.0,127.9,127.7,126.9,126.6,126.0,125.8$, 117.6, 114.5, 97.3, 76.9, 74.9, 74.5, 67.5, 56.3, 55.7, 30.5, 17.4.

LRMS (ESI) m/z: (M+Na) ${ }^{+}$Calcd. $\mathrm{C}_{25} \mathrm{H}_{28} \mathrm{O}_{5} \mathrm{Na} 431.1834$; Found 431.1937

HRMS (ESI) m/z: $(\mathrm{M}+\mathrm{Na})^{+}$Calcd. $\mathrm{C}_{25} \mathrm{H}_{28} \mathrm{O}_{5} \mathrm{Na}$ 431.1834; Found 431.1819

$[\alpha]_{\lambda}{ }^{24}\left(\mathrm{c}=2.3, \mathrm{CH}_{2} \mathrm{Cl}_{2}\right):-0.583$ 


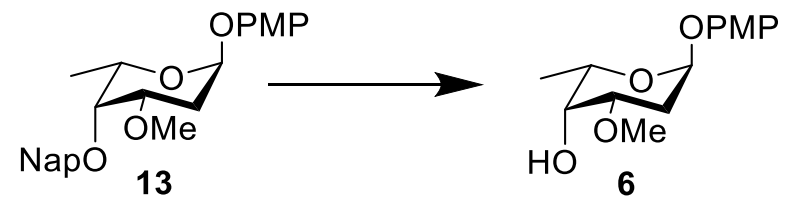

p-methoxyphenyl 2-deoxy-3-O-(methyl)- $\alpha$-L-fucopyranoside (6)

To a solution of compound 13 (3.77 g, $9.23 \mathrm{mmol})$ in $\mathrm{CH}_{2} \mathrm{Cl}_{2}(476 \mathrm{~mL})$ and $\mathrm{H}_{2} \mathrm{O}(23.8 \mathrm{~mL})$ was added solid DDQ (4.19 $\mathrm{g}, 18.46 \mathrm{mmol})$. The reaction was stirred at room temperature for 80 minutes, and then diluted with $\mathrm{CH}_{2} \mathrm{Cl}_{2}$ and $2 \mathrm{M}$ sodium hydroxide solution. The organic layer was washed with $2 \mathrm{M}$ sodium hydroxide solution $(2 \mathrm{x})$, and the pooled aqueous layers were backextracted with $\mathrm{CH}_{2} \mathrm{Cl}_{2}(2 \mathrm{x})$. The pooled organic layers were then dried over sodium sulfate, filtered through cotton, and concentrated under reduced pressure. The crude material was then purified by automated flash chromatography (36\% to $57 \%$ ethyl acetate:hexanes) and dried on high vacuum to afford monosaccharide acceptor $6(2.21 \mathrm{~g}, 89 \%)$ as a white amorphous solid.

${ }^{1} \mathrm{H}$ NMR $\left(500 \mathrm{MHz}, \mathrm{CDCl}_{3}\right) \delta 7.03-6.96(\mathrm{~m}, 2 \mathrm{H}), 6.86-6.79(\mathrm{~m}, 2 \mathrm{H}), 5.54(\mathrm{~d}, J=2.6 \mathrm{~Hz}, 1 \mathrm{H}), 4.00(\mathrm{q}, J=6.6 \mathrm{~Hz}, 1 \mathrm{H})$, $3.87-3.80(\mathrm{~m}, 2 \mathrm{H}), 3.77(\mathrm{~s}, 3 \mathrm{H}), 3.45(\mathrm{~s}, 3 \mathrm{H}), 2.17(\mathrm{~s}, 1 \mathrm{H}), 2.14-2.06(\mathrm{~m}, 1 \mathrm{H}), 2.04-1.95(\mathrm{~m}, 1 \mathrm{H}), 1.28(\mathrm{~d}, J=6.6 \mathrm{~Hz}$, $3 \mathrm{H})$.

${ }^{13} \mathrm{C} \mathrm{NMR}\left(126 \mathrm{MHz}, \mathrm{CDCl}_{3}\right) \delta 154.7,151.0,117.6,114.6,96.9,74.6,67.6,66.3,55.7,55.7,29.7,16.8$.

LRMS (ESI) m/z: (M+Na) ${ }^{+}$Calcd. $\mathrm{C}_{14} \mathrm{H}_{20} \mathrm{O}_{5} \mathrm{Na} 291.1208$; Found 291.27

HRMS (ESI) m/z: (M+Na) ${ }^{+}$Calcd. $\mathrm{C}_{14} \mathrm{H}_{20} \mathrm{O}_{5} \mathrm{Na} 291.1208$; Found 291.1200

$[\alpha]_{\lambda}{ }^{24}\left(\mathrm{c}=2.3, \mathrm{CH}_{2} \mathrm{Cl}_{2}\right):-0.496$

S2.6 Synthesis of disaccharides 15, 16

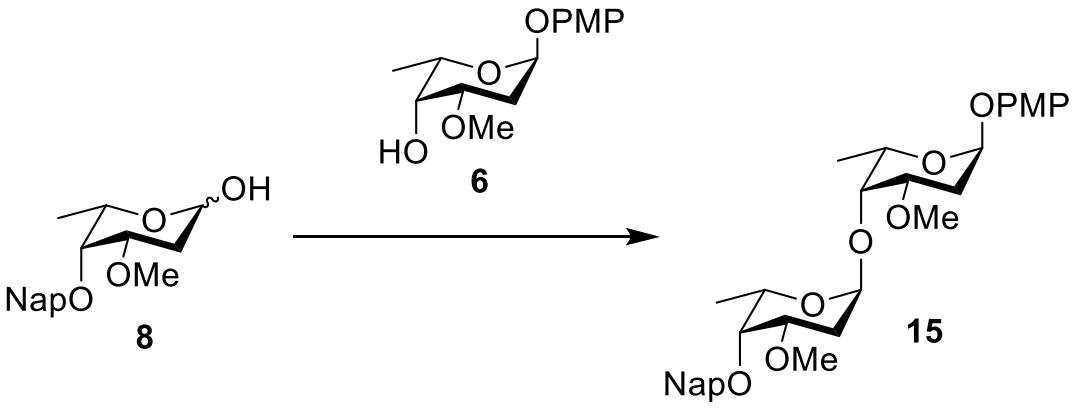

p-methoxyphenyl 2-deoxy-3-0-(methyl)-4-0-(2-deoxy-3-0-(methyl)-4-0-(2-napthylmethyl)- $\alpha$ L-fucopyranosyl)- $\alpha$-L-fucopyranoside (15) 
A solution of 2,3-bis(2,3,4-trimethoxyphenyl)cycloprop-2-ene-1-thione (14, $879 \mathrm{mg}, 2.18$ $\mathrm{mmol})$ in TCE $(8.15 \mathrm{~mL})$ and $\mathrm{CH}_{2} \mathrm{Cl}_{2}(5 \mathrm{~mL})$ was treated dropwise with a solution of oxalyl bromide in $\mathrm{CH}_{2} \mathrm{Cl}_{2}(2 \mathrm{M}, 1.09 \mathrm{~mL}, 2.183 \mathrm{mmol})$ and stirred at room temperature for 5 minutes. The reaction was then cooled to $-10{ }^{\circ} \mathrm{C}$, and a $-10{ }^{\circ} \mathrm{C}$ solution of hemiacetal donor $8(300 \mathrm{mg}, 0.99 \mathrm{mmol})$ and TTBP $(1.48 \mathrm{~g}, 5.95 \mathrm{mmol})$ in TCE $(20 \mathrm{~mL})$ and $\mathrm{CH}_{2} \mathrm{Cl}_{2}(4 \mathrm{~mL})$ was added dropwise via cannula over a period of 15 minutes. After stirring an additional 5 minutes at $-10{ }^{\circ} \mathrm{C}$, the reaction was treated dropwise with a solution of acceptor 6 in $\mathrm{CH}_{2} \mathrm{Cl}_{2}(9 \mathrm{~mL})$. The reaction was stirred for 90 minutes until consumption of the donor was observed by TLC, and was quenched at $-10{ }^{\circ} \mathrm{C}$ via dropwise addition of $\mathrm{Et}_{3} \mathrm{~N}(0.69 \mathrm{~mL}, 4.94 \mathrm{mmol})$. The reaction was then diluted with $\mathrm{CH}_{2} \mathrm{Cl}_{2}$, washed with saturated sodium bicarbonate $(2 x)$, and the pooled aqueous layers were back-extracted with $\mathrm{CH}$ ${ }_{2} \mathrm{Cl}_{2}(2 \mathrm{x})$. The pooled organic layers were washed with brine (1x), dried over sodium sulfate, filtered through cotton, and concentrated under reduced pressure. The crude material was purified by automated flash chromatography (10\% to $30 \%$ ethyl acetate:hexanes) and dried on high vacuum to afford disaccharide 15 (380 mg, 69\%, $\alpha$-anomer only) as a beige amorphous solid.

${ }^{1} \mathrm{H}$ NMR $\left(500 \mathrm{MHz}, \mathrm{CDCl}_{3}\right) \delta 7.84-7.78(\mathrm{~m}, 4 \mathrm{H}), 7.55(\mathrm{~d}, J=8.4 \mathrm{~Hz}, 1 \mathrm{H}), 7.48-7.40(\mathrm{~m}, 2 \mathrm{H}), 6.99(\mathrm{~d}, J=8.6 \mathrm{~Hz}, 2 \mathrm{H})$, $6.81(\mathrm{~d}, J=8.6 \mathrm{~Hz}, 2 \mathrm{H}), 5.57(\mathrm{~s}, 1 \mathrm{H}), 5.12-5.02(\mathrm{~m}, 2 \mathrm{H}), 4.79(\mathrm{~d}, J=11.9 \mathrm{~Hz}, 1 \mathrm{H}), 4.29(\mathrm{q}, J=6.4 \mathrm{~Hz}, 1 \mathrm{H}), 3.94(\mathrm{q}, J=$ $6.5 \mathrm{~Hz}, 1 \mathrm{H}), 3.88(\mathrm{~s}, 1 \mathrm{H}), 3.83-3.70(\mathrm{~m}, 5 \mathrm{H}), 3.66(\mathrm{~s}, 1 \mathrm{H}), 3.44(\mathrm{~s}, 3 \mathrm{H}), 3.42(\mathrm{~s}, 3 \mathrm{H}), 2.22-2.06(\mathrm{~m}, 3 \mathrm{H}), 2.05-1.97$ $(\mathrm{m}, 1 \mathrm{H}), 1.23-1.17(\mathrm{~m}, 6 \mathrm{H})$.

${ }^{13} \mathrm{C}$ NMR $\left(126 \mathrm{MHz}, \mathrm{CDCl}_{3}\right) \delta 154.7,151.1,136.6,133.3,133.0,127.9,127.9,127.7,126.7,126.5,125.9,125.7$, 117.6, 114.6, 99.7, 97.2, 76.9, 75.2, 75.0, 74.4, 73.6, 67.7, 67.1, 56.5, 56.1, 55.7, 31.1, 30.6, 17.6, 17.5.

LRMS (ESI) m/z: (M+Na) ${ }^{+}$Calcd. $\mathrm{C}_{32} \mathrm{H}_{40} \mathrm{O}_{8} \mathrm{Na}$ 575.2621; Found 575.36

HRMS (ESI) m/z: $(\mathrm{M}+\mathrm{Na})^{+}$Calcd. $\mathrm{C}_{32} \mathrm{H}_{40} \mathrm{O}_{8} \mathrm{Na} 575.2621$; Found 575.2614

$[\alpha]_{\lambda}{ }^{24}\left(\mathrm{c}=0.1, \mathrm{CH}_{2} \mathrm{Cl}_{2}\right):-1.300$

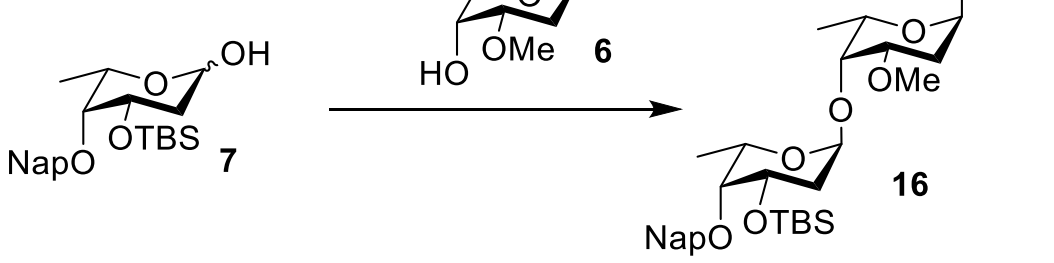

p-methoxyphenyl 2-deoxy-3-0-(methyl)-4-0-(2-deoxy-3-0-(tert-butyldimethylsilyl)-4-0-(2napthylmethyl)- $\alpha$-L-fucopyranosyl)- $\alpha$-L-fucopyranoside (16)

A solution of 2,3-bis(2,3,4-trimethoxyphenyl)cycloprop-2-ene-1-thione (14, $2.20 \mathrm{~g}, 5.46$ $\mathrm{mmol})$ in TCE $(21.7 \mathrm{~mL})$ and $\mathrm{CH}_{2} \mathrm{Cl}_{2}(8.57 \mathrm{~mL})$ was treated dropwise with a solution of oxalyl bromide in $\mathrm{CH}_{2} \mathrm{Cl}_{2}(2 \mathrm{M}, 2.73 \mathrm{~mL}, 5.46 \mathrm{mmol})$ and stirred at room temperature for 5 minutes. The reaction was then cooled to $-10{ }^{\circ} \mathrm{C}$, and a $0{ }^{\circ} \mathrm{C}$ solution of hemiacetal donor 7 (1.00 g, $\left.2.48 \mathrm{mmol}\right)$ and TTBP $(3.70 \mathrm{~g}, 14.88 \mathrm{mmol})$ in TCE $(50 \mathrm{~mL})$ and $\mathrm{CH}_{2} \mathrm{Cl}_{2}(10 \mathrm{~mL})$ was added dropwise via cannula over a period of 30 minutes. After stirring an additional 5 minutes at $-10^{\circ} \mathrm{C}$, the reaction was 
treated dropwise with a solution of acceptor 6 in $\mathrm{CH}_{2} \mathrm{Cl}_{2}(25 \mathrm{~mL})$. The reaction was stirred for 3.5 hours until consumption of the donor was observed by TLC and was quenched at $-10{ }^{\circ} \mathrm{C}$ via dropwise addition of $\mathrm{Et}_{3} \mathrm{~N}(1.74 \mathrm{~mL}, 12.46 \mathrm{mmol})$. The reaction was then diluted with $\mathrm{CH}_{2} \mathrm{Cl}_{2}$, washed with saturated sodium bicarbonate $(2 x)$, and the pooled aqueous layers were backextracted with $\mathrm{CH}_{2} \mathrm{Cl}_{2}(3 \mathrm{x})$. The pooled organic layers were washed with brine $(1 \mathrm{x})$, dried over sodium sulfate, filtered through cotton, and concentrated under reduced pressure. The crude material was purified by flash chromatography (5\% to $20 \%$ ethyl acetate:hexanes) and dried on high vacuum to afford disaccharide 16 as a white amorphous solid as a mixture with free $p$ methoxyphenol [1:0.26] by ${ }^{1} \mathrm{H}-\mathrm{NMR}$ integration (1.06 g (16), 65\%, $\alpha$-anomer only). Preparatory thin layer chromatography $(15 \%$ ethyl acetate:hexanes) yielded the $\alpha$-anomer for characterization below.

${ }^{1} \mathrm{H}$ NMR $\left(500 \mathrm{MHz}, \mathrm{CDCl}_{3}\right) \delta 7.90-7.74(\mathrm{~m}, 4 \mathrm{H}), 7.55(\mathrm{~d}, J=8.7 \mathrm{~Hz}, 1 \mathrm{H}), 7.49-7.41(\mathrm{~m}, 2 \mathrm{H}), 6.99(\mathrm{~d}, J=8.6 \mathrm{~Hz}, 2 \mathrm{H})$, $6.82(\mathrm{~d}, J=8.6 \mathrm{~Hz}, 2 \mathrm{H}), 5.54(\mathrm{~s}, 1 \mathrm{H}), 5.17(\mathrm{~d}, J=11.8 \mathrm{~Hz}, 1 \mathrm{H}), 5.03(\mathrm{~s}, 1 \mathrm{H}), 4.81(\mathrm{~d}, J=11.9 \mathrm{~Hz}, 1 \mathrm{H}), 4.35-4.26(\mathrm{~m}$, $2 \mathrm{H}), 3.93(\mathrm{q}, J=6.7 \mathrm{~Hz}, 1 \mathrm{H}), 3.89(\mathrm{~s}, 1 \mathrm{H}), 3.81-3.73(\mathrm{~m}, 4 \mathrm{H}), 3.47-3.41(\mathrm{~m}, 4 \mathrm{H}), 2.24(\mathrm{td}, J=12.2,3.6 \mathrm{~Hz}, 1 \mathrm{H}), 2.08$ (td, $J=12.3,3.7 \mathrm{~Hz}, 1 \mathrm{H}), 1.99(\mathrm{dd}, J=12.5,4.6 \mathrm{~Hz}, 1 \mathrm{H}), 1.90(\mathrm{dd}, J=12.6,4.5 \mathrm{~Hz}, 1 \mathrm{H}), 1.18(\mathrm{~d}, J=6.5 \mathrm{~Hz}, 6 \mathrm{H}), 0.95$ $(\mathrm{s}, 9 \mathrm{H}), 0.14(\mathrm{~d}, J=7.9 \mathrm{~Hz}, 6 \mathrm{H})$.

${ }^{13} \mathrm{C}$ NMR $\left(126 \mathrm{MHz}, \mathrm{CDCl}_{3}\right) \delta 154.7,151.0,136.9,133.3,132.9,127.9,127.8,127.6,126.6,126.5,125.8,125.6$, $117.8,114.5,99.4,97.4,79.3,75.0,74.8,72.9,69.0,67.7,67.1,56.4,55.7,33.9,31.2,25.9,18.2,17.5,17.4,-4.5,-$ 4.6.

LRMS (ESI) m/z: (M+Na)+ Calcd. $\mathrm{C}_{37} \mathrm{H}_{52} \mathrm{O}_{8} \mathrm{SiNa}$ 675.3329; Found 673.18

HRMS (ESI) m/z: (M+Na) ${ }^{+}$Calcd. $\mathrm{C}_{37} \mathrm{H}_{52} \mathrm{O}_{8} \mathrm{SiNa}$ 675.3329; Found 675.3327

$[\alpha]_{\lambda}^{24}\left(c=1.75, \mathrm{CH}_{2} \mathrm{Cl}_{2}\right):-0.470$

\section{S2.7 Disaccharide deprotections}

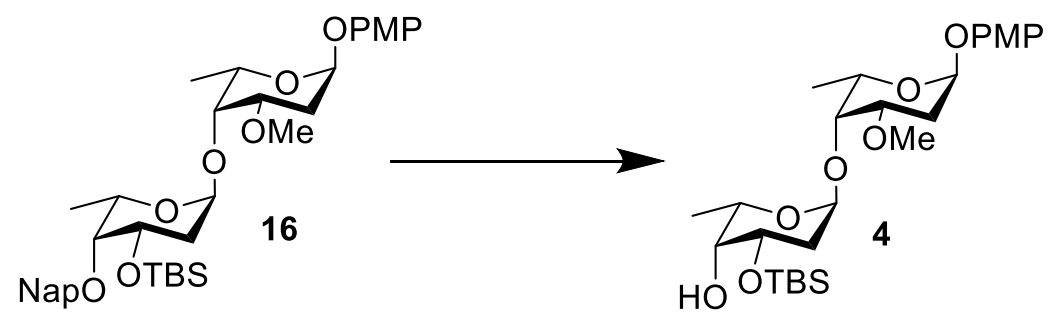

\section{p-methoxyphenyl 2-deoxy-3-0-(methyl)-4-0-(2-deoxy-3-0-(tert-butyldimethylsilyl)- $\alpha-L-$} fucopyranosyl)- $\alpha$-L-fucopyranoside (4)

To a solution of compound 16 (325 mg, $0.50 \mathrm{mmol})$ in $\mathrm{CH}_{2} \mathrm{Cl}_{2}(25.64 \mathrm{~mL})$ and $\mathrm{H}_{2} \mathrm{O}(1.28$ $\mathrm{mL}$ ) was added solid DDQ $(226 \mathrm{mg}, 1.00 \mathrm{mmol})$. The reaction was stirred at room temperature for 2.8 hours, and then diluted with $\mathrm{CH}_{2} \mathrm{Cl}_{2}$, washed with $2 \mathrm{M}$ sodium hydroxide solution ( $3 \mathrm{x}$ ), and the pooled aqueous layers were back-extracted with $\mathrm{CH}_{2} \mathrm{Cl}_{2}(2 \mathrm{x})$. The pooled organic layers were then washed with brine, dried over sodium sulfate, filtered through cotton, and concentrated under reduced pressure. The crude material was then purified by automated flash 
chromatography (19\% to $40 \%$ ethyl acetate:hexanes) and dried on high vacuum to afford disaccharide acceptor 4 (228 $\mathrm{mg}, 89 \%)$ as a yellow oil.

${ }^{1} \mathrm{H}$ NMR $\left(500 \mathrm{MHz}, \mathrm{CDCl}_{3}\right) \delta 7.03-6.96(\mathrm{~m}, 2 \mathrm{H}), 6.85-6.78(\mathrm{~m}, 2 \mathrm{H}), 5.56(\mathrm{~s}, 1 \mathrm{H}), 4.99(\mathrm{~s}, 1 \mathrm{H}), 4.36(\mathrm{q}, J=6.7 \mathrm{~Hz}$, $1 \mathrm{H}), 4.15$ (ddd, $J=11.0,5.6,3.2 \mathrm{~Hz}, 1 \mathrm{H}), 3.95(\mathrm{q}, J=6.4 \mathrm{~Hz}, 1 \mathrm{H}), 3.90(\mathrm{~s}, 1 \mathrm{H}), 3.77(\mathrm{~s}, 4 \mathrm{H}), 3.54(\mathrm{~s}, 1 \mathrm{H}), 3.48(\mathrm{~s}, 3 \mathrm{H})$, $2.35(\mathrm{~s}, 1 \mathrm{H}), 2.09(\mathrm{td}, J=12.3,3.7 \mathrm{~Hz}, 1 \mathrm{H}), 2.01(\mathrm{dd}, J=12.5,4.6 \mathrm{~Hz}, 1 \mathrm{H}), 1.97-1.84(\mathrm{~m}, 2 \mathrm{H}), 1.30(\mathrm{~d}, J=6.7 \mathrm{~Hz}$, $3 \mathrm{H}), 1.19(\mathrm{~d}, J=6.6 \mathrm{~Hz}, 3 \mathrm{H}), 0.90(\mathrm{~s}, 9 \mathrm{H}), 0.10(\mathrm{~s}, 6 \mathrm{H})$.

${ }^{13} \mathrm{C} \mathrm{NMR}\left(126 \mathrm{MHz}, \mathrm{CDCl}_{3}\right) \delta 154.8,151.2,117.8,114.7,99.4,97.4,75.1,73.6,71.4,67.8,66.9,65.7,56.6,55.8$, $32.9,31.2,25.9,18.1,17.6,17.0,-4.4,-4.5$.

LRMS (ESI) m/z: (M+Na) ${ }^{+}$Calcd. $\mathrm{C}_{26} \mathrm{H}_{44} \mathrm{O}_{8} \mathrm{SiNa} 535.2703$; Found 535.36

HRMS (ESI) m/z: (M+Na) ${ }^{+}$Calcd. $\mathrm{C}_{26} \mathrm{H}_{44} \mathrm{O}_{8} \mathrm{SiNa} 535.2703$; Found 535.2697

$[\alpha]_{\lambda}{ }^{24}\left(\mathrm{c}=1.4, \mathrm{CH}_{2} \mathrm{Cl}_{2}\right):-1.283$

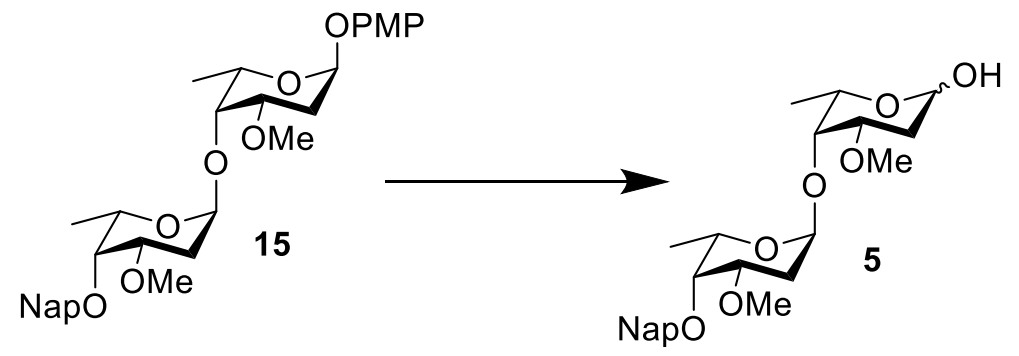

\section{2-deoxy-3-0-(methyl)-4-0-(2-deoxy-3-0-(methyl)-4-0-(2-napthylmethyl)- $\alpha$-L-fucopyranosyl)- $\alpha /$-L-fucopyranoside (5)}

To a suspension of disaccharide $15(380 \mathrm{mg}, 0.69 \mathrm{mmol})$ and solid sodium bicarbonate (116 mg, $1.38 \mathrm{mmol}$ ) in acetonitrile $(36.42 \mathrm{~mL})$ and $\mathrm{H}_{2} \mathrm{O}(4.05 \mathrm{~mL})$ was added ceric ammonium nitrate $(754 \mathrm{mg}, 1.38 \mathrm{mmol}$ ) at room temperature. The reaction was quenched after 45 minutes of stirring with saturated sodium bicarbonate solution and diluted with $\mathrm{H}_{2} \mathrm{O}$ and $\mathrm{CH}_{2} \mathrm{Cl}_{2}$. The aqueous layer was extracted with $\mathrm{CH}_{2} \mathrm{Cl}_{2}(3 \mathrm{x})$, and the pooled organic layers were then washed with brine $(1 \mathrm{x})$, dried over sodium sulfate, filtered through cotton, and concentrated under reduced pressure. The crude material was then purified by automated flash chromatography ( $36 \%$ to $87 \%$ ethyl acetate: $\mathrm{CH}_{2} \mathrm{Cl}_{2}$ ) and dried on high vacuum to afford disaccharide hemiacetal donor 5 (198 $\mathrm{mg}, 64 \%, \alpha: \beta=1: 0.43$ ) as a beige amorphous solid.

${ }^{1} \mathrm{H}$ NMR ( $\alpha$-anomer, $\left.500 \mathrm{MHz}, \mathrm{CDCl}_{3}\right) \delta 7.86-7.77(\mathrm{~m}, 4 \mathrm{H}), 7.55(\mathrm{dd}, J=8.4,1.7 \mathrm{~Hz}, 1 \mathrm{H}), 7.50-7.41(\mathrm{~m}, 2 \mathrm{H}), 5.43$ $(\mathrm{s}, 1 \mathrm{H}), 5.08(\mathrm{~d}, J=11.6 \mathrm{~Hz}, 1 \mathrm{H}), 5.03(\mathrm{~d}, J=3.9 \mathrm{~Hz}, 1 \mathrm{H}), 4.79(\mathrm{~d}, J=12.0 \mathrm{~Hz}, 1 \mathrm{H}), 4.30-4.22(\mathrm{~m}, 1 \mathrm{H}), 4.05(\mathrm{q}, J=6.5$ $\mathrm{Hz}, 1 \mathrm{H}), 3.84(\mathrm{~d}, J=2.7 \mathrm{~Hz}, 1 \mathrm{H}), 3.79-3.72(\mathrm{~m}, 2 \mathrm{H}), 3.69-3.62(\mathrm{~m}, 2 \mathrm{H}), 3.41(\mathrm{~s}, 3 \mathrm{H}), 3.40(\mathrm{~s}, 3 \mathrm{H}), 2.38(\mathrm{t}, J=2.4 \mathrm{~Hz}$, $1 \mathrm{H}), 2.21-2.07(\mathrm{~m}, 2 \mathrm{H}), 1.96(\mathrm{tdd}, J=12.4,3.6,2.1 \mathrm{~Hz}, 1 \mathrm{H}), 1.84(\mathrm{dd}, J=12.5,4.6 \mathrm{~Hz}, 1 \mathrm{H}) 1.22(\mathrm{~d}, J=6.6 \mathrm{~Hz}, 3 \mathrm{H})$, $1.20-1.16(\mathrm{~m}, 3 \mathrm{H})$.

${ }^{13} \mathrm{C} \mathrm{NMR}\left(126 \mathrm{MHz}, \mathrm{CDCl}_{3}\right) \delta 136.7,133.4,133.1,128.0,128.0,127.8,126.9,126.8,126.7,126.6,126.0,125.8$, 100.0, 99.7, 94.7, 92.7, 78.5, 77.0, 75.3, 74.7, 74.5, 73.9, 72.8, 71.4, 67.2, 67.2, 67.1, 56.9, 56.6, 56.3, 56.3, 34.4, $31.0,30.7,30.6,17.7,17.7,17.6$. 
LRMS (ESI) m/z: (M+Na) ${ }^{+}$Calcd. $\mathrm{C}_{25} \mathrm{H}_{34} \mathrm{O} 7 \mathrm{Na}$ 469.2202; Found 469.36

HRMS (ESI) m/z: (M+Na) $)^{+}$Calcd. $\mathrm{C}_{25} \mathrm{H}_{34} \mathrm{O}_{7} \mathrm{Na}$ 469.2202; Found 469.2192

$[\alpha]_{\lambda}^{24}\left(\mathrm{c}=1.3, \mathrm{CH}_{2} \mathrm{Cl}_{2}\right):-0.262$

S2.8 Synthesis of tetrasaccharide 3 and fumarate 2

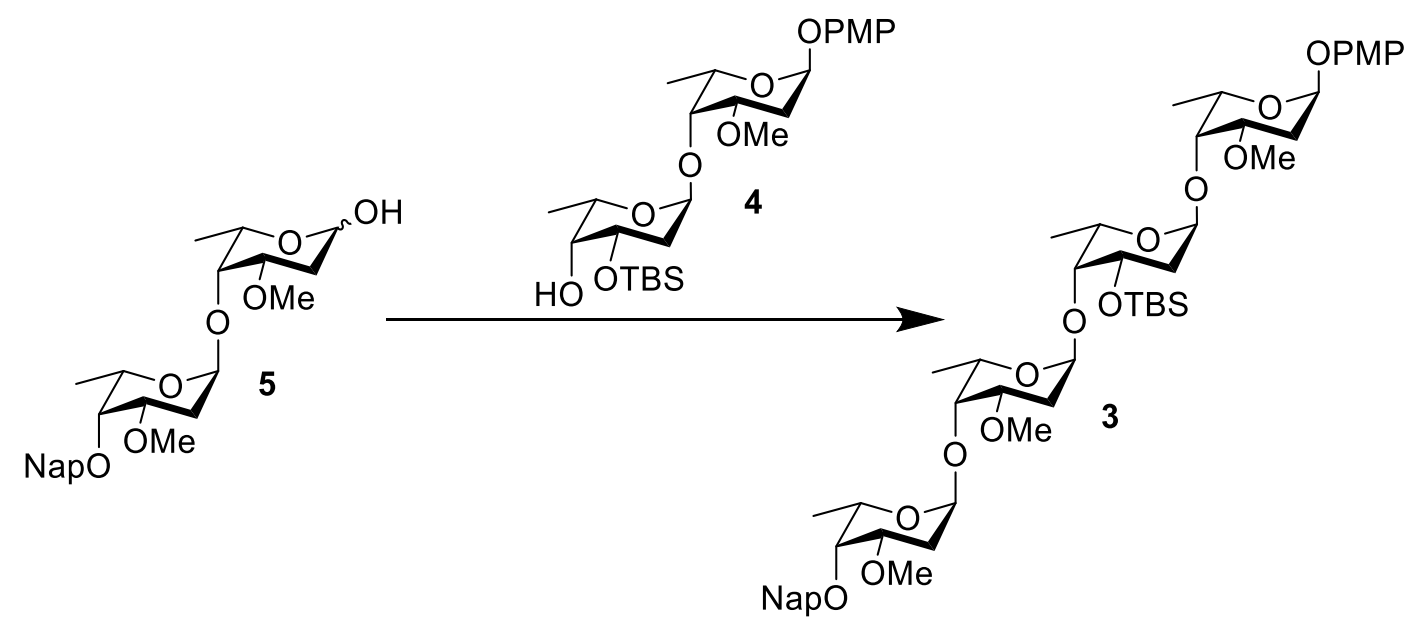

p-methoxyphenyl 2-deoxy-3-0-(methyl)-4-0-(2-deoxy-3-0-(tert-butyldimethylsilyl)-4-0-(2deoxy-3-0-methyl-4-0-(2-deoxy-3-0-methyl-4-0-(2-napthylmethyl)- $\alpha$-L-fucopyranosyl)- $\alpha-L-$ fucopyranosyl)- $\alpha$-L-fucopyranosyl)- $\alpha$-L-fucopyranoside (3)

A solution of 2,3-bis(2,3,4-trimethoxyphenyl)cycloprop-2-ene-1-thione (14, $159 \mathrm{mg}, 0.39$ $\mathrm{mmol})$ in TCE $(1.56 \mathrm{~mL})$ and $\mathrm{CH}_{2} \mathrm{Cl}_{2}(0.77 \mathrm{~mL})$ was treated dropwise with a solution of oxalyl bromide in $\mathrm{CH}_{2} \mathrm{Cl}_{2}(2 \mathrm{M}, 0.200 \mathrm{~mL}, 0.39 \mathrm{mmol})$ and stirred at room temperature for 5 minutes. The reaction was then cooled to $-30{ }^{\circ} \mathrm{C}$, and a $0{ }^{\circ} \mathrm{C}$ solution of disaccharide donor $5(80 \mathrm{mg}, 0.18$ $\mathrm{mmol}$ ) and TTBP (267 mg, $1.07 \mathrm{mmol})$ in TCE $(3.5 \mathrm{~mL})$ and $\mathrm{CH}_{2} \mathrm{Cl}_{2}(0.75 \mathrm{~mL})$ was added dropwise over a period of 10 minutes. After stirring an additional 5 minutes at $-30{ }^{\circ} \mathrm{C}$, the reaction was treated dropwise with a solution of disaccharide acceptor $4(275 \mathrm{mg}, 0.54 \mathrm{mmol})$ in $\mathrm{CH}_{2} \mathrm{Cl}_{2}(0.75$ $\mathrm{mL}$ ). The reaction was then allowed to warm to $-10{ }^{\circ} \mathrm{C}$ over a period of 35 minutes and was then stirred at $-10^{\circ} \mathrm{C}$ for 3.25 hours until consumption of the disaccharide donor 5 was evident by TLC. The reaction was then quenched at $-10{ }^{\circ} \mathrm{C}$ via dropwise addition of $\mathrm{Et}_{3} \mathrm{~N}(0.05 \mathrm{~mL}, 0.74$ mmol) and diluted with $\mathrm{CH}_{2} \mathrm{Cl}_{2}$. The organic phase was washed with saturated sodium bicarbonate solution (2x), and the pooled aqueous layers were back-extracted with $\mathrm{CH}_{2} \mathrm{Cl}_{2}(2 \mathrm{x})$. The pooled organic layers were then washed with brine (1x), dried over sodium sulfate, filtered through cotton, and concentrated under reduced pressure. The crude material was purified first by manual flash chromatography ( $10 \%$ to $50 \%$ ethyl acetate:toluene), then by automated flash chromatography (30\% to 54\% ethyl acetate:hexanes), and then dried on high vacuum to afford tetrasaccharide 3 (52 mg, 31\%, $\alpha$-anomer only) as a white amorphous solid. 
$\mathrm{Hz}, 1 \mathrm{H}), 4.72(\mathrm{~d}, J=12.0 \mathrm{~Hz}, 1 \mathrm{H}), 4.52-4.37(\mathrm{~m}, 4 \mathrm{H}), 3.86$ (dddd, J=12.4, 10.5, 4.4, $2.5 \mathrm{~Hz}, 2 \mathrm{H}), 3.81(\mathrm{~s}, 1 \mathrm{H}), 3.75$ $(\mathrm{s}, 1 \mathrm{H}), 3.73-3.66(\mathrm{~m}, 2 \mathrm{H}), 3.54(\mathrm{~s}, 1 \mathrm{H}), 3.46(\mathrm{~s}, 1 \mathrm{H}), 3.32(\mathrm{~s}, 3 \mathrm{H}), 3.26(\mathrm{~s}, 3 \mathrm{H}), 3.21(\mathrm{~s}, 3 \mathrm{H}), 3.15(\mathrm{~s}, 3 \mathrm{H}), 2.47-2.23$ (m, 4H), $2.21(\mathrm{dd}, J=12.0,4.3 \mathrm{~Hz}, 1 \mathrm{H}), 2.15(\mathrm{td}, J=12.4,3.6 \mathrm{~Hz}, 1 \mathrm{H}), 2.03(\mathrm{dd}, J=12.6,4.7 \mathrm{~Hz}, 1 \mathrm{H}), 1.99(\mathrm{dd}, J=$ 11.8, 4.1 Hz, 1H), 1.39 (d, J = 6.4 Hz, 3H), $1.25(\mathrm{~d}, J=6.5 \mathrm{~Hz}, 3 \mathrm{H}), 1.22(\mathrm{~d}, J=6.6 \mathrm{~Hz}, 3 \mathrm{H}), 1.03(\mathrm{~s}, 9 \mathrm{H}), 0.98(\mathrm{~d}, J=6.5$ $\mathrm{Hz}, 3 \mathrm{H}), 0.19(\mathrm{~s}, 6 \mathrm{H})$.

${ }^{13} \mathrm{C}$ NMR $\left(126 \mathrm{MHz}, \mathrm{C}_{6} \mathrm{D}_{6}\right) \delta 155.3,151.8,137.7,134.0,133.5,126.8,126.8,126.1,125.8,118.0,114.9,100.0$, 99.8, 98.6, 97.6, 77.6, 76.9, 76.6, 75.6, 75.3, 74.8, 74.3, 73.7, 68.4, 68.2, 67.9, 67.4, 56.1, 56.1, 55.8, 55.2, 34.7, $31.8,31.7,30.9,26.6,18.9,18.3,18.2,18.0,17.6,-3.9,-4.5$.

LRMS (ESI) m/z: (M+Na) ${ }^{+}$Calcd. $\mathrm{C}_{51} \mathrm{H}_{76} \mathrm{O}_{14} \mathrm{SiNa} 963.4902 ;$ Found 963.73

HRMS (ESI) m/z: (M+Na) ${ }^{+}$Calcd. $\mathrm{C}_{51} \mathrm{H}_{76} \mathrm{O}_{14} \mathrm{SiNa} 963.4902$; Found 963.4888

$[\alpha]_{\lambda}{ }^{24}\left(\mathrm{c}=4.7, \mathrm{CH}_{2} \mathrm{Cl}_{2}\right):-1.817$

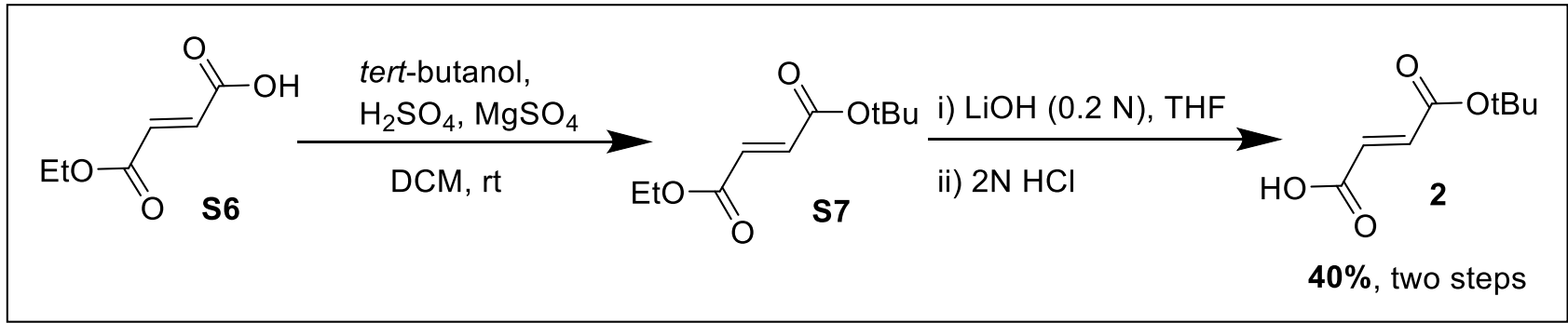

\section{tert-butyl fumarate (2)}

Following a literature protocol, ${ }^{4}$ concentrated sulfuric acid $(0.55 \mathrm{~mL}, 10 \mathrm{mmol})$ was added dropwise to a suspension of magnesium sulfate $(4.81 \mathrm{~g}, 40 \mathrm{mmol})$ in $\mathrm{CH}_{2} \mathrm{Cl}_{2}(40 \mathrm{~mL})$ and the suspension was stirred for 15 minutes. Monoethyl fumarate (S6, $1.44 \mathrm{~g}, 10 \mathrm{mmol}$ ) and tert-butanol $(4.78 \mathrm{~mL}, 50 \mathrm{mmol})$ were then added and the reaction was stirred at room temperature. After 18 hours, the reaction was quenched with $75 \mathrm{~mL}$ of saturated sodium bicarbonate solution. The aqueous phase was extracted with $\mathrm{CH}_{2} \mathrm{Cl}_{2}(3 \mathrm{x})$, and the pooled organic layers were then washed with brine $(1 \mathrm{x})$, dried over sodium sulfate, filtered through cotton, and concentrated under reduced pressure.

Following a literature protocol, ${ }^{5}$ the resulting crude tert-butyl monoethylfumarate $\mathbf{S 7}$ was then dissolved in $50 \mathrm{~mL}$ of THF and added slowly to a $0.2 \mathrm{~N}$ lithium hydroxide solution. After 30 minutes, the reaction was adjusted to $\mathrm{pH} \sim 2$ by dropwise addition of $2 \mathrm{~N} \mathrm{HCl}$ and diluted with $\mathrm{H}_{2} \mathrm{O}$. The aqueous phase was extracted with $\mathrm{Et}_{2} \mathrm{O}(3 \mathrm{x})$, and the pooled organic layers were then washed with brine $(1 \mathrm{x})$, dried over sodium sulfate, filtered through cotton, and concentrated under reduced pressure. The crude material was then purified by automated flash chromatography $(0 \%$ to $35 \%$ methanol: $\mathrm{CH}_{2} \mathrm{Cl}_{2}$ ) to afford tert-butyl fumarate 2 (698 mg, $40 \%$ over two steps) as a white amorphous solid. Spectroscopic data was in agreement with previously reported data. ${ }^{5}$

${ }^{1} \mathrm{H}$ NMR $\left(500 \mathrm{MHz}, \mathrm{CDCl}_{3}\right) \delta 6.87(\mathrm{~d}, J=15.7 \mathrm{~Hz}, 1 \mathrm{H}), 6.76(\mathrm{~d}, J=15.7 \mathrm{~Hz}, 1 \mathrm{H}), 1.52(\mathrm{~s}, 9 \mathrm{H})$.

${ }^{13} \mathrm{C}$ NMR $\left(126 \mathrm{MHz}, \mathrm{CDCl}_{3}\right) \delta 170.3,164.0,138.0,131.7,82.5,28.1$. 
${ }^{1} \mathrm{H}$ NMR (500 MHz, C $\left.6 \mathrm{D}_{6}\right) \delta 10.87(\mathrm{~s}, 1 \mathrm{H}, \mathrm{COOH}), 6.85(\mathrm{~d}, J=15.7 \mathrm{~Hz}, 1 \mathrm{H}), 6.78(\mathrm{~d}, J=15.7 \mathrm{~Hz}, 1 \mathrm{H}), 1.28(\mathrm{~s}, 9 \mathrm{H})$.

S2.9 Synthesis of AGM ester 1

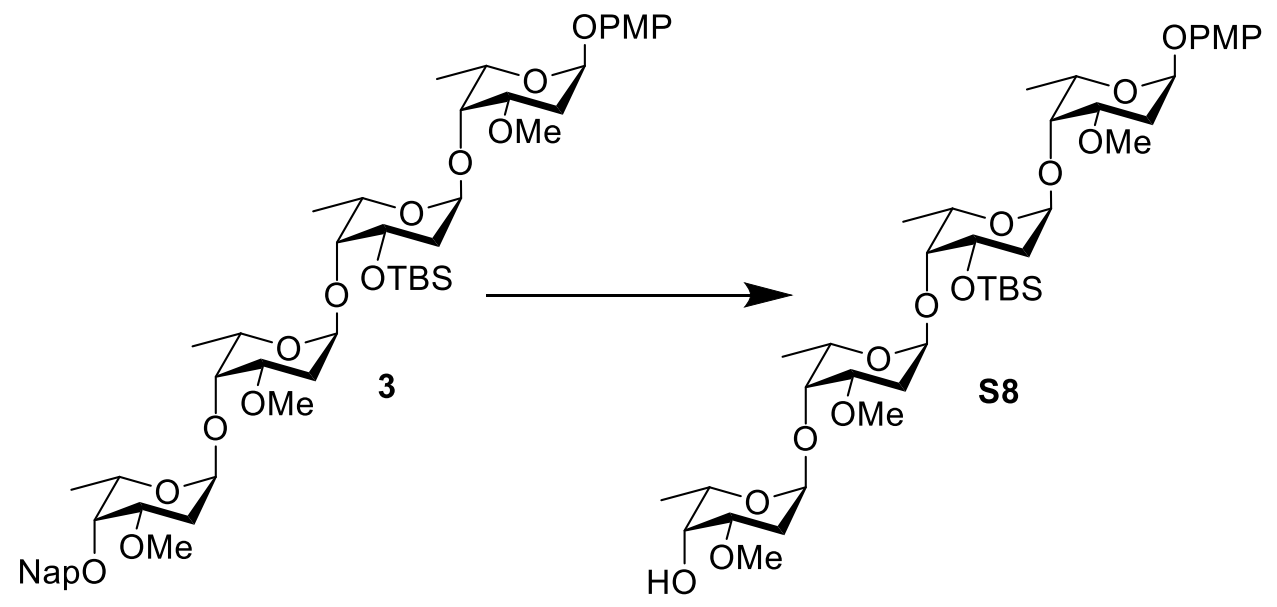

p-methoxyphenyl 2-deoxy-3-0-(methyl)-4-0-(2-deoxy-3-0-(tert-butyldimethylsilyl)-4-0-(2deoxy-3-0-methyl-4-0-(2-deoxy-3-0-(methyl)- $\alpha$-L-fucopyranosyl)- $\alpha$-L-fucopyranosyl)- $\alpha-L-$ fucopyranosyl)- $\alpha$-L-fucopyranoside (S8)

To a solution of compound 3 ( $42 \mathrm{mg}, 0.045 \mathrm{mmol})$ and $\beta$-Pinene $(24 \mu \mathrm{L}, 0.152 \mathrm{mmol})$ in $\mathrm{CH}_{2} \mathrm{Cl}_{2}(4.29 \mathrm{~mL})$ and $\mathrm{H}_{2} \mathrm{O}(0.22 \mathrm{~mL})$ was added solid DDQ $(226 \mathrm{mg}, 0.996 \mathrm{mmol})$. The reaction was stirred at room temperature for 2.5 hours, and then quenched with $10 \mathrm{~mL} 2 \mathrm{M}$ sodium hydroxide solution. The reaction was then diluted with $\mathrm{CH}_{2} \mathrm{Cl}_{2}$, and the organic layer was washed with $2 \mathrm{M}$ sodium hydroxide solution (3x). The pooled aqueous layers were back-extracted with $\mathrm{CH}_{2} \mathrm{Cl}_{2}(3 \mathrm{x})$, and the combined organic layers were then washed with brine (2x), dried over sodium sulfate, filtered through cotton, and concentrated under reduced pressure. The crude material was then purified by automated flash chromatography $(82 \%$ to $100 \%$ ethyl acetate:hexanes) and dried on high vacuum to afford tetrasaccharide alcohol $\mathbf{S 8}$ (38.6 mg, quantitative yield, $\alpha$-anomer only) as a clear oil.

${ }^{1} \mathrm{H}$ NMR $\left(500 \mathrm{MHz}, \mathrm{C}_{6} \mathrm{D}_{6}\right) \delta 7.11-7.04(\mathrm{~m}, 2 \mathrm{H}), 6.80-6.73(\mathrm{~m}, 2 \mathrm{H}), 5.42(\mathrm{~d}, J=3.4 \mathrm{~Hz}, 1 \mathrm{H}), 5.27(\mathrm{~d}, J=3.3 \mathrm{~Hz}, 1 \mathrm{H})$, $4.99(\mathrm{~d}, J=3.5 \mathrm{~Hz}, 1 \mathrm{H}), 4.94(\mathrm{~d}, J=3.3 \mathrm{~Hz}, 1 \mathrm{H}), 4.49(\mathrm{q}, J=6.5 \mathrm{~Hz}, 1 \mathrm{H}), 4.47-4.37(\mathrm{~m}, 3 \mathrm{H}), 3.84(\mathrm{~d}, J=10.2 \mathrm{~Hz}, 1 \mathrm{H})$, $3.79(\mathrm{~s}, 1 \mathrm{H}), 3.76-3.66(\mathrm{~m}, 4 \mathrm{H}), 3.63(\mathrm{~s}, 1 \mathrm{H}), 3.54(\mathrm{~s}, 1 \mathrm{H}), 3.32(\mathrm{~s}, 3 \mathrm{H}), 3.25(\mathrm{~s}, 3 \mathrm{H}), 3.21(\mathrm{~s}, 3 \mathrm{H}), 2.97(\mathrm{~s}, 3 \mathrm{H}), 2.38(\mathrm{td}$, $J=12.0,3.4 \mathrm{~Hz}, 1 \mathrm{H}), 2.27(\mathrm{td}, J=12.1,3.5 \mathrm{~Hz}, 1 \mathrm{H}), 2.22-1.95(\mathrm{~m}, 7 \mathrm{H}), 1.49(\mathrm{~d}, J=6.5 \mathrm{~Hz}, 3 \mathrm{H}), 1.24(\mathrm{~d}, J=6.5 \mathrm{~Hz}, 3 \mathrm{H})$, $1.21(\mathrm{~d}, J=6.6 \mathrm{~Hz}, 3 \mathrm{H}), 1.03(\mathrm{~s}, 9 \mathrm{H}), 0.98(\mathrm{~d}, J=6.5 \mathrm{~Hz}, 3 \mathrm{H}), 0.19(\mathrm{~s}, 6 \mathrm{H})$.

${ }^{13} \mathrm{C}$ NMR $\left(126 \mathrm{MHz}, \mathrm{C}_{6} \mathrm{D}_{6}\right) \delta 155.3,151.8,118.0,114.9,99.8,99.7,98.6,97.6,76.9,75.6,75.3,75.1,74.6,73.7$, $68.4,68.2,67.9,67.9,67.3,66.2,56.1,56.1,55.2,54.9,34.7,31.8,31.7,30.2,26.6,18.9,18.3,18.2,17.6,17.5$, $3.9,-4.5$.

LRMS (ESI) m/z: (M+Na) $)^{+}$Calcd. $\mathrm{C}_{40} \mathrm{H}_{68} \mathrm{O}_{14} \mathrm{SiNa} 823.4276$; Found 823.64

HRMS (ESI) m/z: (M+Na) ${ }^{+}$Calcd. $\mathrm{C}_{40} \mathrm{H}_{68} \mathrm{O}_{14} \mathrm{SiNa}$ 823.4276; Found 823.4274

$[\alpha]_{\lambda}{ }^{24}\left(\mathrm{c}=0.3, \mathrm{CH}_{2} \mathrm{Cl}_{2}\right):-0.700$ 


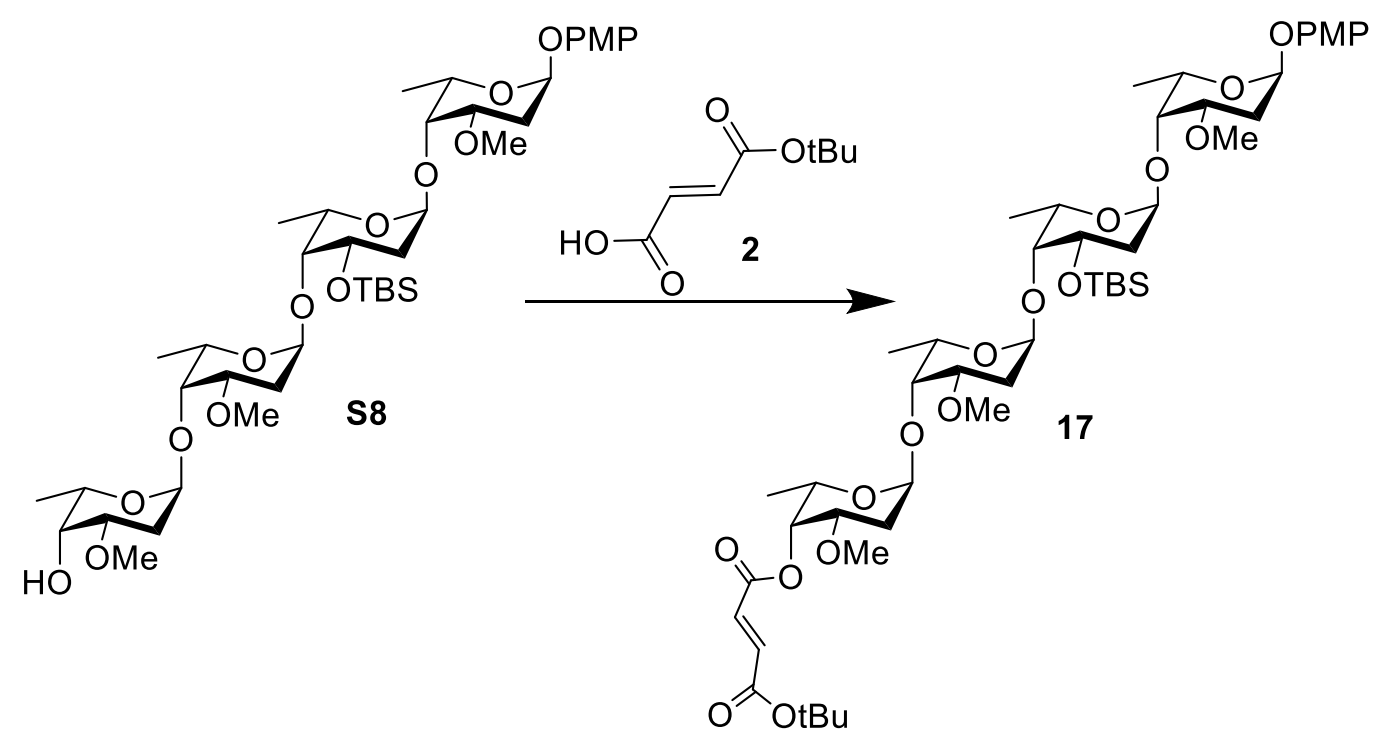

p-methoxyphenyl 2-deoxy-3-0-(methyl)-4-0-(2-deoxy-3-0-(tert-butyldimethylsilyl)-4-0-(2deoxy-3-0-methyl-4-0-(2-deoxy-3-0-(methyl)-4-0-(tert-butyl fumaryl)- $\alpha$-L-fucopyranosyl)- $\alpha-L-$ fucopyranosyl)- $\alpha$-L-fucopyranosyl)- $\alpha$-L-fucopyranoside (17)

In a flame-dried flask, deprotected tetrasaccharide $\mathbf{S 8}(35.73 \mathrm{mg}, 0.045 \mathrm{mmol})$, tert-butyl fumarate 2 (15.35 mg, $0.089 \mathrm{mmol}$ ), and DMAP (16.35 mg, $0.134 \mathrm{mmol}$ ) were dissolved in $\mathrm{CH}_{2} \mathrm{Cl}_{2}$ $(0.35 \mathrm{~mL}) . \mathrm{EDCl} \cdot \mathrm{HCl}(25.65 \mathrm{mg}, 0.134 \mathrm{mmol})$ and DIPEA $(23.3 \mu \mathrm{L}, 0.139 \mathrm{mmol})$ were then added and the reaction was stirred at room temperature. After 140 minutes, the reaction as quenched with $3 \mathrm{~mL}$ saturated sodium bicarbonate solution and diluted with $\mathrm{H}_{2} \mathrm{O}$ and $\mathrm{CH}_{2} \mathrm{Cl}_{2}$. The aqueous phase was extracted with $\mathrm{CH}_{2} \mathrm{Cl}_{2}(5 \mathrm{x})$, and the pooled organic layers were then washed with brine $(1 \mathrm{x})$, dried over sodium sulfate, filtered through cotton, and concentrated under reduced pressure. The crude material was purified by automated flash chromatography ( $22 \%$ to $63 \%$ ethyl acetate:hexanes) to afford tetrasaccharide ester 17 (31.6 $\mathrm{mg}, 74 \%)$ as a yellow oil.

${ }^{1} \mathrm{H}$ NMR $\left(500 \mathrm{MHz}, \mathrm{C}_{6} \mathrm{D}_{6}\right) \delta 7.15-7.04(\mathrm{~m}, 4 \mathrm{H}), 6.80-6.73(\mathrm{~m}, 2 \mathrm{H}), 5.42(\mathrm{~d}, J=3.3 \mathrm{~Hz}, 1 \mathrm{H}), 5.39(\mathrm{~s}, 1 \mathrm{H}), 5.28(\mathrm{~s}, 1 \mathrm{H})$, $4.98-4.90(\mathrm{~m}, 2 \mathrm{H}), 4.57(\mathrm{q}, J=7.0,6.5 \mathrm{~Hz}, 1 \mathrm{H}), 4.49-4.37(\mathrm{~m}, 3 \mathrm{H}), 3.86-3.66(\mathrm{~m}, 6 \mathrm{H}), 3.55(\mathrm{~d}, J=2.7 \mathrm{~Hz}, 1 \mathrm{H})$, $3.32(\mathrm{~s}, 3 \mathrm{H}), 3.22(\mathrm{~s}, 3 \mathrm{H}), 3.18(\mathrm{~s}, 3 \mathrm{H}), 3.15(\mathrm{~s}, 3 \mathrm{H}), 2.38(\mathrm{td}, J=12.0,3.4 \mathrm{~Hz}, 1 \mathrm{H}), 2.27-2.10(\mathrm{~m}, 4 \mathrm{H}), 2.09-1.96(\mathrm{~m}$, $3 \mathrm{H}), 1.27(\mathrm{~s}, 9 \mathrm{H}), 1.25-1.18(\mathrm{~m}, 9 \mathrm{H}), 1.03(\mathrm{~s}, 9 \mathrm{H}), 0.98(\mathrm{~d}, J=6.5 \mathrm{~Hz}, 3 \mathrm{H}), 0.20(\mathrm{~s}, 6 \mathrm{H})$.

${ }^{13} \mathrm{C}$ NMR $\left(126 \mathrm{MHz}, \mathrm{C}_{6} \mathrm{D}_{6}\right) \delta 165.1,164.0,155.3,151.8,136.0,133.1,118.0,114.9,99.8,99.7,98.6,97.6,81.1$, 77.0, 75.5, 75.4, 75.0, 73.9, 73.8, 70.3, 68.4, 68.2, 67.9, 67.2, 65.3, 56.1, 56.1, 55.9, 55.2, 34.7, 31.8, 31.6, 27.8, $26.6,18.9,18.3,18.1,17.6,17.2,-3.9,-4.5$.

LRMS (ESI) m/z: (M+Na) ${ }^{+}$Calcd. $\mathrm{C}_{48} \mathrm{H}_{78} \mathrm{O}_{17} \mathrm{SiNa} 977.4906$; Found 977.73

HRMS (ESI) m/z: (M+Na)+ Calcd. $\mathrm{C}_{48} \mathrm{H}_{78} \mathrm{O}_{17} \mathrm{SiNa} 977.4906$; Found 977.4896

$[\alpha]_{\lambda}{ }^{24}\left(\mathrm{c}=1.2, \mathrm{CH}_{2} \mathrm{Cl}_{2}\right):-0.072$ 


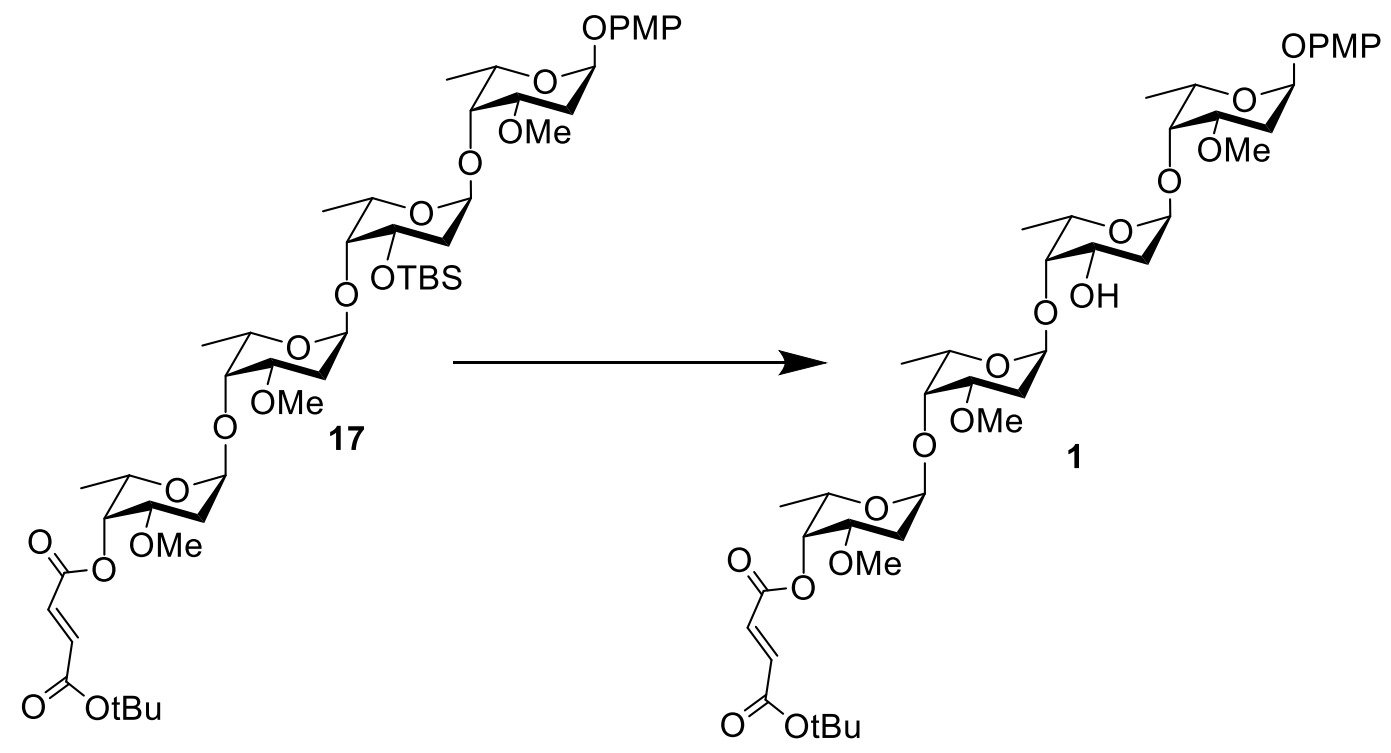

p-methoxyphenyl 2-deoxy-3-0-(methyl)-4-0-(2-deoxy-4-0-(2-deoxy-3-0-methyl-4-0-(2deoxy-3-O-(methyl)-4-O-(tert-butyl fumaryl)- $\alpha$-L-fucopyranosyl)- $\alpha$-L-fucopyranosyl)- $\alpha-L-$ fucopyranosyl)- $\alpha$-L-fucopyranoside (1)

Into a flame-dried flask containing ester 17 (16 mg, $0.017 \mathrm{mmol}$ ) was added $0.27 \mathrm{~mL}$ of a $0.2 \mathrm{M}$ solution of $3 \mathrm{HF} \cdot \mathrm{Et}_{3} \mathrm{~N}$ in acetonitrile and the reaction was monitored by $\mathrm{TLC}$ at room temperature for consumption of 17. A total of 299 equivalents of neat $3 \mathrm{HF} \cdot \mathrm{Et}_{3} \mathrm{~N}(0.82 \mathrm{~mL}, 5.03$ $\mathrm{mmol})$ were added over a period of 46 hours to drive the reaction to completion. The reaction was quenched at hour 46 with $4 \mathrm{~mL}$ saturated sodium bicarbonate solution and allowed to stir for 20 minutes. The reaction was diluted with $\mathrm{H}_{2} \mathrm{O}$ and ethyl acetate, and the organic phase was washed with saturated sodium bicarbonate solution. The aqueous phase was then extracted with ethyl acetate $(3 x)$, and the pooled organic layers were then washed with brine $(1 x)$, dried over sodium sulfate, filtered through cotton, and concentrated under reduced pressure. The crude material was purified by automated flash chromatography ( $40 \%$ to $82 \%$ ethyl acetate:hexanes) to afford tetrasaccharide alcohol ester $1(9 \mathrm{mg}, 64 \%)$ as a beige oil.

${ }^{1} \mathrm{H}$ NMR $\left(500 \mathrm{MHz}, \mathrm{C}_{6} \mathrm{D}_{6}\right) \delta 7.15-7.14(\mathrm{~m}, 2 \mathrm{H}), 7.11(\mathrm{~d}, J=5.1 \mathrm{~Hz}, 2 \mathrm{H}), 6.83-6.76(\mathrm{~m}, 2 \mathrm{H}), 5.48(\mathrm{~d}, J=3.4 \mathrm{~Hz}, 1 \mathrm{H})$, $5.37(\mathrm{~s}, 1 \mathrm{H}), 4.99(\mathrm{~d}, J=3.4 \mathrm{~Hz}, 1 \mathrm{H}), 4.82(\mathrm{~d}, J=3.7 \mathrm{~Hz}, 1 \mathrm{H}), 4.80(\mathrm{~d}, J=3.5 \mathrm{~Hz}, 1 \mathrm{H}), 4.53-4.43(\mathrm{~m}, 2 \mathrm{H}), 4.36-4.27$ $(\mathrm{m}, 1 \mathrm{H}), 4.00-3.89(\mathrm{~m}, 2 \mathrm{H}), 3.80-3.69(\mathrm{~m}, 3 \mathrm{H}), 3.62-3.55(\mathrm{~m}, 2 \mathrm{H}), 3.54(\mathrm{~s}, 1 \mathrm{H}), 3.33(\mathrm{~s}, 3 \mathrm{H}), 3.24(\mathrm{~s}, 3 \mathrm{H}), 3.21(\mathrm{~s}$, $1 \mathrm{H}), 3.17(\mathrm{~s}, 3 \mathrm{H}), 3.09(\mathrm{~s}, 3 \mathrm{H}), 2.33(\mathrm{dd}, J=12.1,4.6 \mathrm{~Hz}, 1 \mathrm{H}), 2.19(\mathrm{td}, J=12.5,3.7 \mathrm{~Hz}, 2 \mathrm{H}), 2.07$ (dd, $J=12.5,5.0 \mathrm{~Hz}$, $1 \mathrm{H}), 2.04-1.92(\mathrm{~m}, 4 \mathrm{H}), 1.27(\mathrm{~s}, 9 \mathrm{H}), 1.22(\mathrm{~d}, J=6.4 \mathrm{~Hz}, 3 \mathrm{H}), 1.18(\mathrm{~d}, J=6.5 \mathrm{~Hz}, 3 \mathrm{H}), 1.09-1.00(\mathrm{~m}, 6 \mathrm{H})$.

${ }^{13} \mathrm{C}$ NMR $\left(126 \mathrm{MHz}, \mathrm{C}_{6} \mathrm{D}_{6}\right) \delta 165.0,164.0,155.3,151.9,136.1,133.0,117.9,115.0,101.4,100.1,99.7,97.7,84.2$, $81.2,75.5,75.0,74.3,74.2,73.8,70.1,68.5,68.0,67.0,65.8,65.4,56.2,56.1,56.0,55.2,35.1,31.9,31.7,31.6$, $27.8,17.6,17.5,17.2$.

LRMS (ESI) m/z: (M+Na) ${ }^{+}$Calcd. $\mathrm{C}_{42} \mathrm{H}_{64} \mathrm{O}_{17} \mathrm{Na} 863.4041$; Found 863.55

HRMS (ESI) m/z: (M+Na) ${ }^{+}$Calcd. $\mathrm{C}_{42} \mathrm{H}_{64} \mathrm{O}_{17} \mathrm{Na} 863.4041$; Found 863.4023

$[\alpha]^{24}\left(\mathrm{c}=0.33, \mathrm{CH}_{2} \mathrm{Cl}_{2}\right):-0.309$ 


\section{References}

(1) Skornyakov, Y. V; Lozinskaya, N. A.; Proskurnina, M. V; Zefirov, N. S. Electrophilic Substitution in Aromatic and Heteroaromatic Substrates by Trichlorocyclopropenylium Tetrachloroaluminate. Russ. J. Org. Chem. 2005, 41, 689-693.

(2) Nogueira, J. M.; Bylsma, M.; Bright, D. K.; Bennett, C. S. Reagent-Controlled $\alpha$-Selective Dehydrative Glycosylation of 2,6-Dideoxy- and 2,3,6-Trideoxy Sugars. Angew. Chemie - Int. Ed. 2016, 55, 10088-10092.

(3) Zeng, J.; Sun, G.; Yao, W.; Zhu, Y.; Wang, R.; Cai, L.; Liu, K.; Zhang, Q.; Liu, X. W.; Wan, Q. 3Aminodeoxypyranoses in Glycosylation: Diversity-Oriented Synthesis and Assembly in Oligosaccharides. Angew. Chemie - Int. Ed. 2017, 56, 5227-5231.

(4) Wright, S. W.; Hageman, D. L.; Wright, A. S.; McClure, L. D. Convenient Preparations of TButyl Esters and Ethers from t-Butanol. Tetrahedron Lett. 1997, 38, 7345-7348.

(5) Denmark, S. E.; Thorarensen, A.; Middleton, D. S. Tandem [4+2]/[3+2] Cycloadditions of Nitroalkenes. 9. Synthesis of (-)-Rosmarinecine. J. Am. Chem. Soc. 1996, 118, 8266-8277.

\section{NMR Spectra}




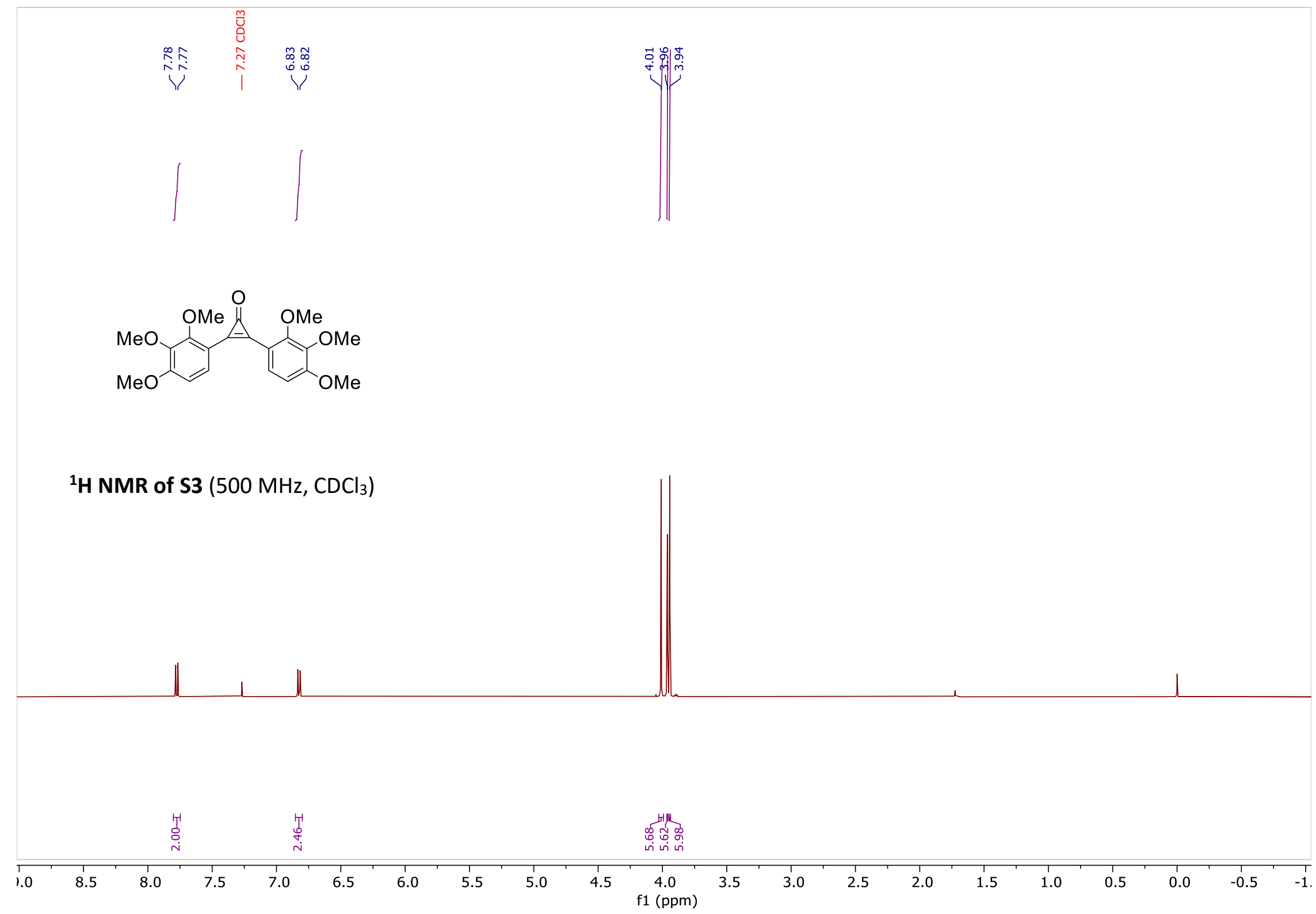



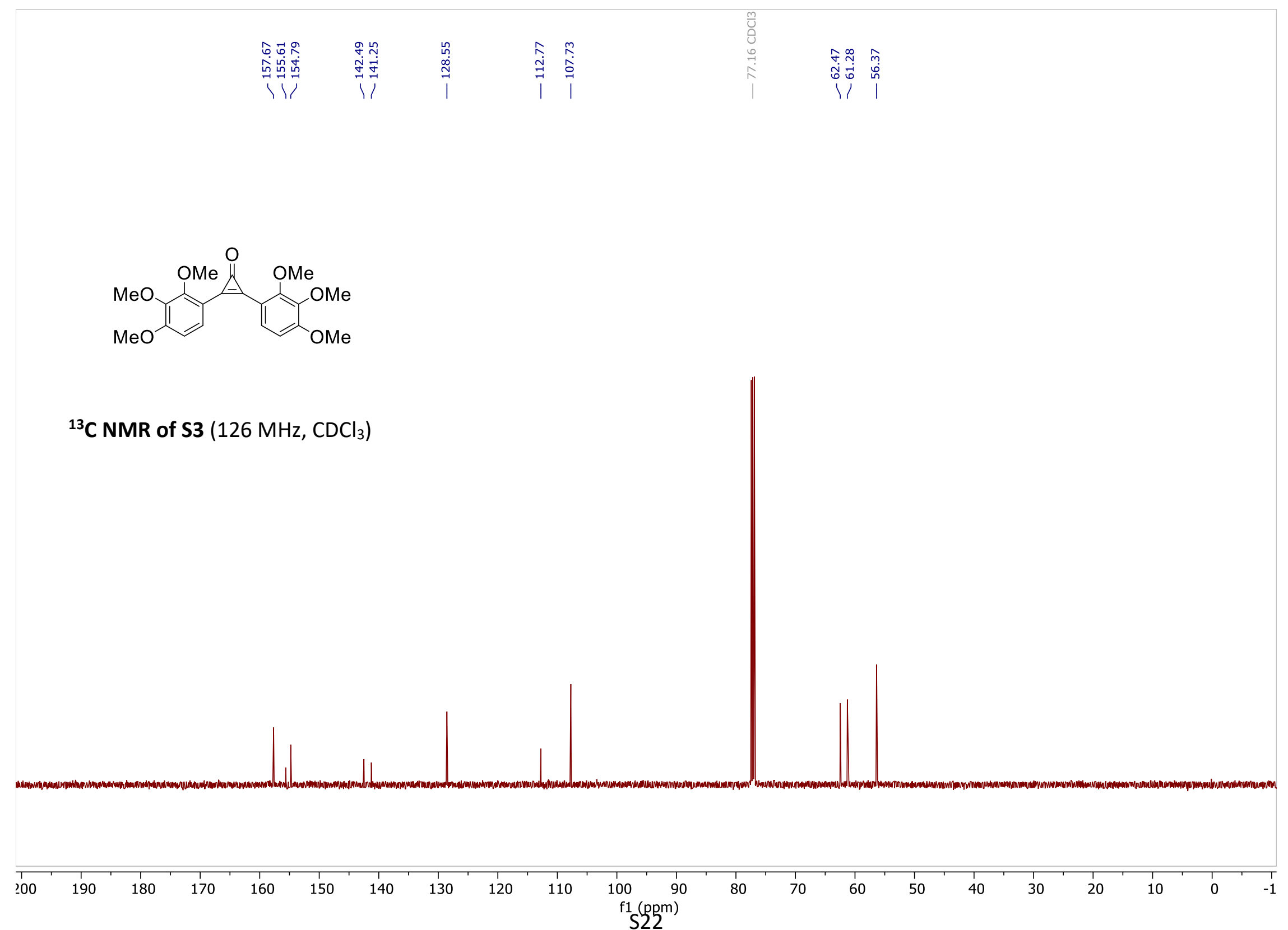


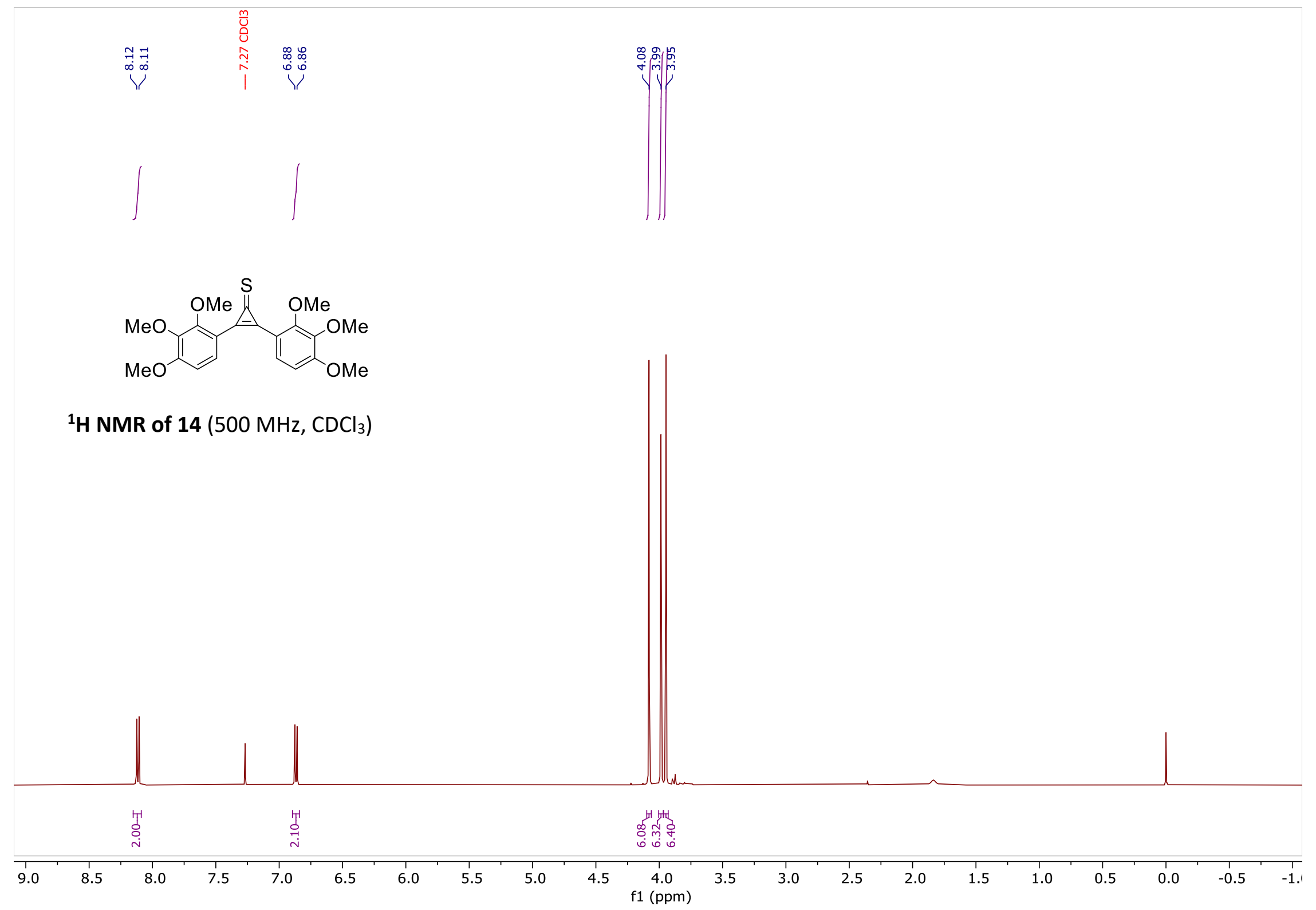




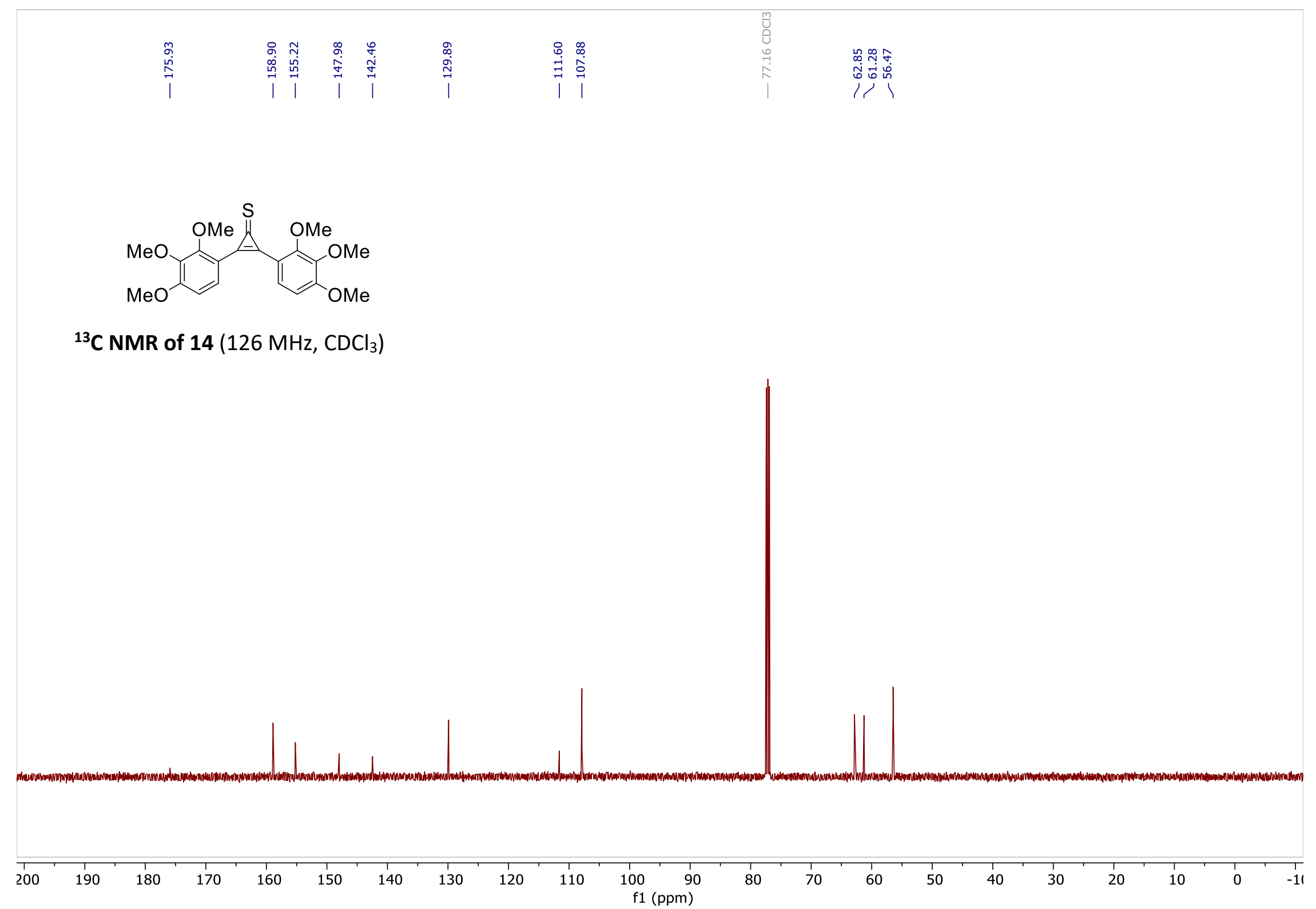




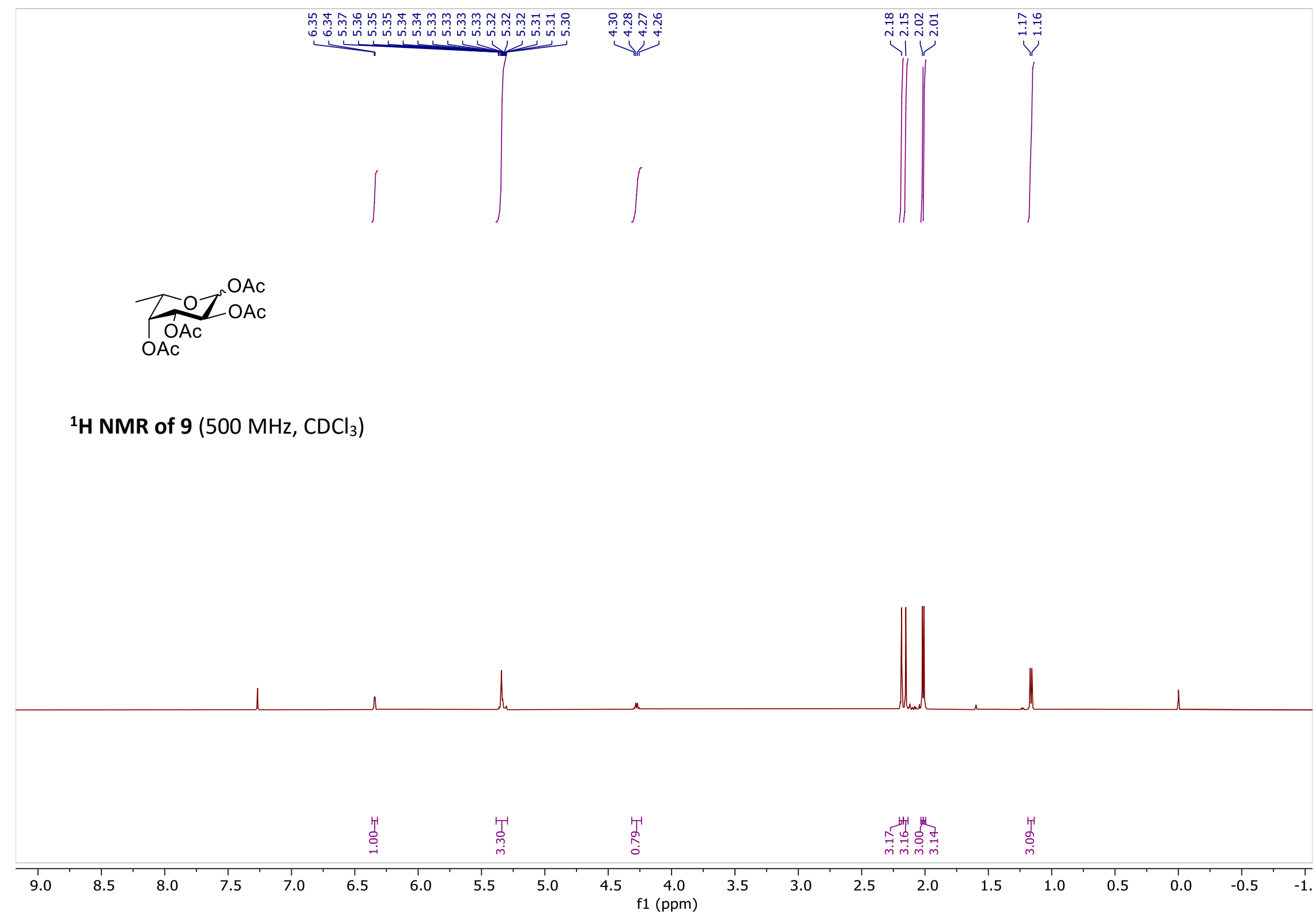




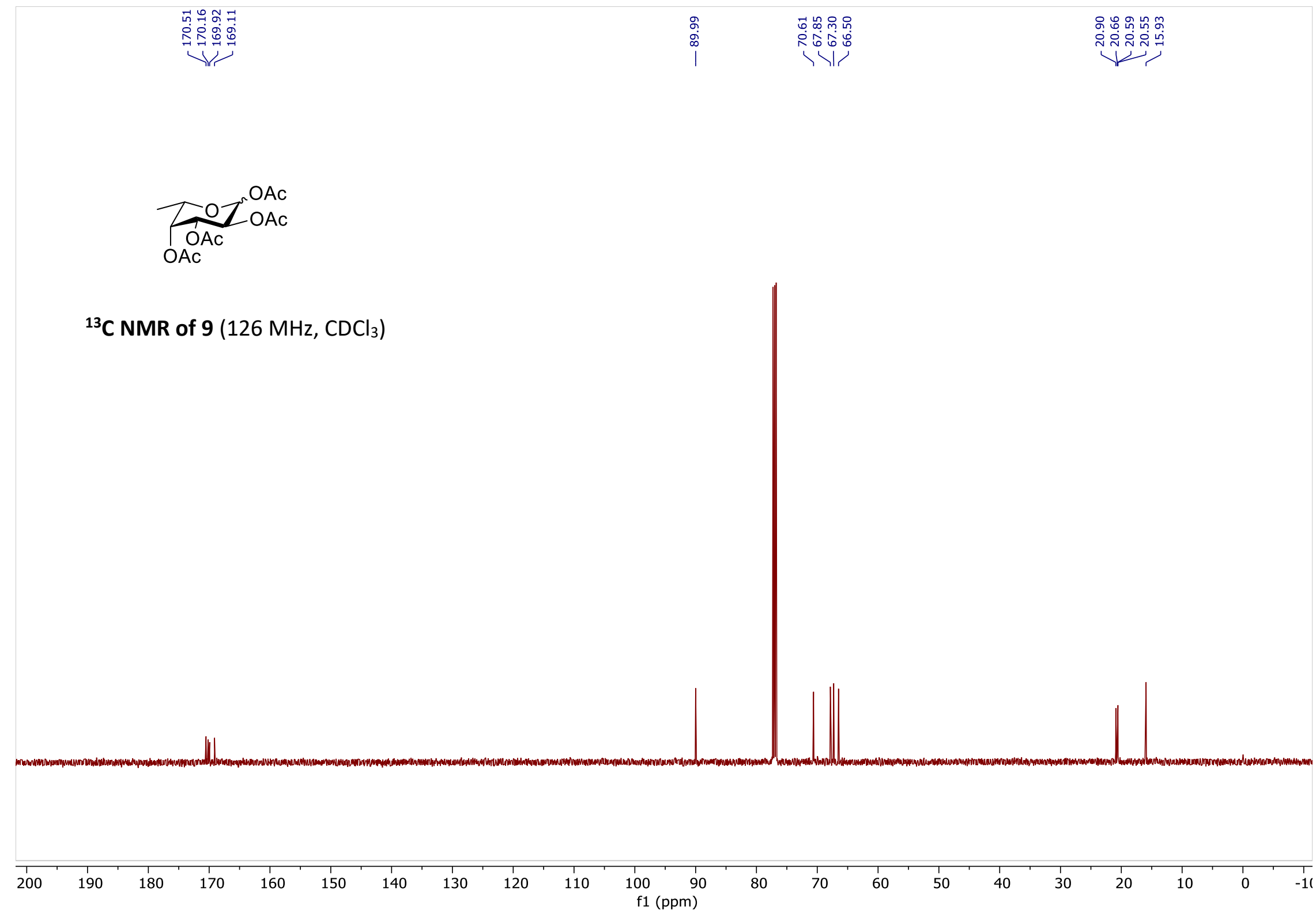




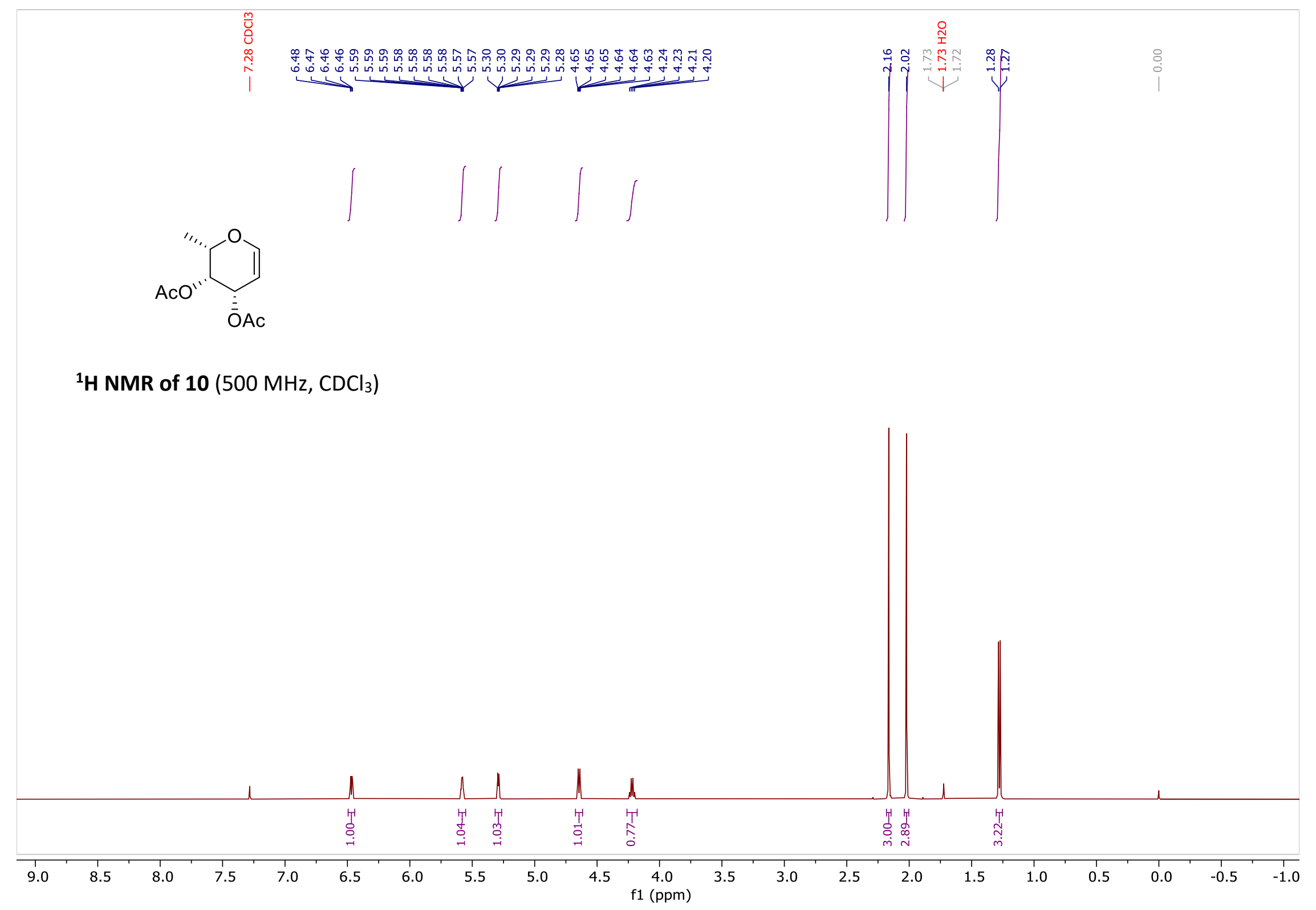




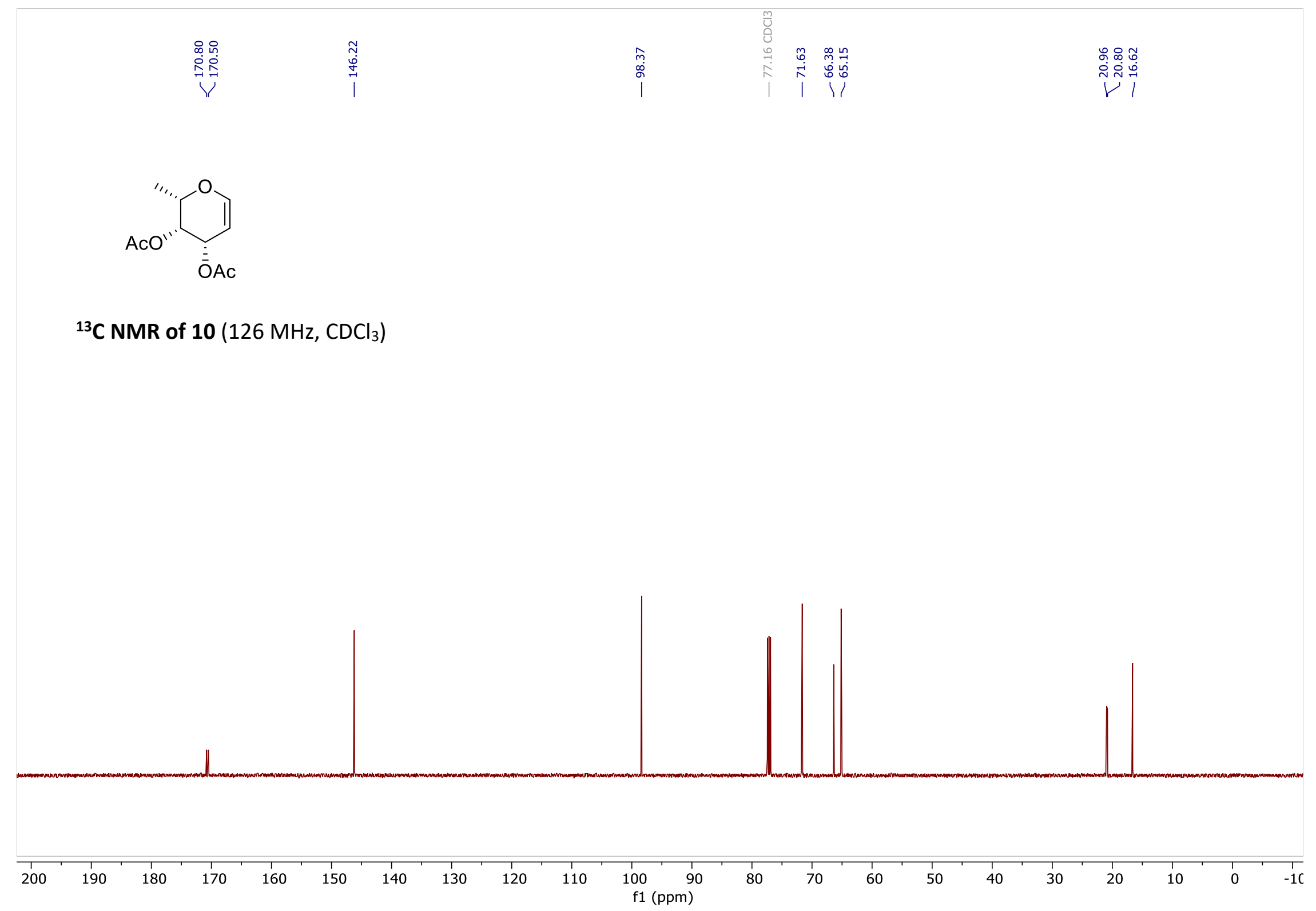




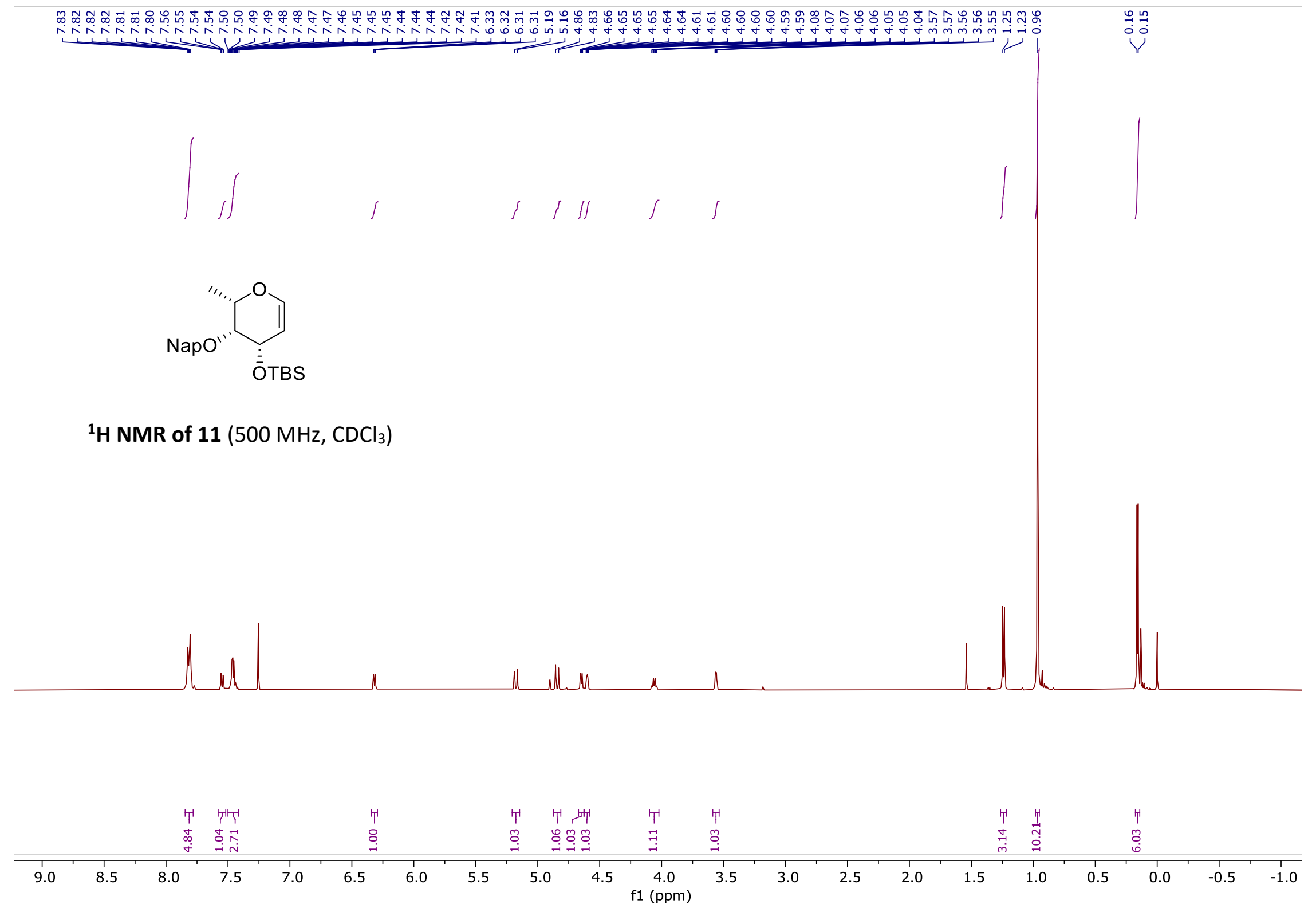




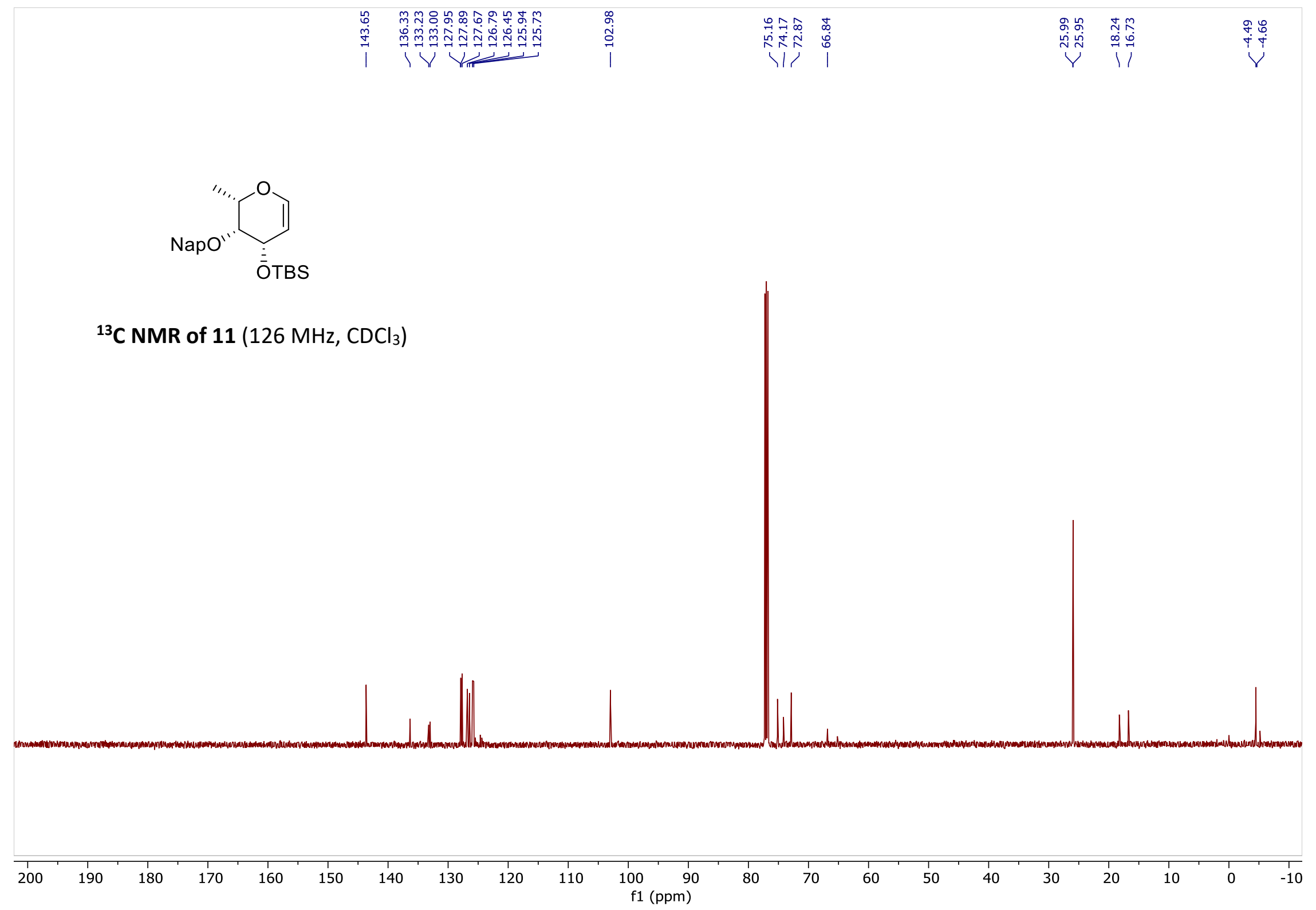



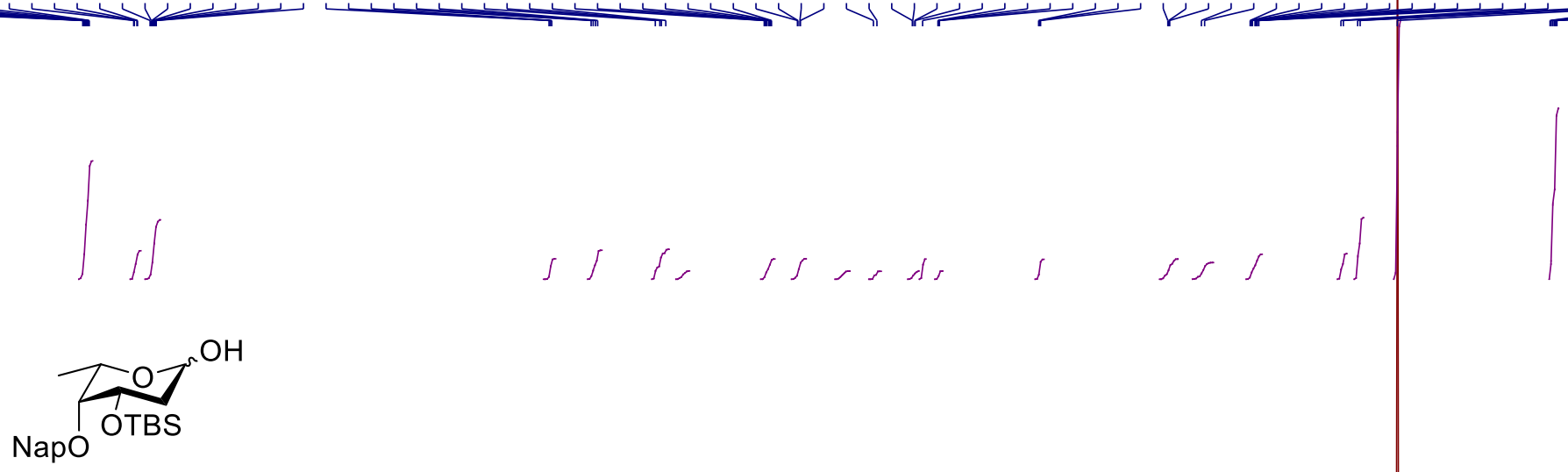

${ }^{1} \mathbf{H}$ NMR of $7\left(500 \mathrm{MHz}, \mathrm{CDCl}_{3}\right)$

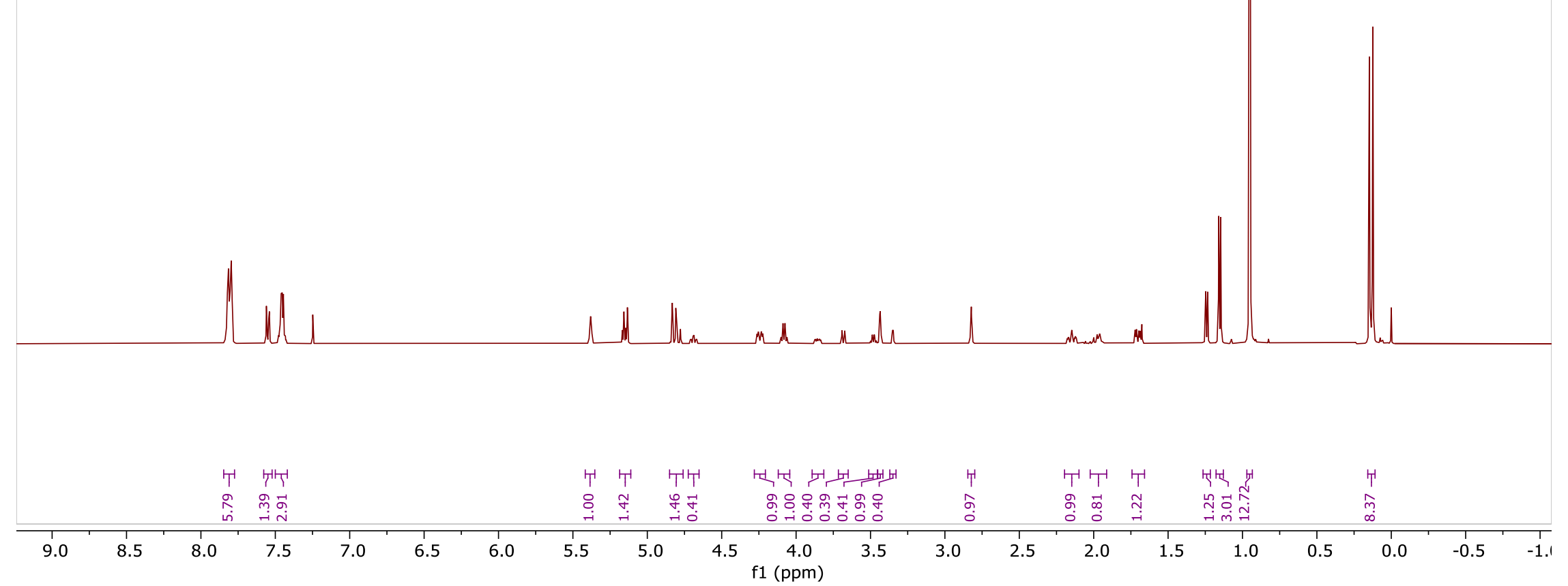




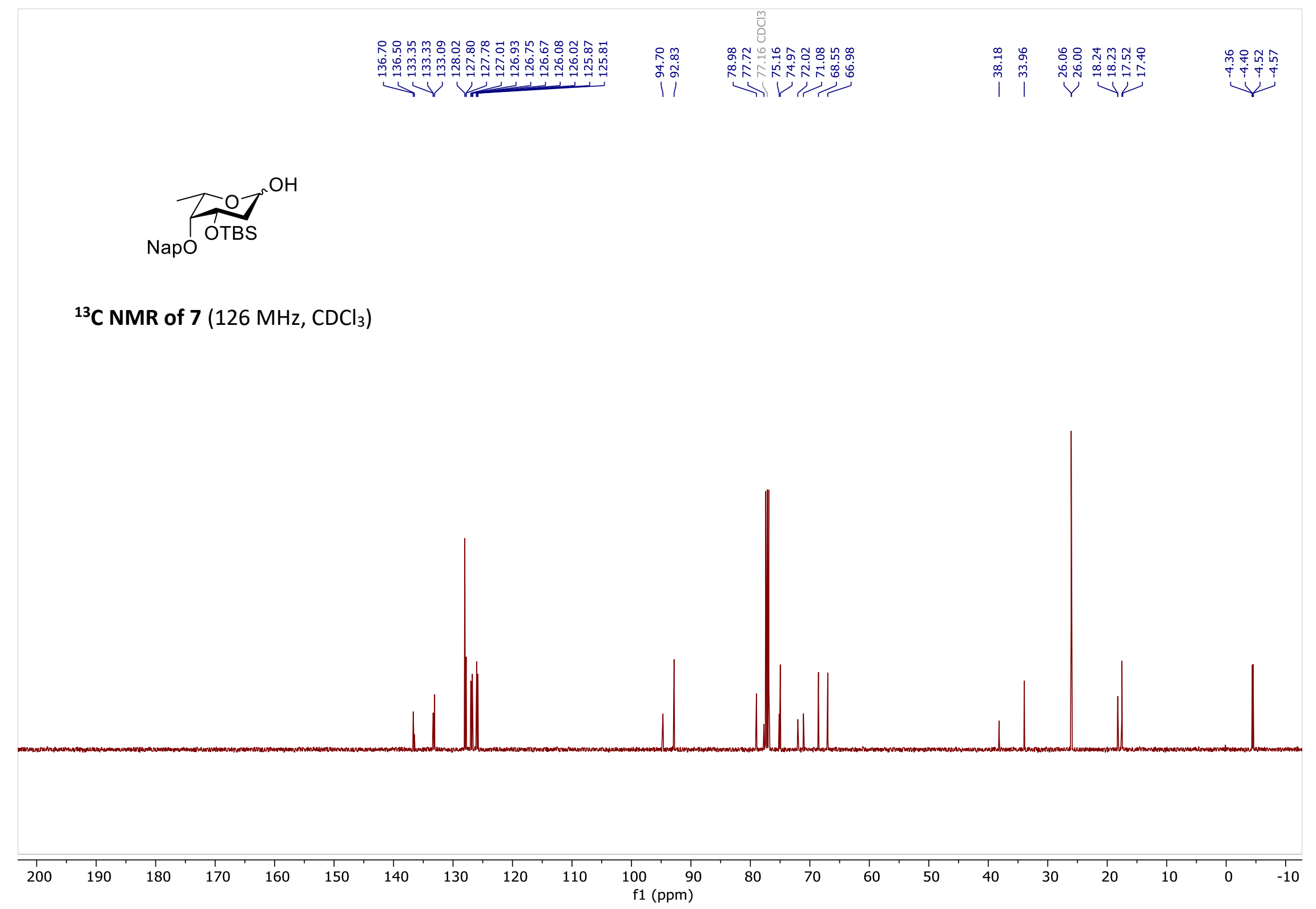




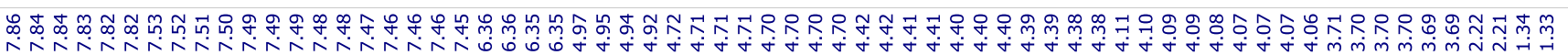
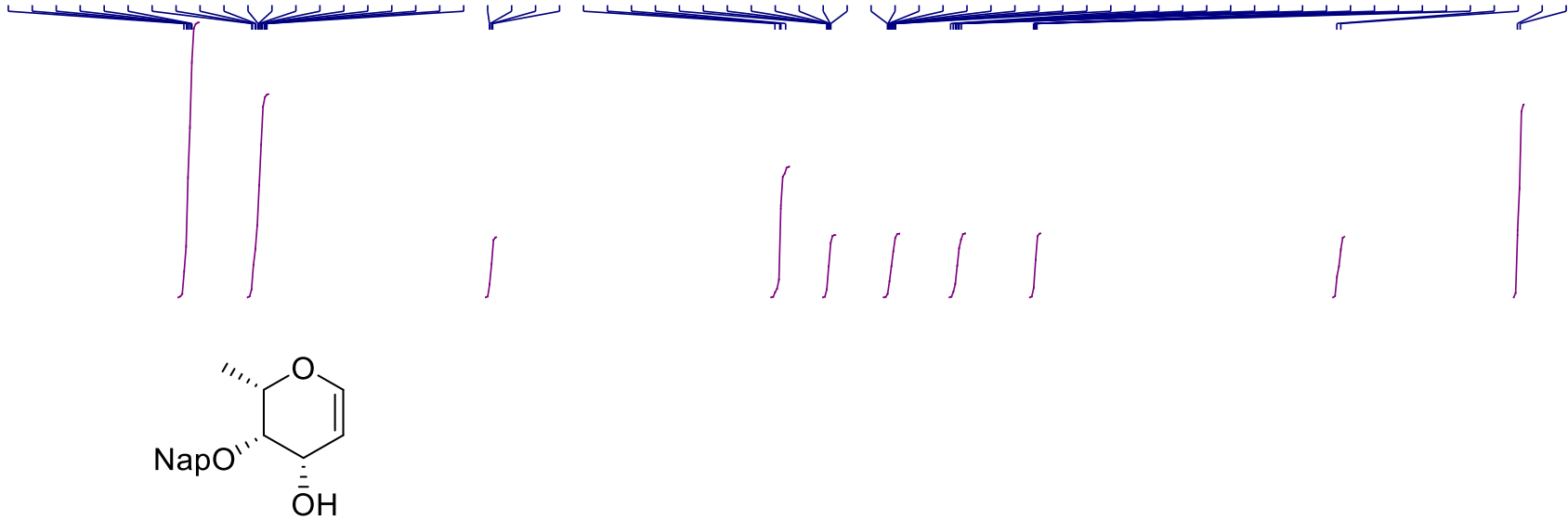

${ }^{1} \mathrm{H}$ NMR of $12\left(500 \mathrm{MHz}, \mathrm{CDCl}_{3}\right)$

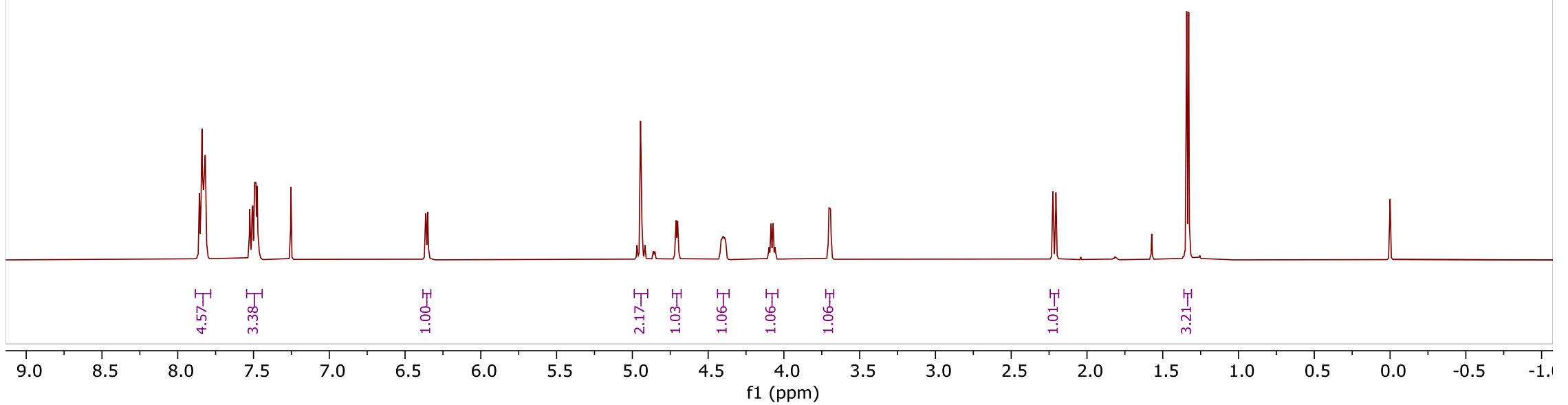




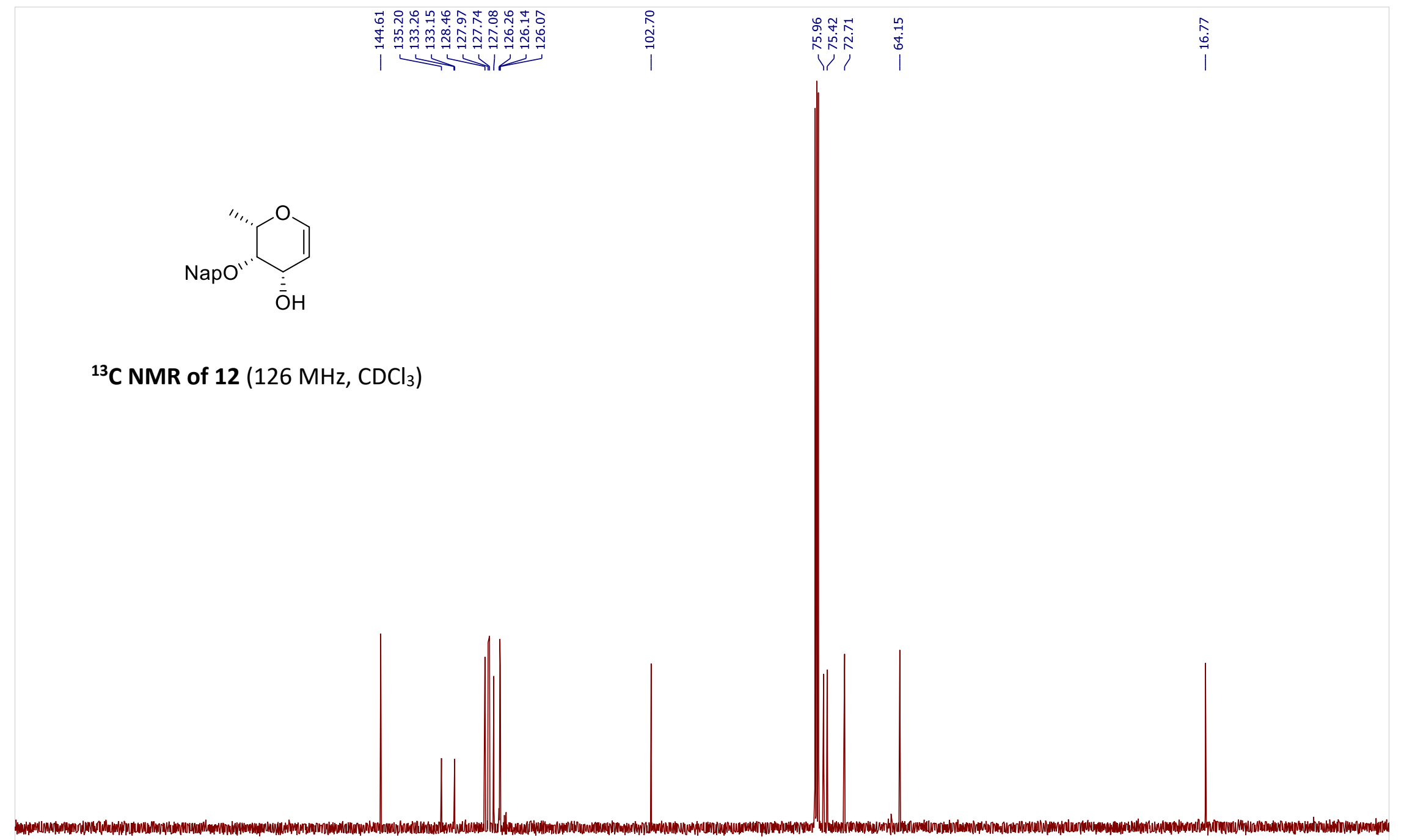

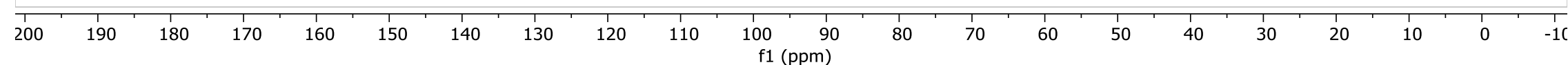




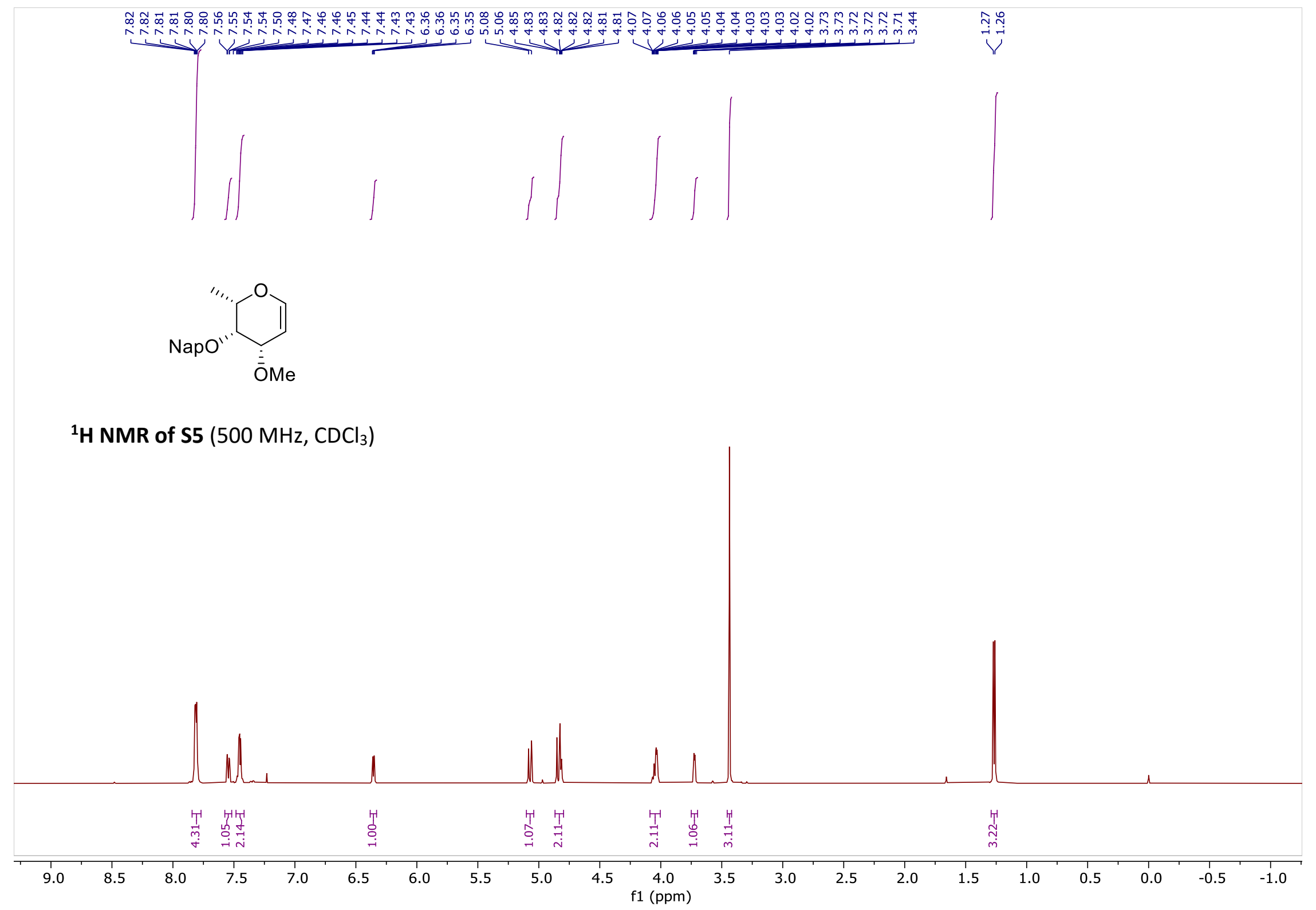




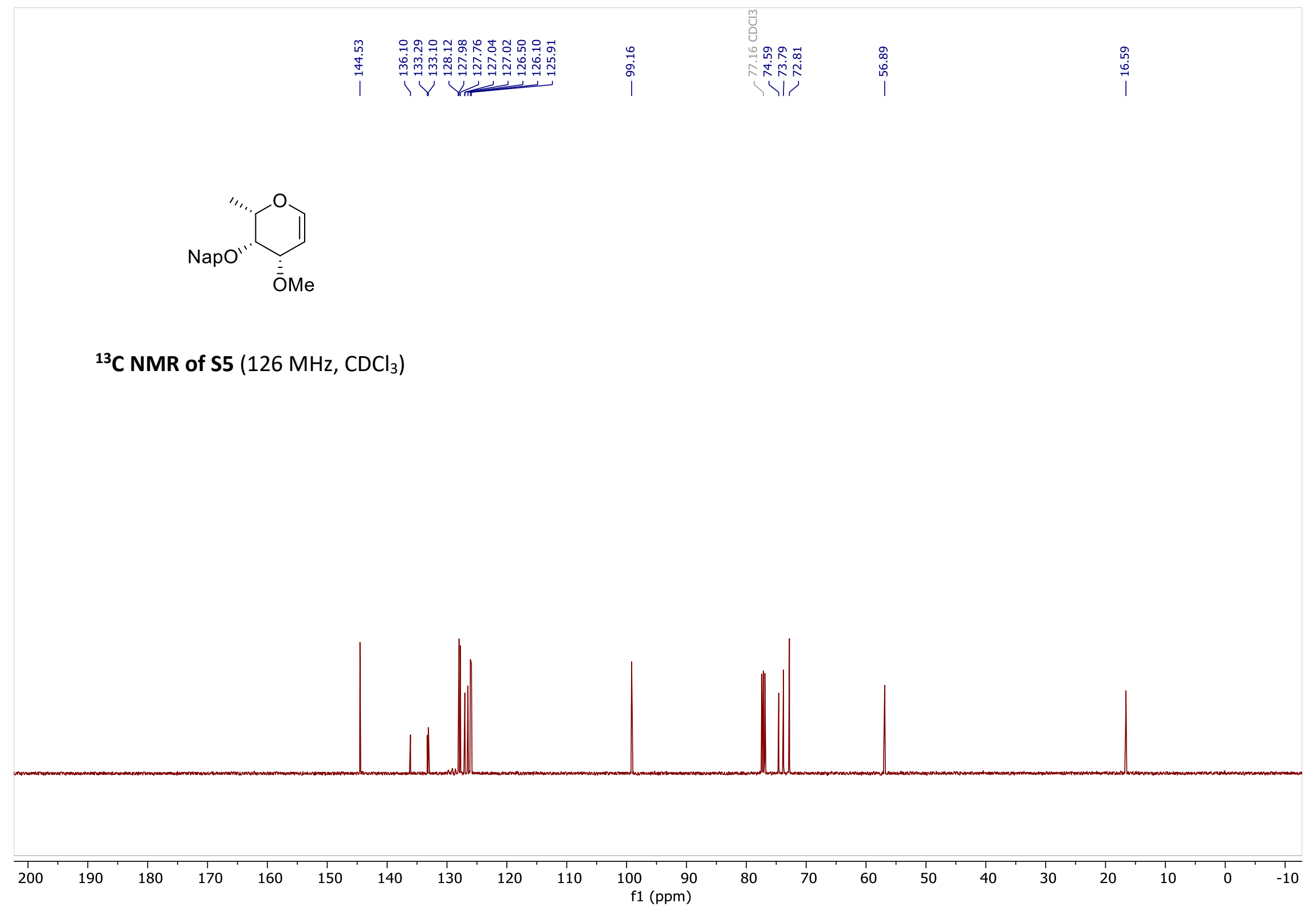




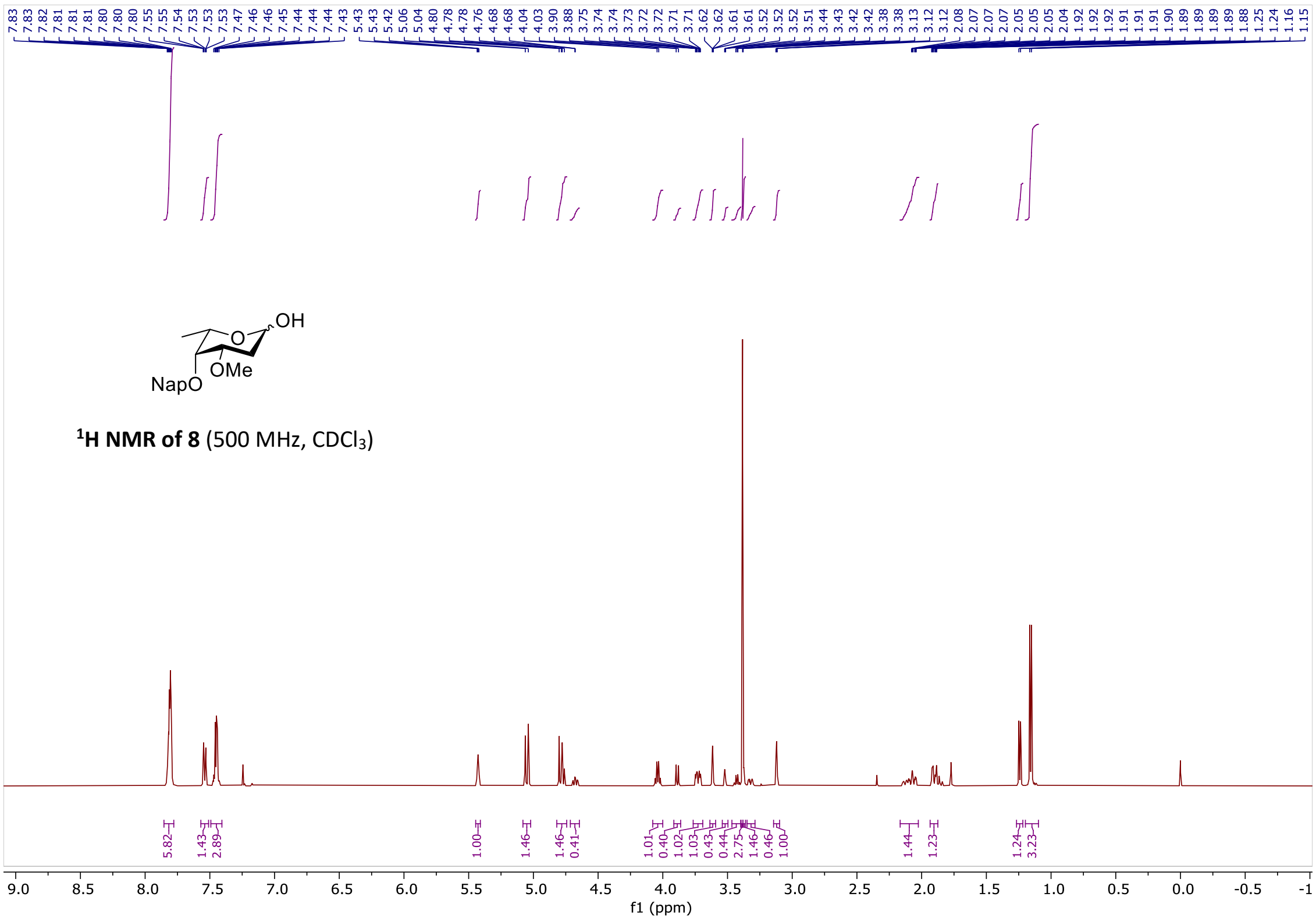




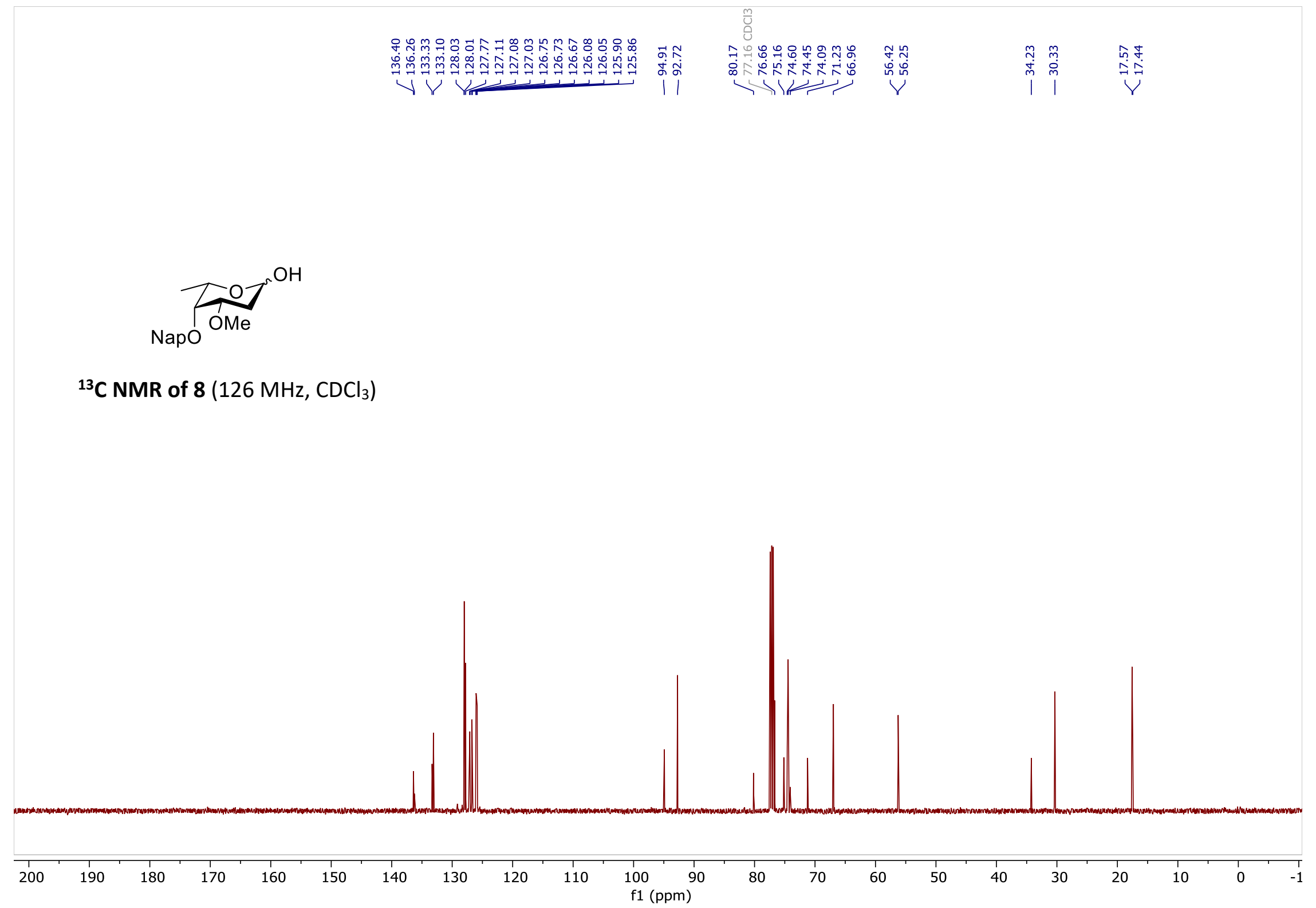




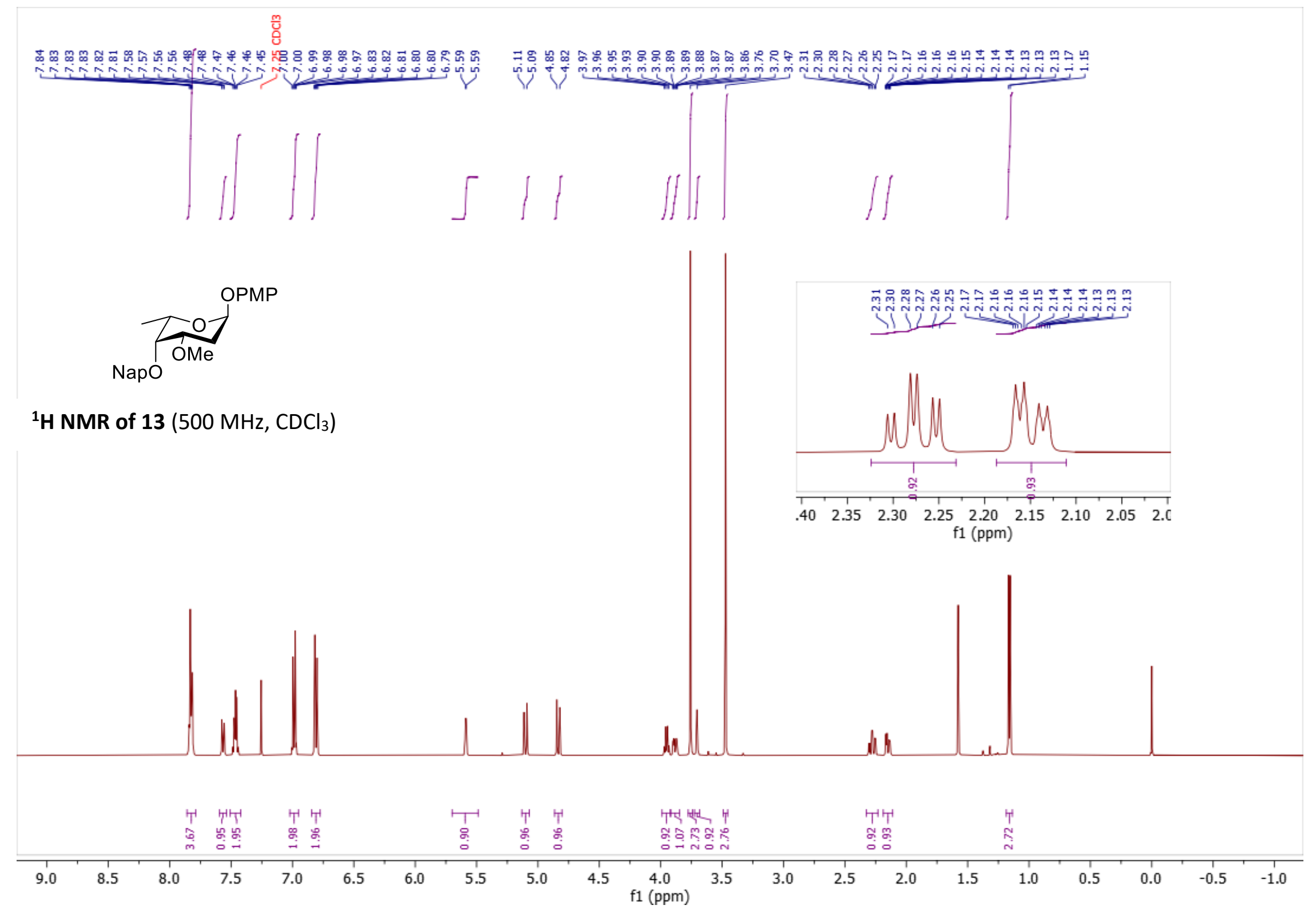




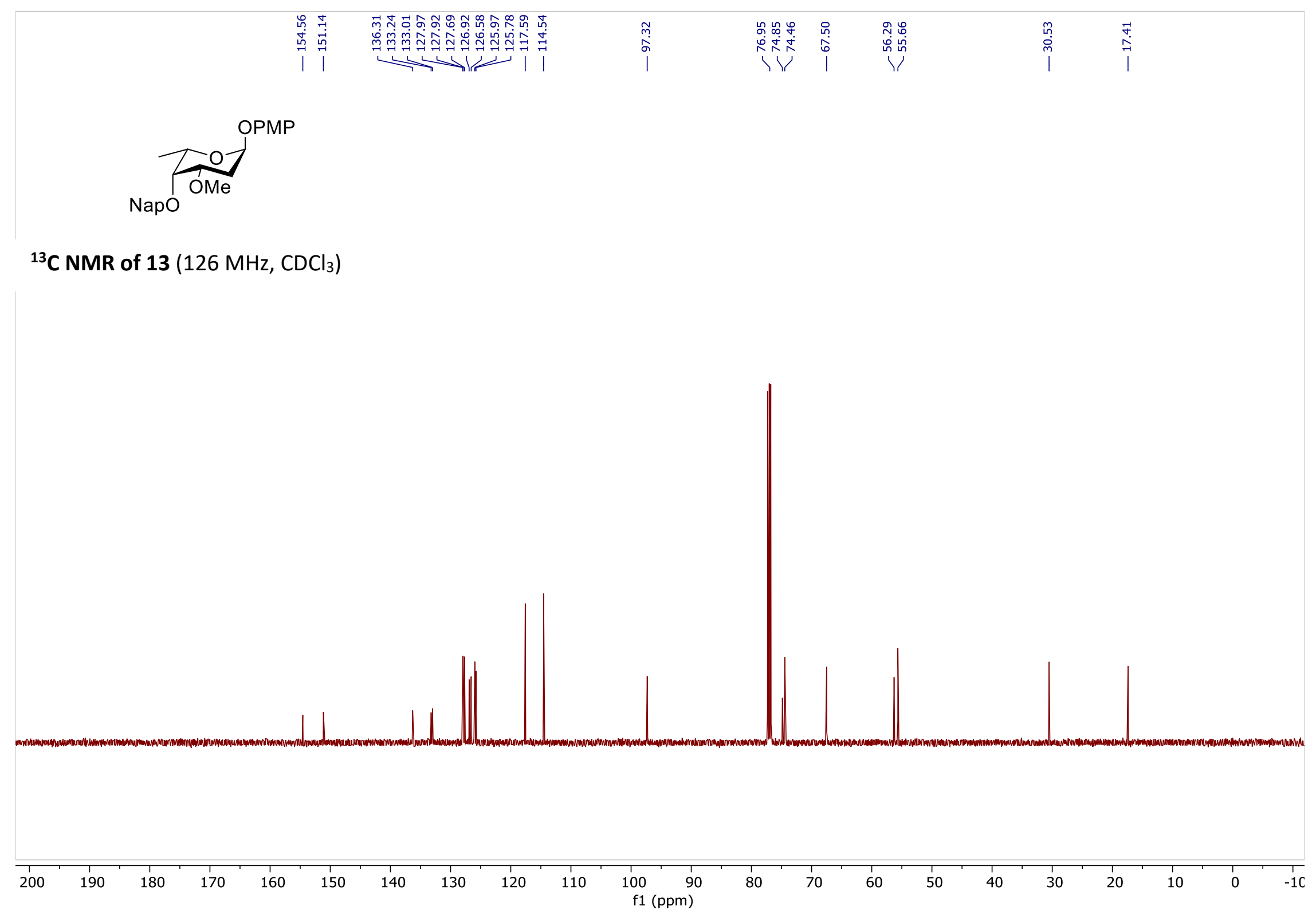




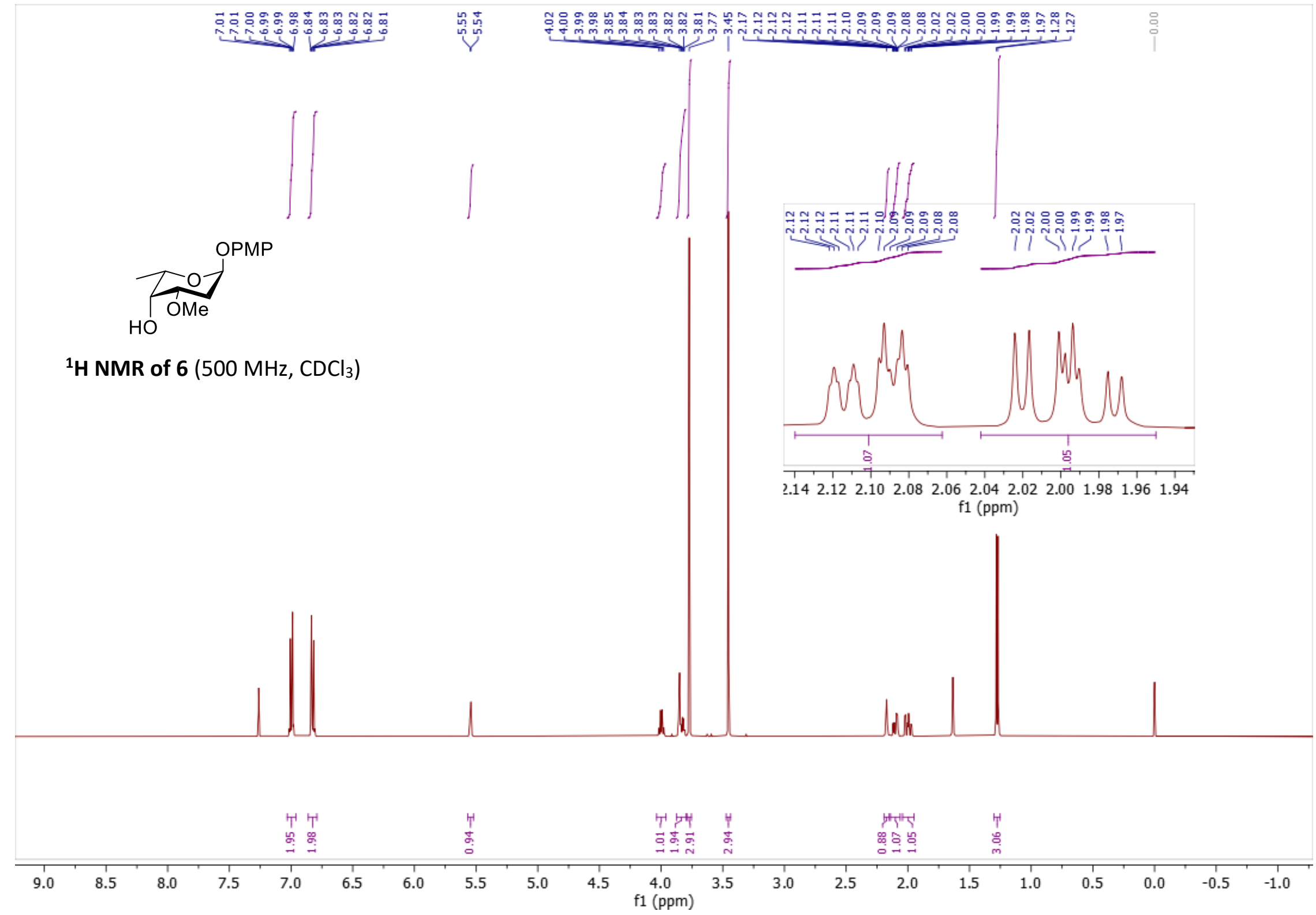




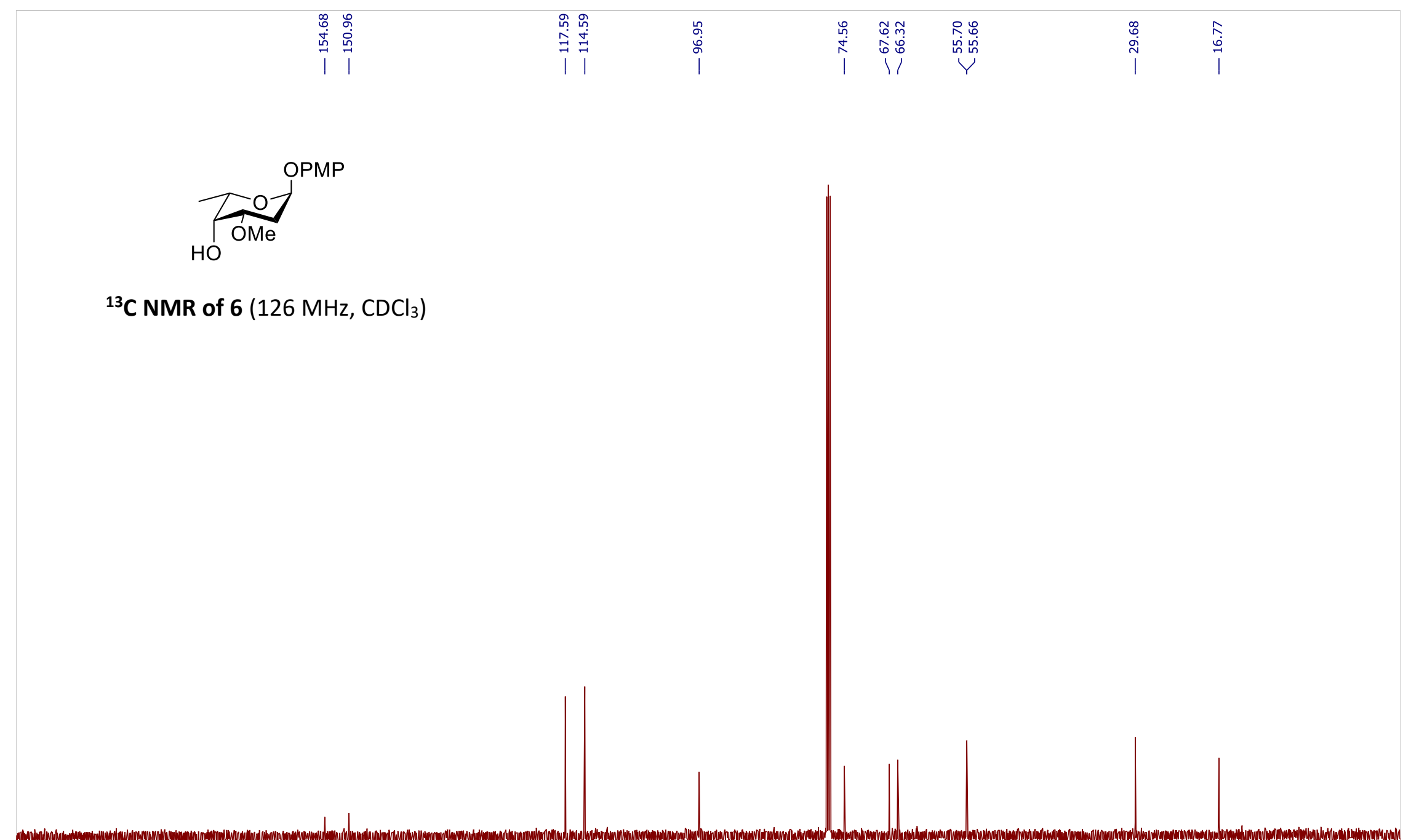

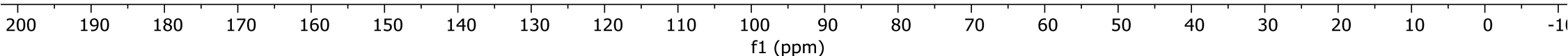




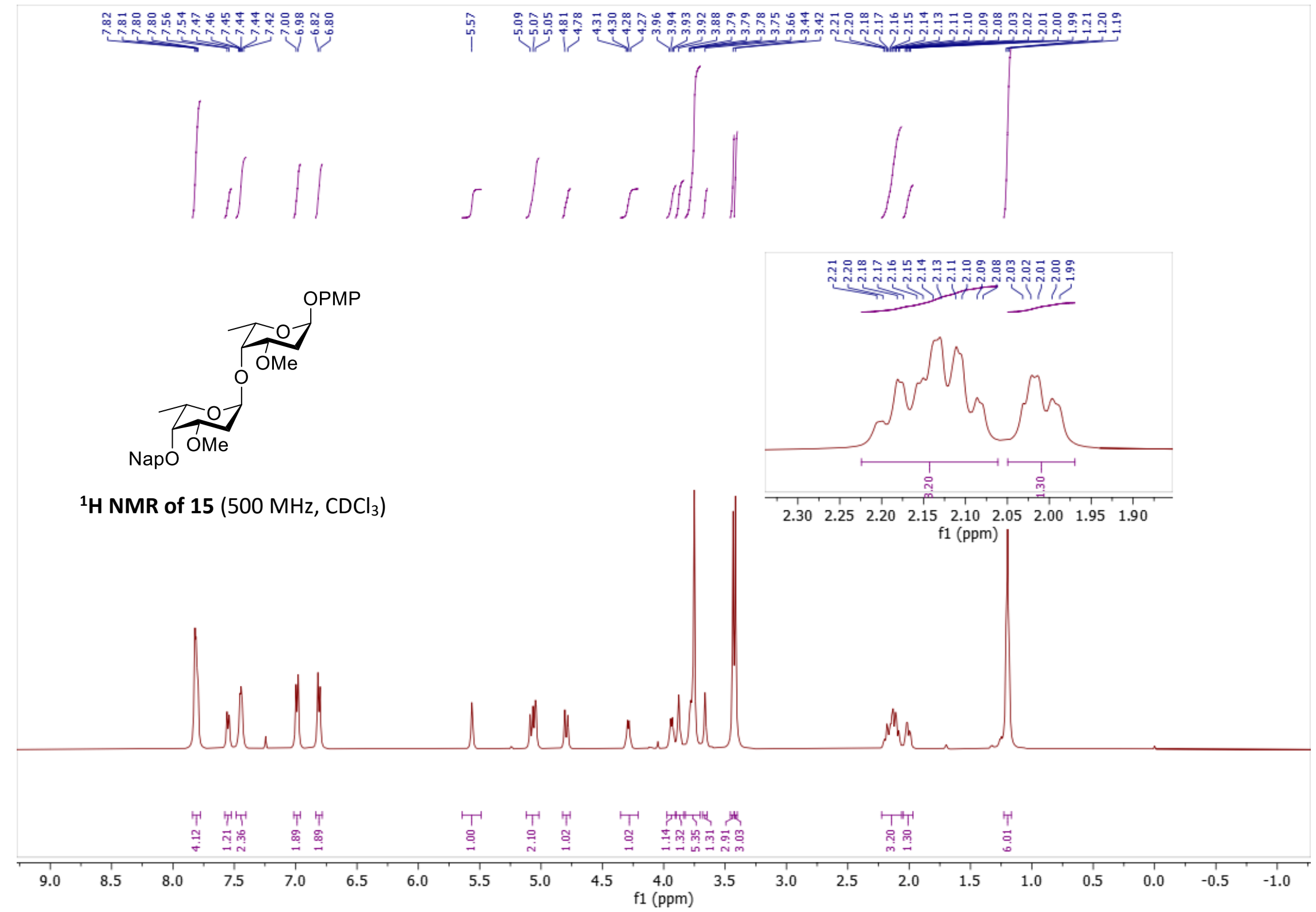




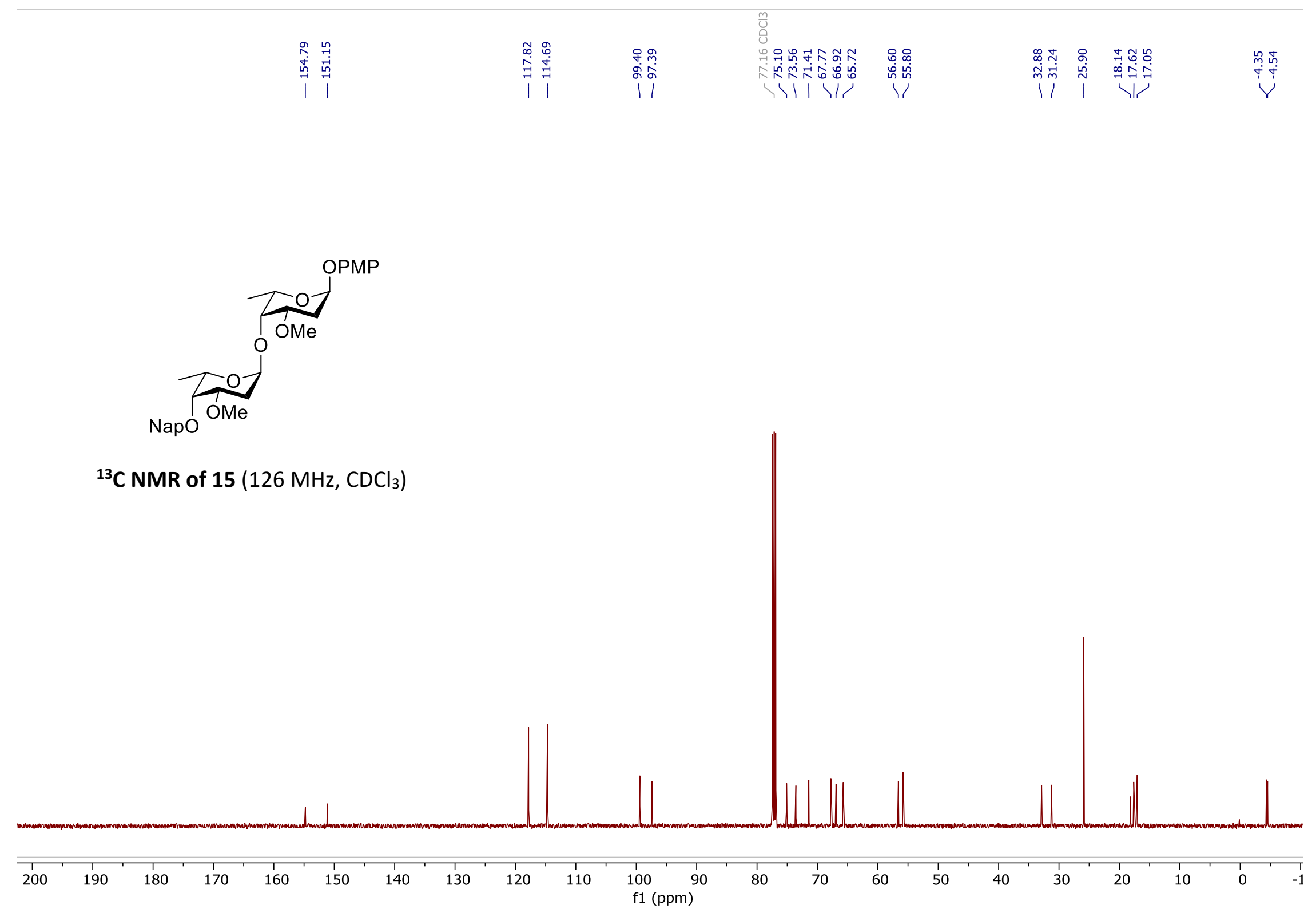




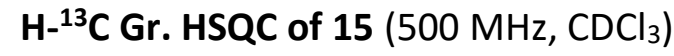

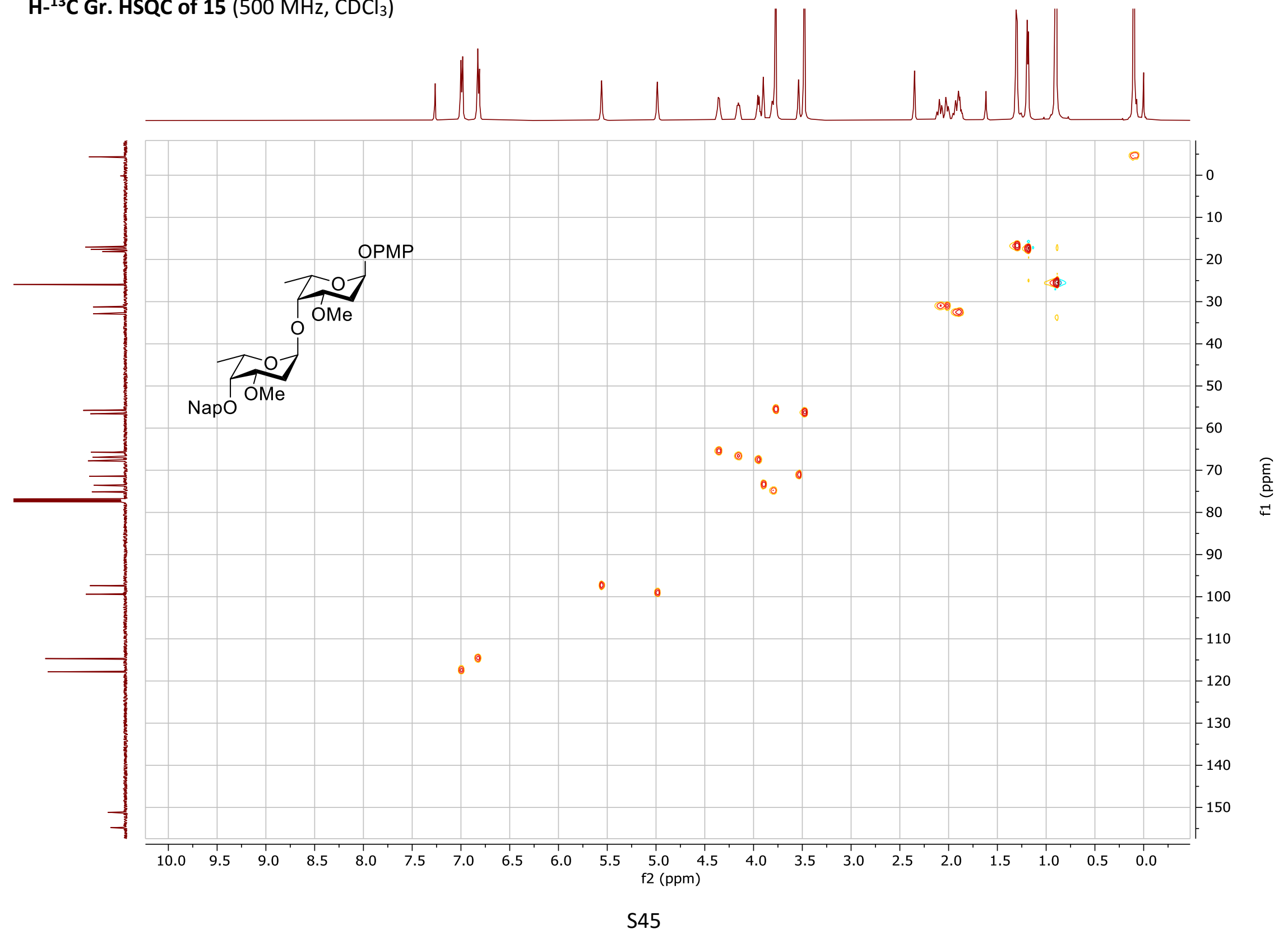


${ }^{1} \mathrm{H}-{ }^{1} \mathrm{H}$ Gr. cosY of $15\left(500 \mathrm{MHz}, \mathrm{CDCl}_{3}\right)$

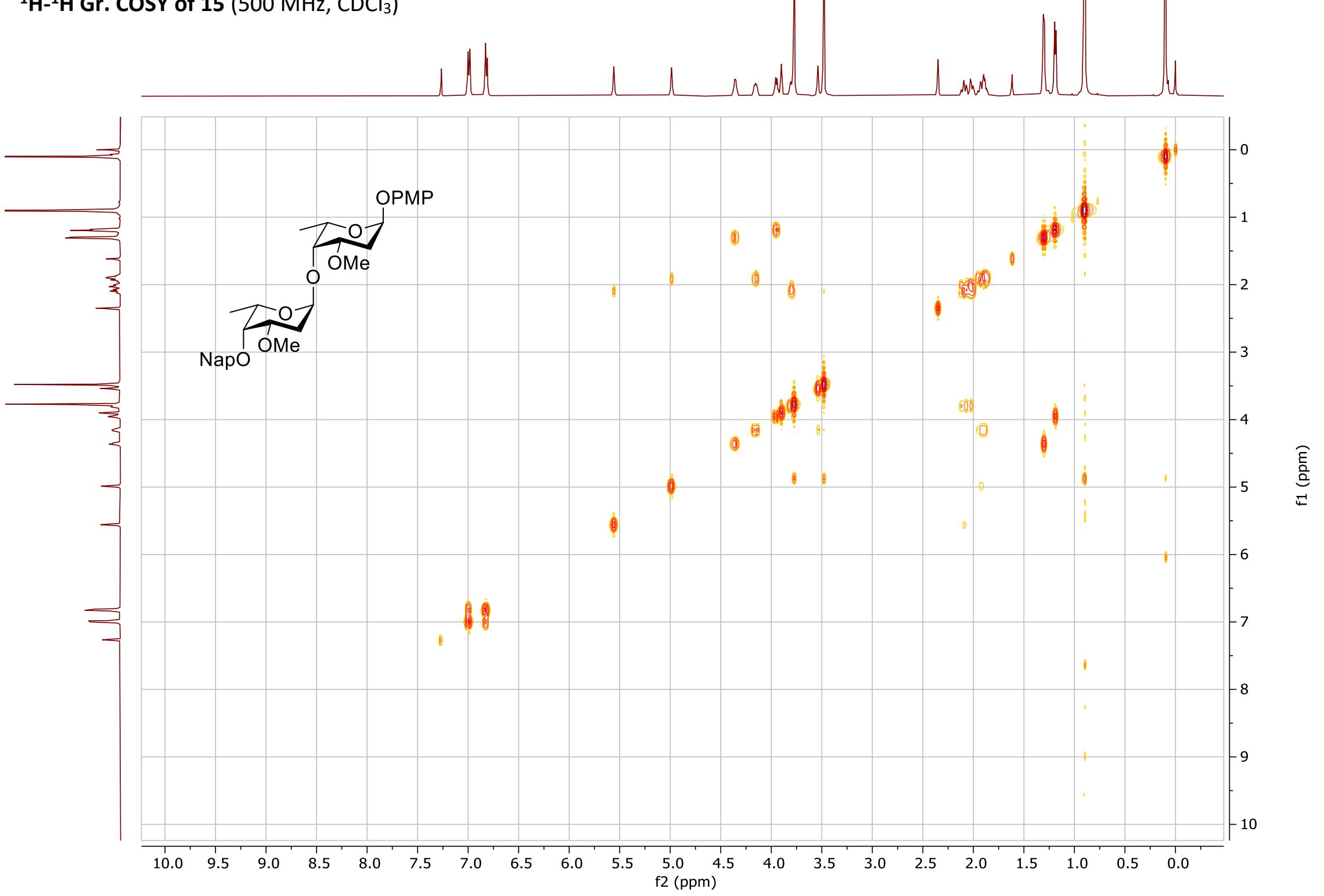




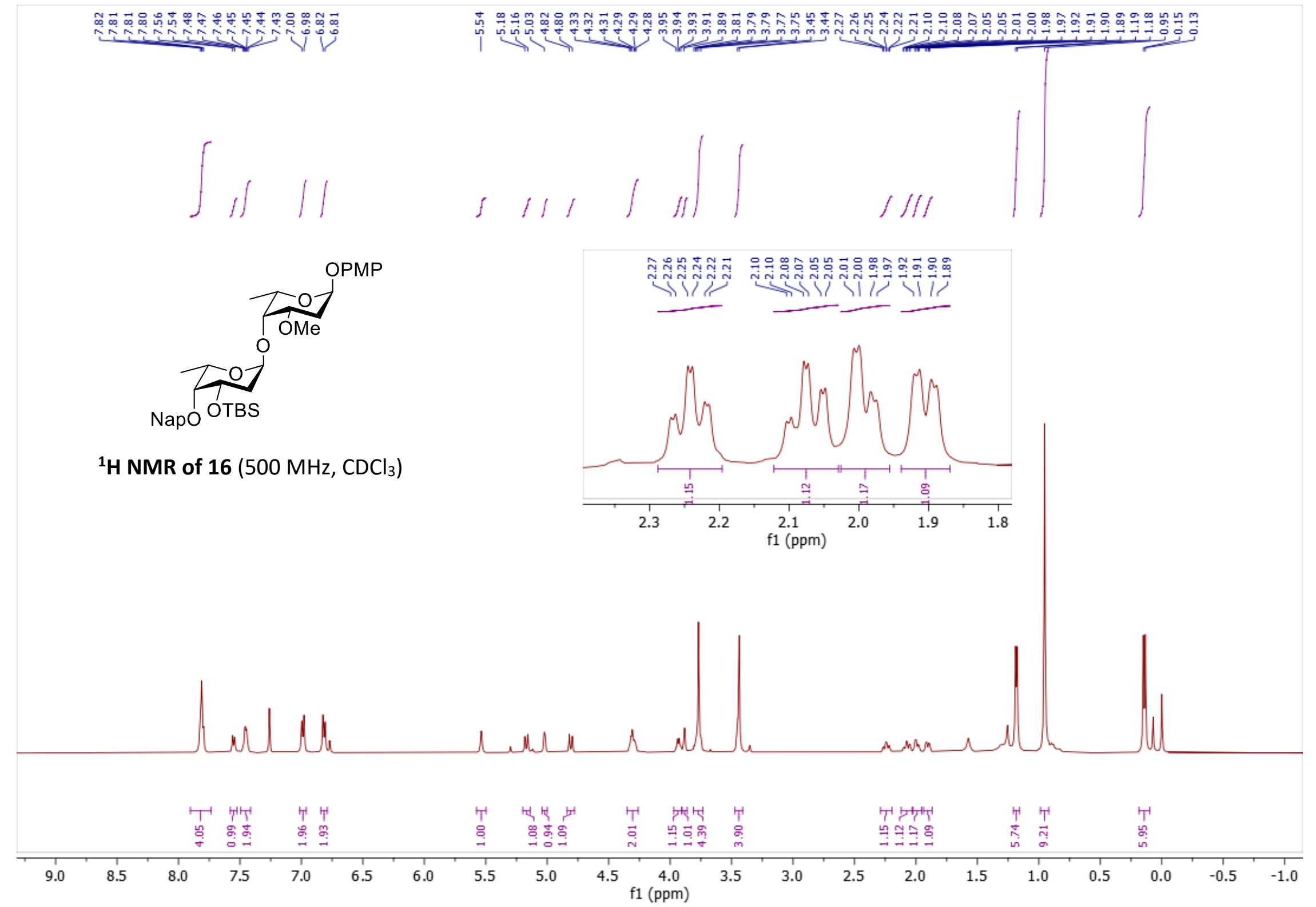




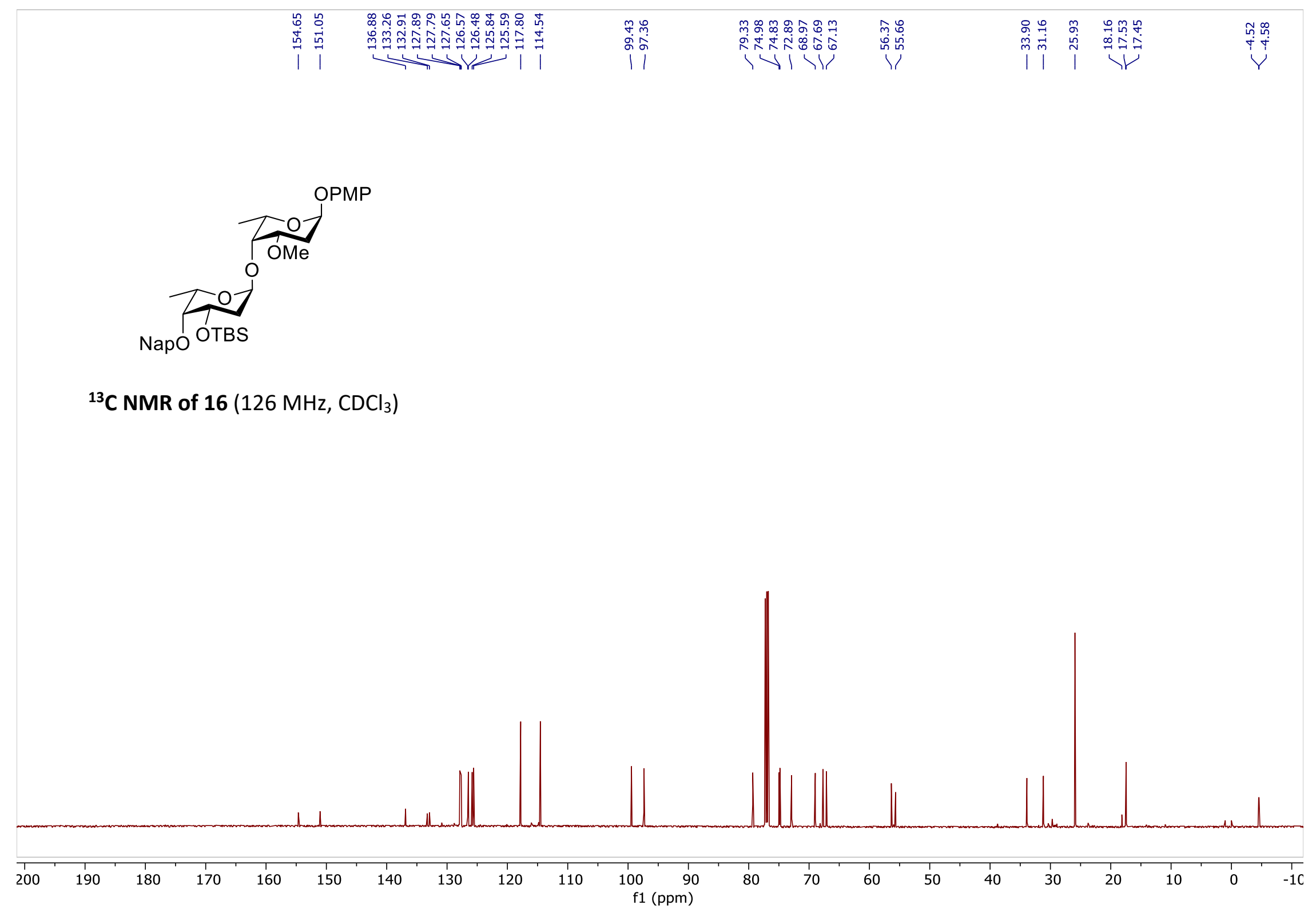


${ }^{H-}{ }^{13} \mathrm{C} \mathrm{Gr}$. HSQC of $16\left(500 \mathrm{MHz}, \mathrm{CDCl}_{3}\right)$

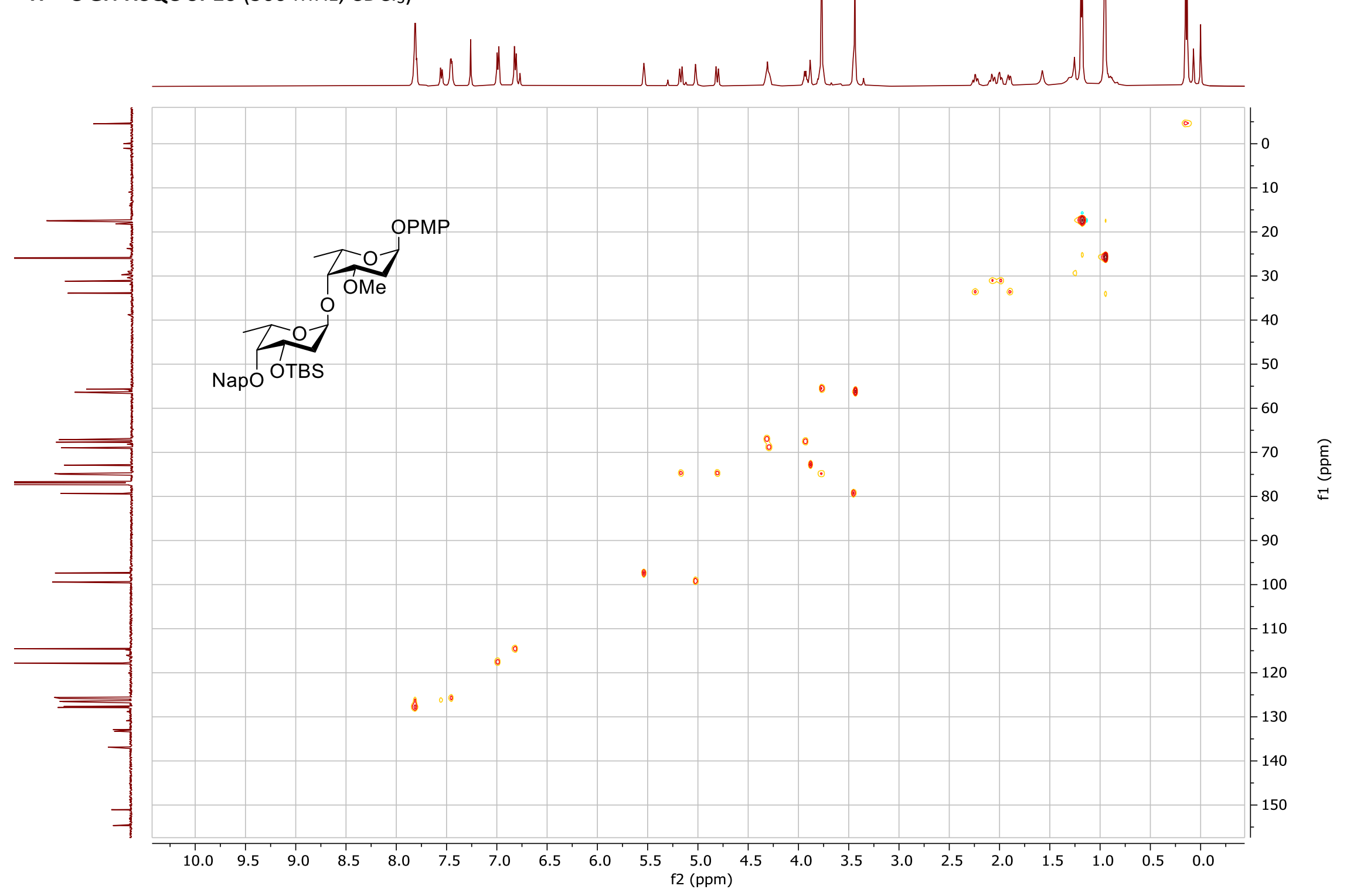


${ }^{1} \mathrm{H}-{ }^{1} \mathrm{H}$ Gr. cosY of $16\left(500 \mathrm{MHz}, \mathrm{CDCl}_{3}\right)$

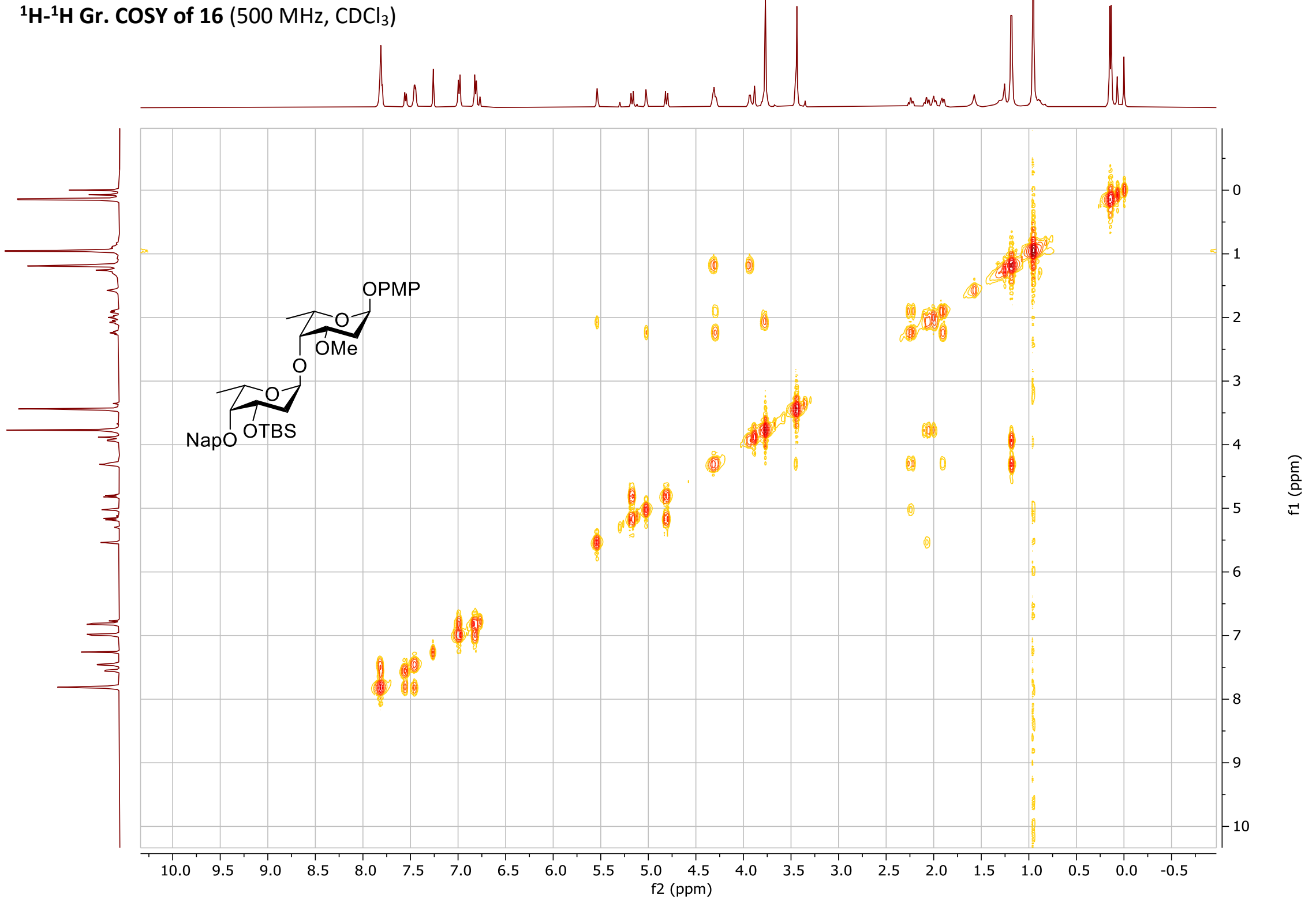




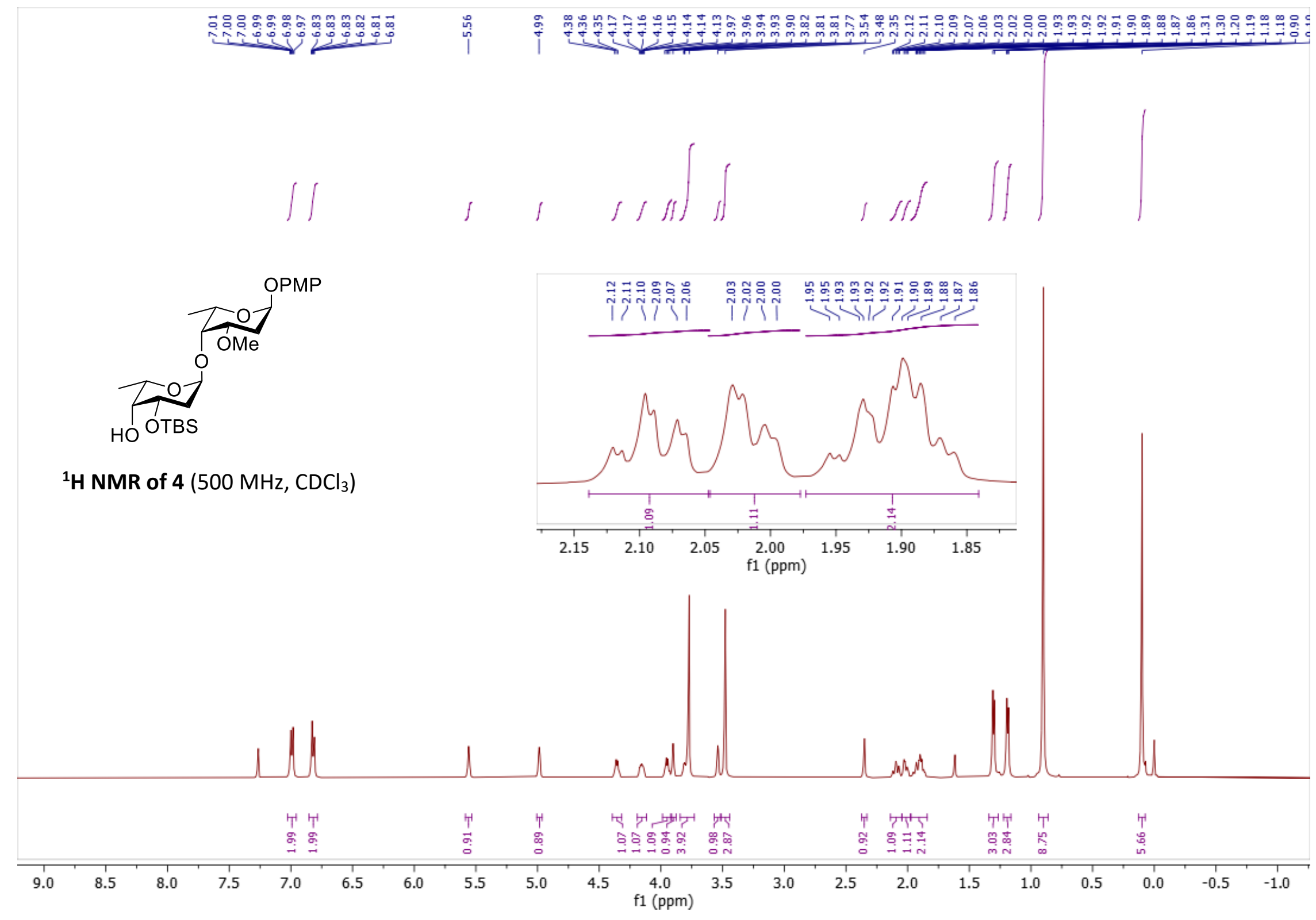




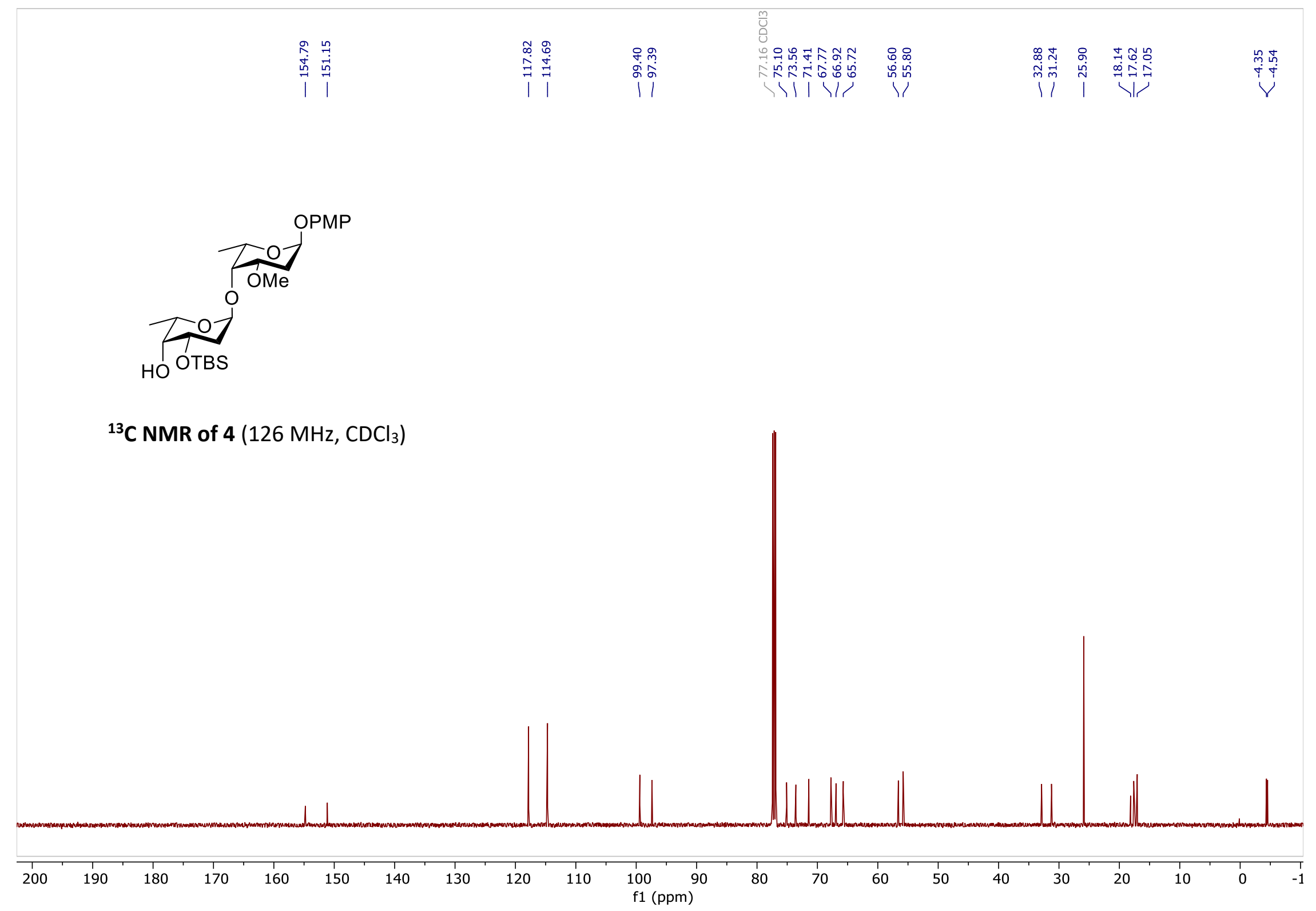


${ }^{H-13} \mathrm{C} \mathrm{Gr}$. HSQC of $4\left(500 \mathrm{MHz}, \mathrm{CDCl}_{3}\right)$
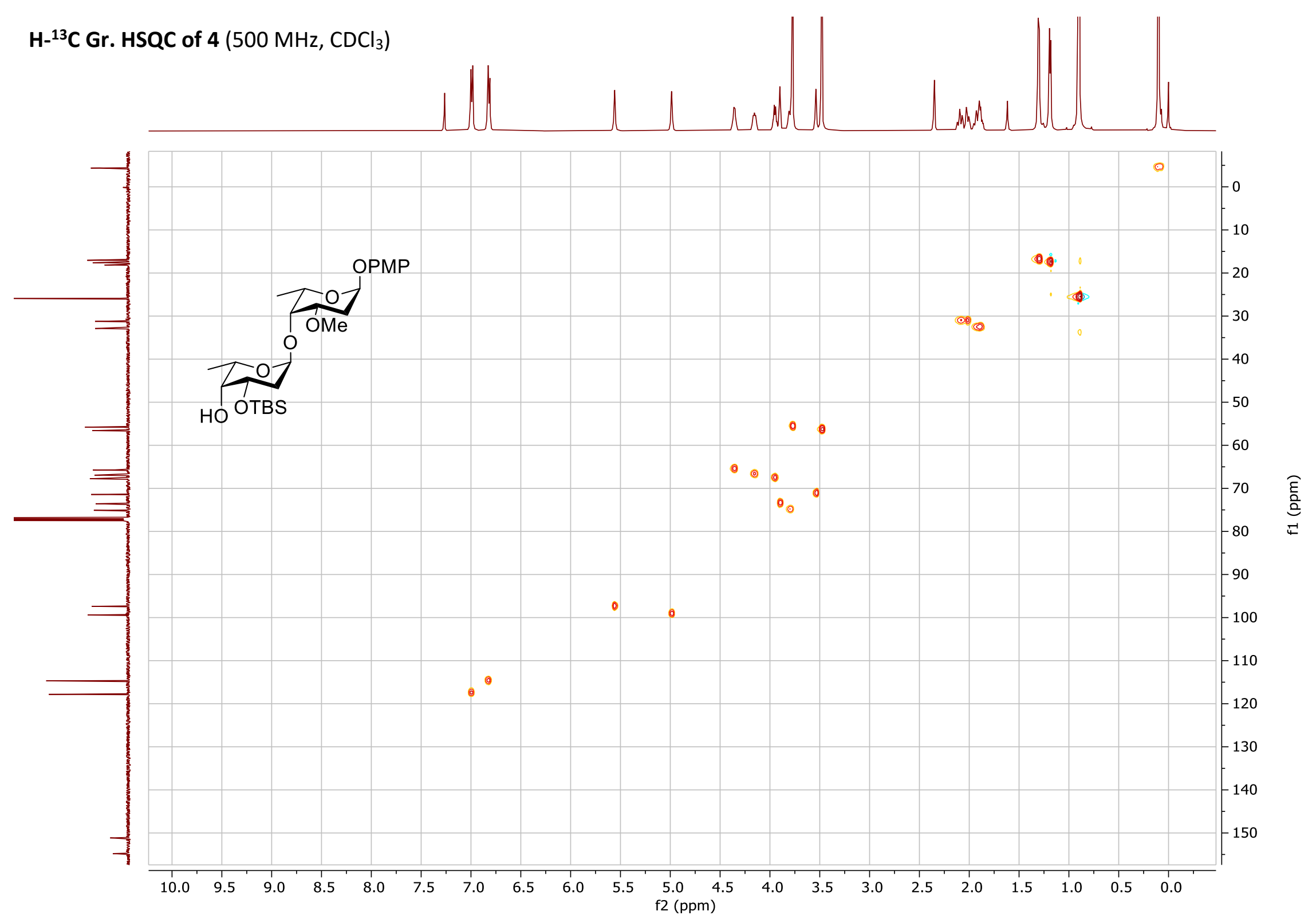
${ }^{1} \mathrm{H}-{ }^{1} \mathrm{H}$ Gr. cosY of $4\left(500 \mathrm{MHz}, \mathrm{CDCl}_{3}\right)$

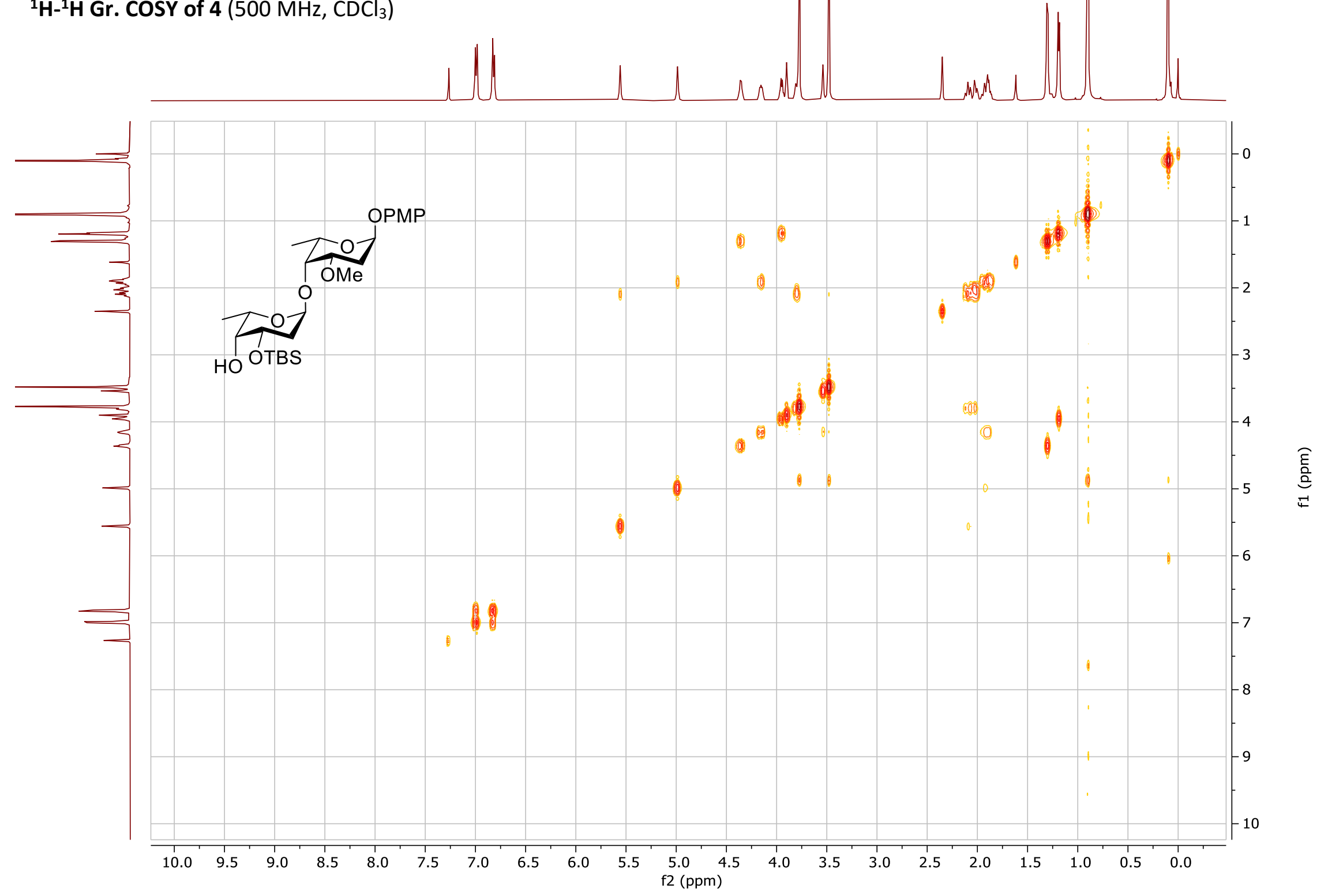


$\frac{m}{\mathrm{C}}$

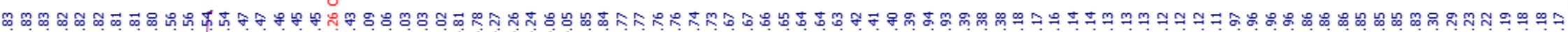

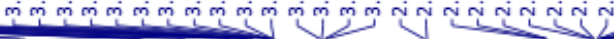
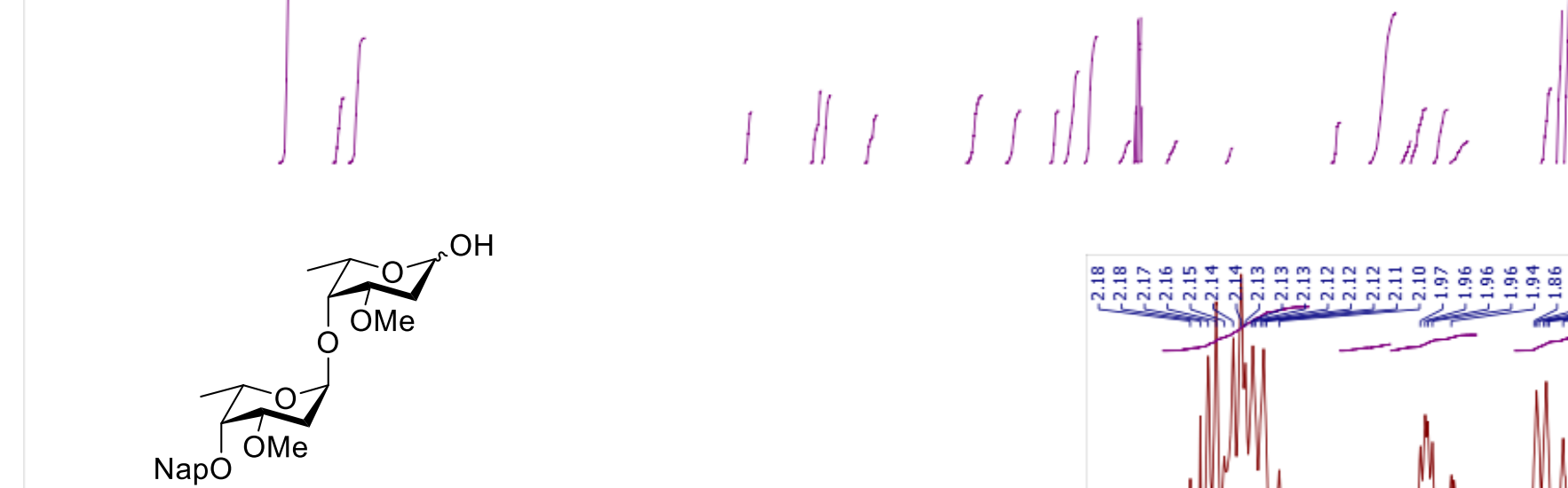

${ }^{1} \mathbf{H}$ NMR of $5(500 \mathrm{MHz}, \mathrm{CDCl} 3)$

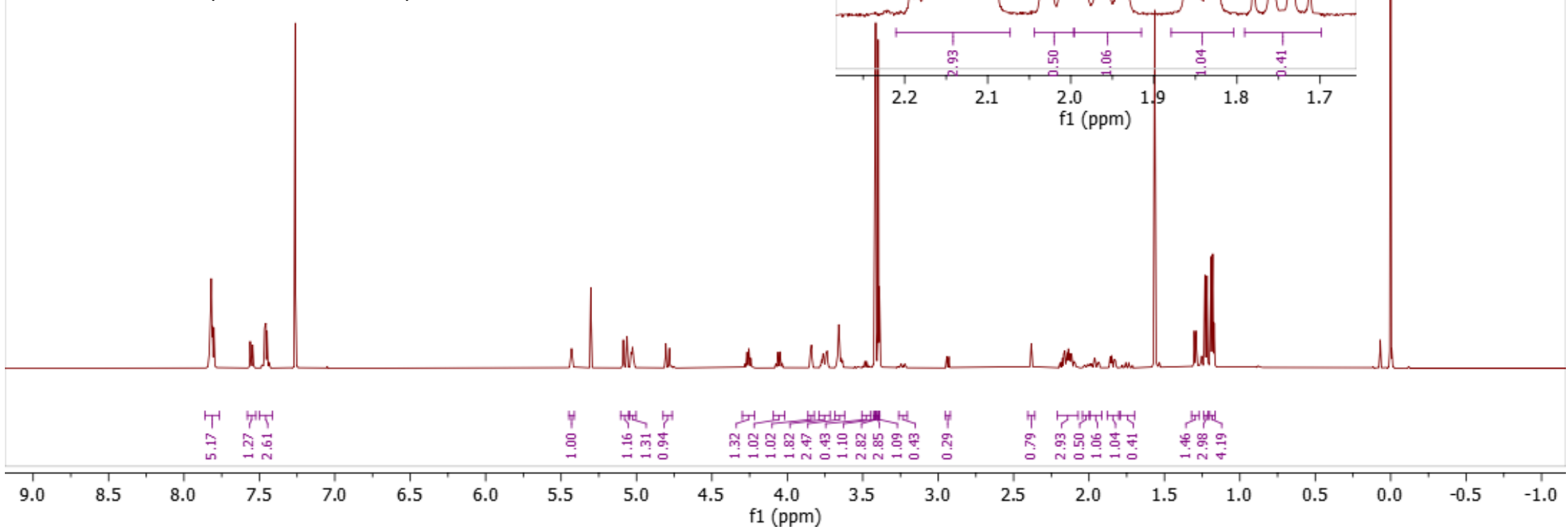




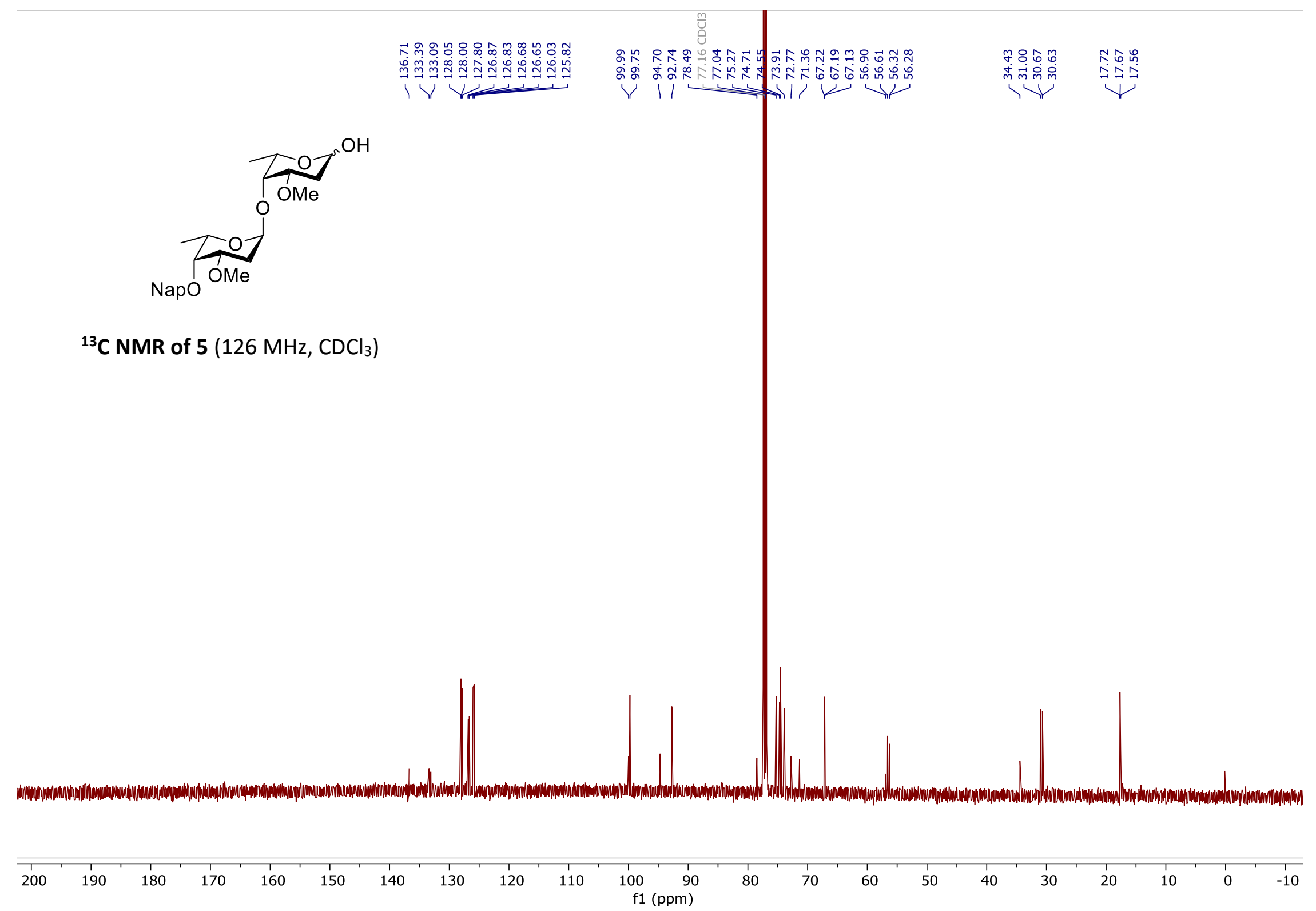


H- ${ }^{13} \mathrm{C} \mathrm{Gr}$. HSQC of 5 (500 MHz, $\left.\mathrm{CDCl}_{3}\right)$
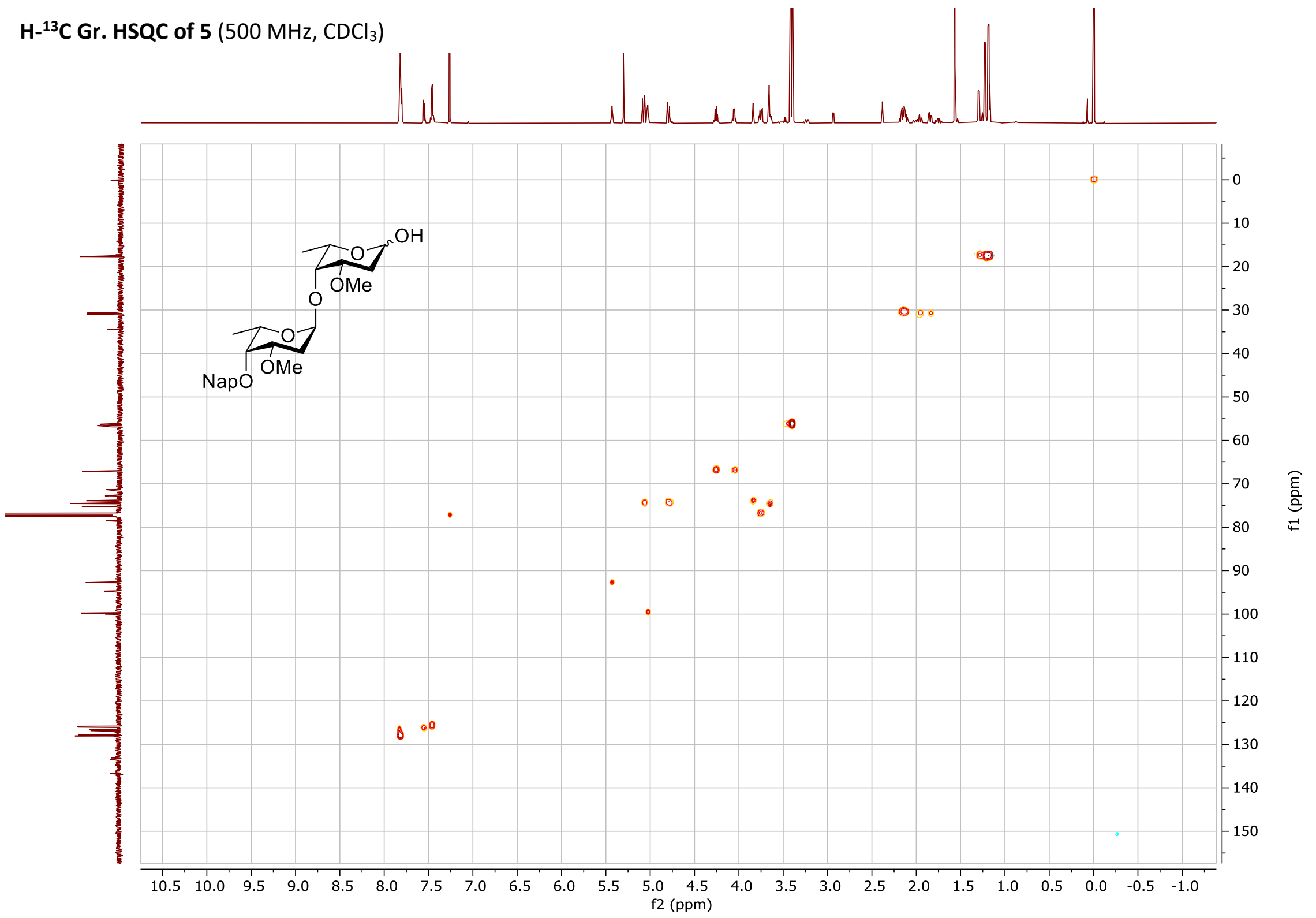
${ }^{1} \mathbf{H}-{ }^{1} \mathbf{H}$ Gr. COSY of $5\left(500 \mathrm{MHz}, \mathrm{CDCl}_{3}\right)$
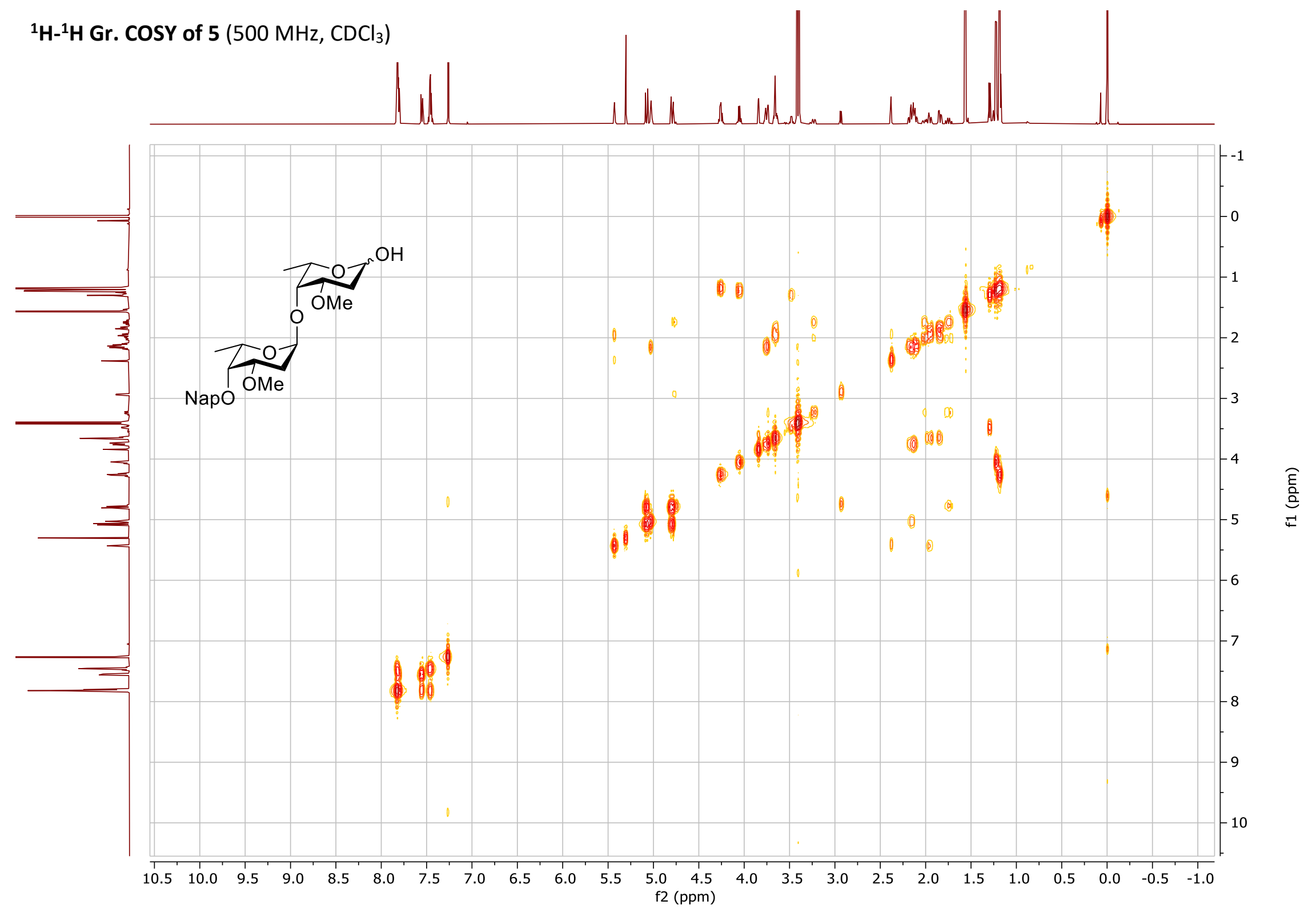


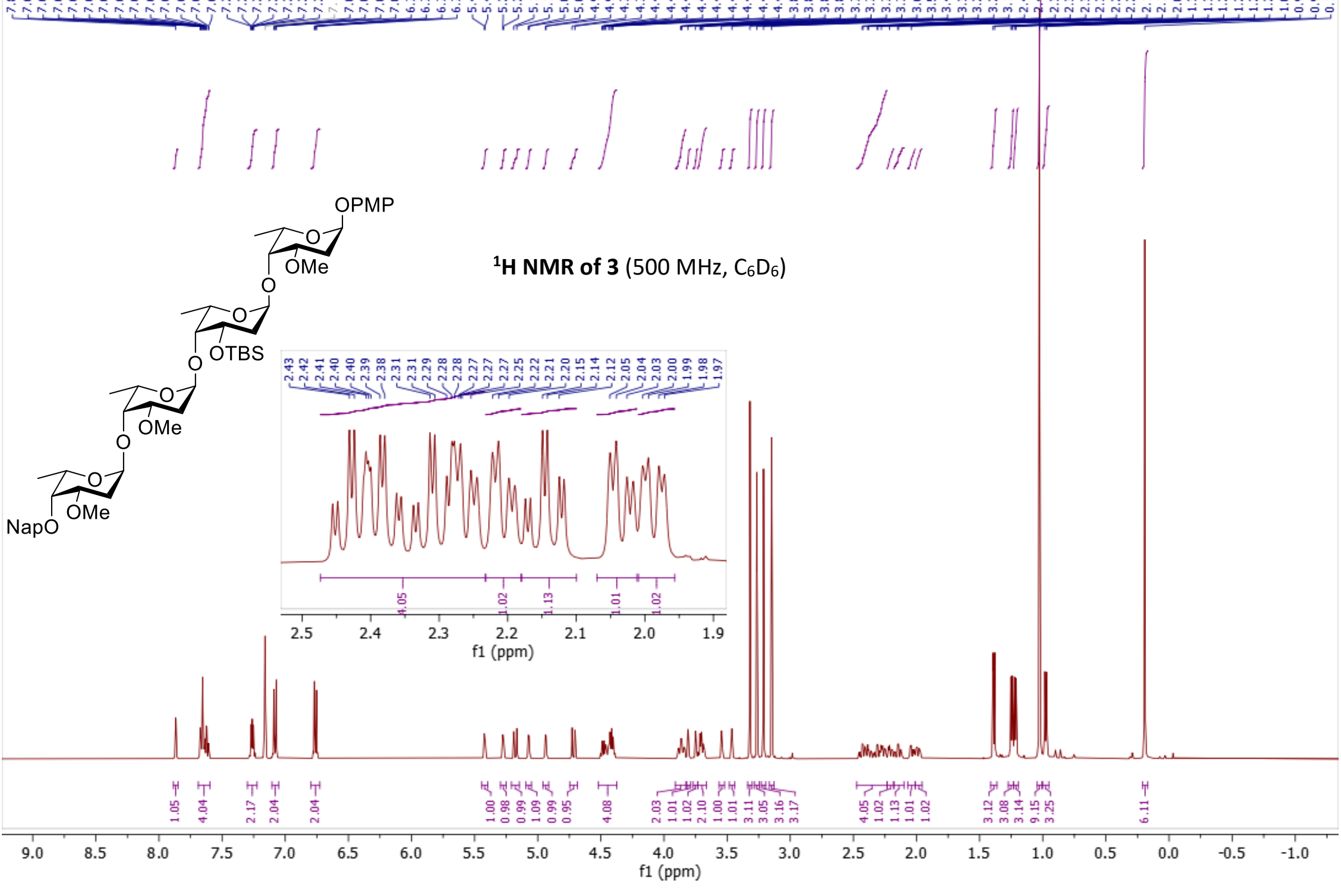




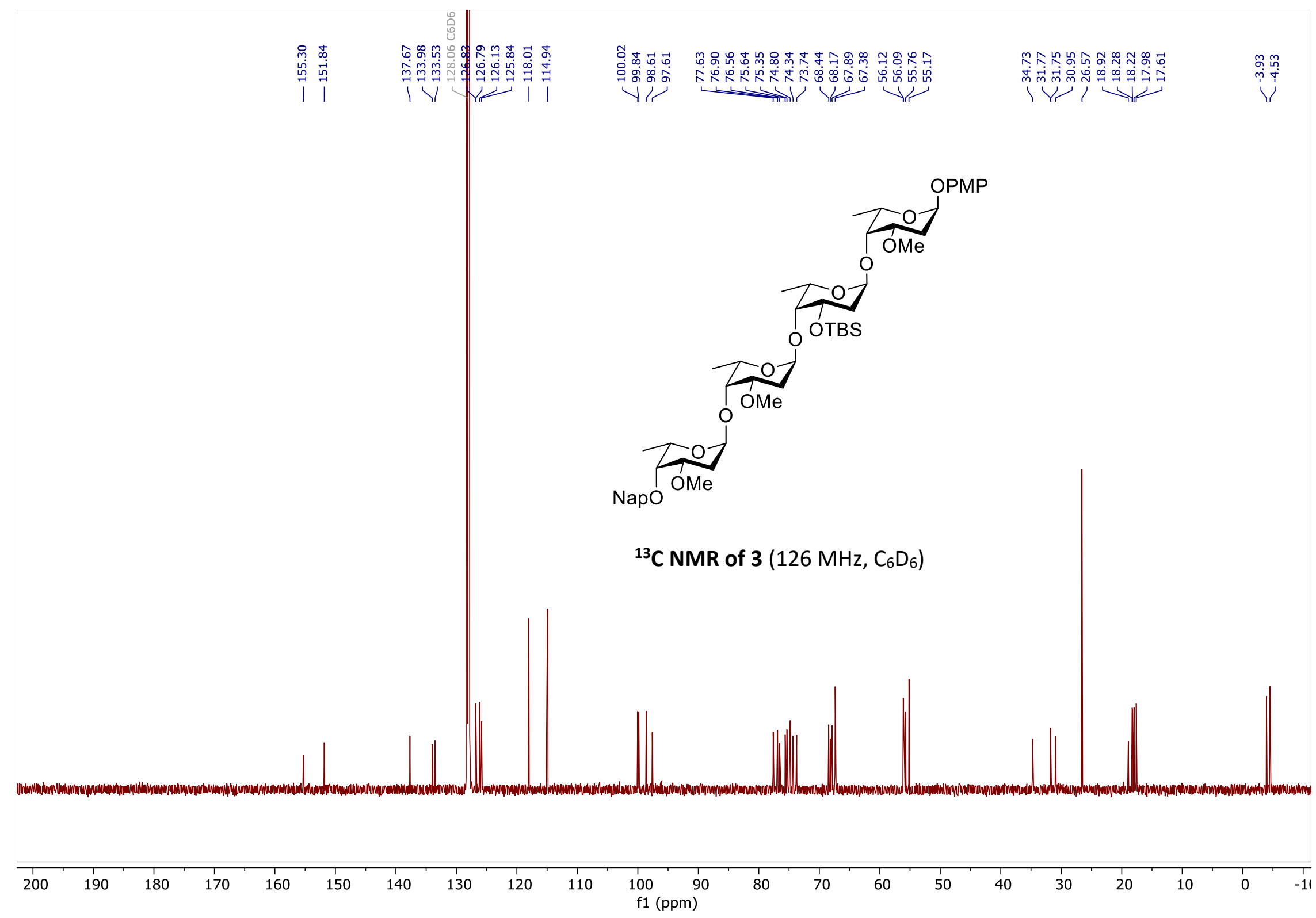


${ }^{H-}{ }^{13} \mathrm{C}$ Gr. HSQC of $3\left(500 \mathrm{MHz}, \mathrm{C}_{6} \mathrm{D}_{6}\right)$

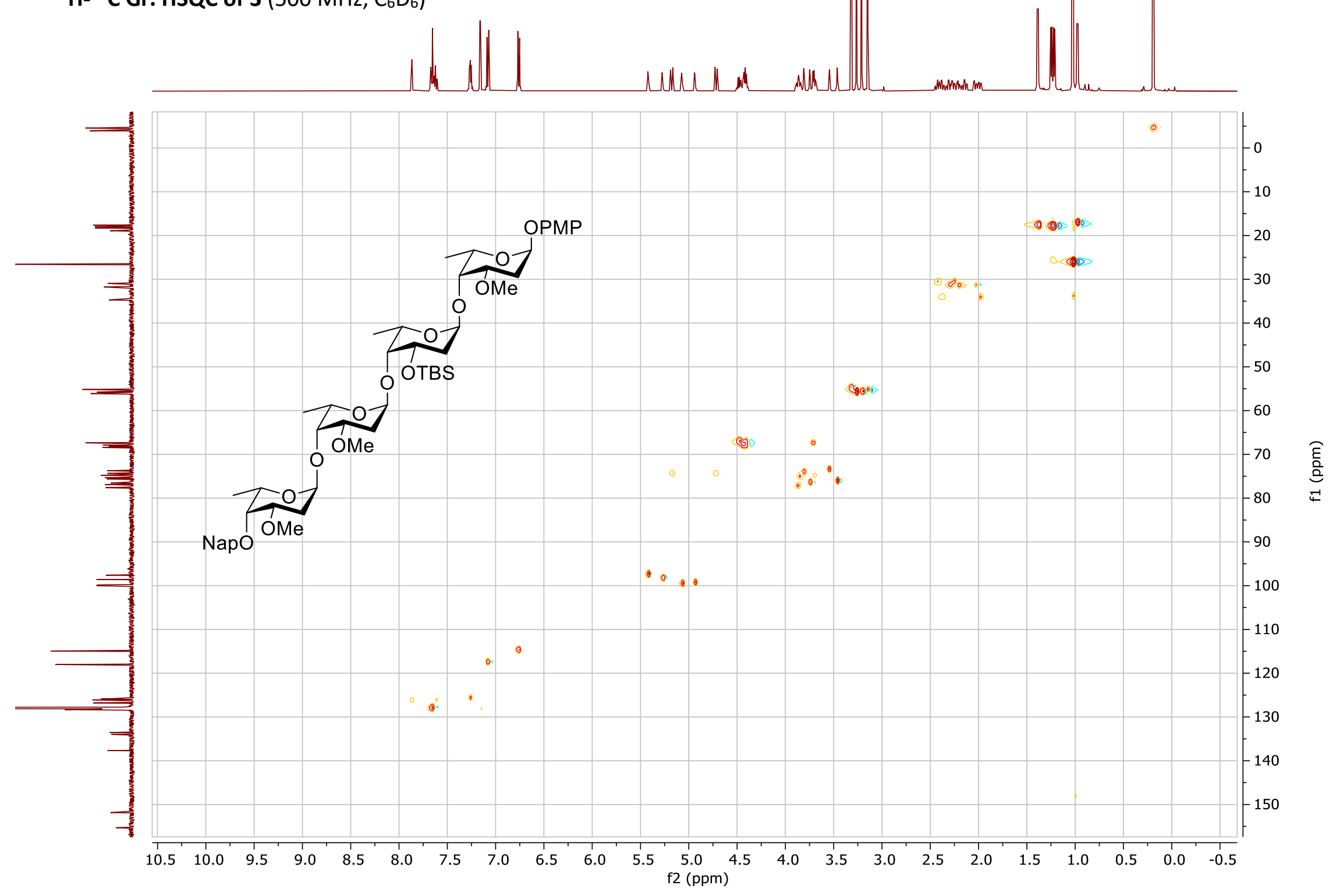


${ }^{1} \mathbf{H}-{ }^{1} \mathbf{H}$ Gr. COSY of $3\left(500 \mathrm{MHz}, \mathrm{C}_{6} \mathrm{D}_{6}\right)$

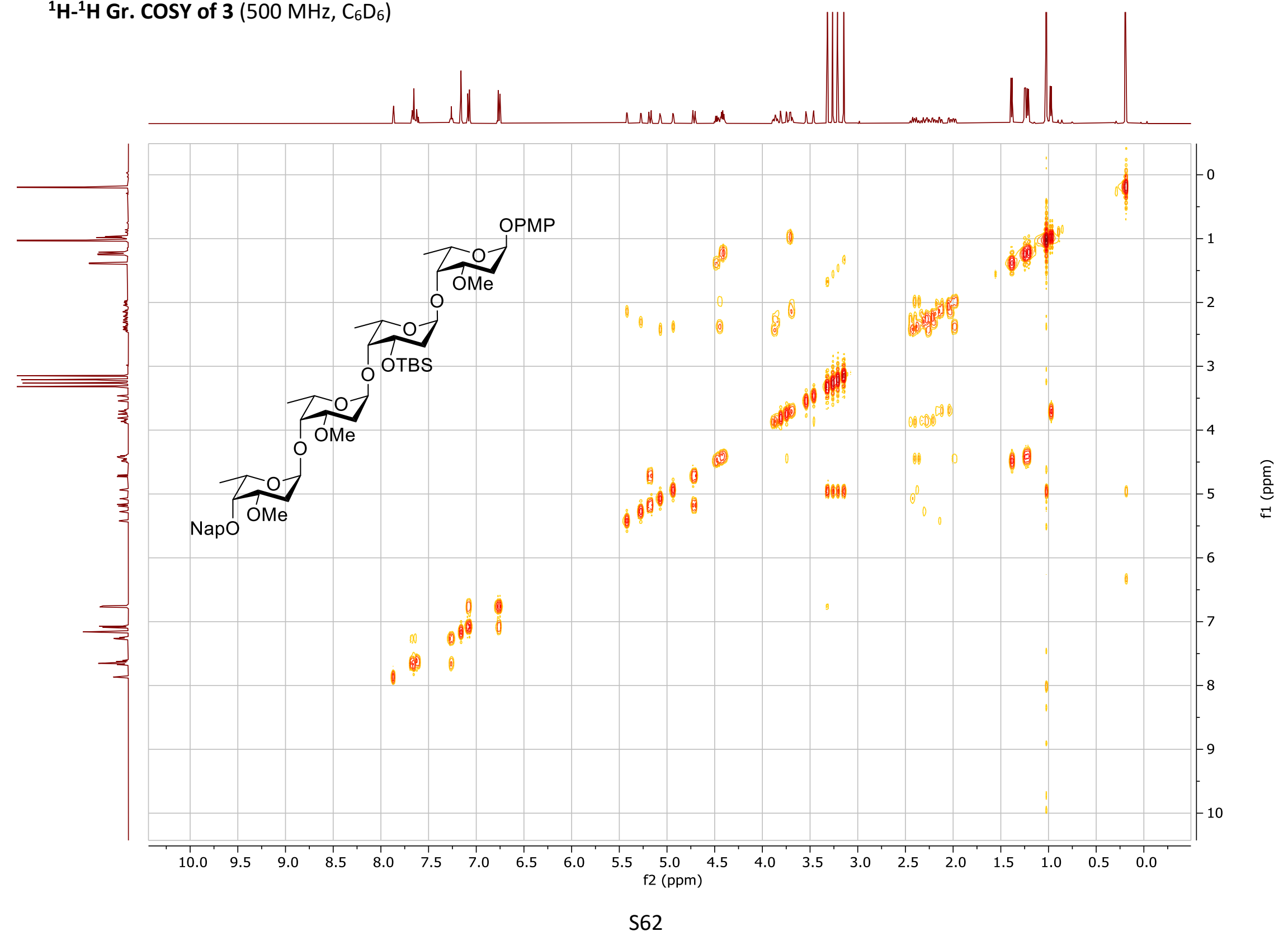




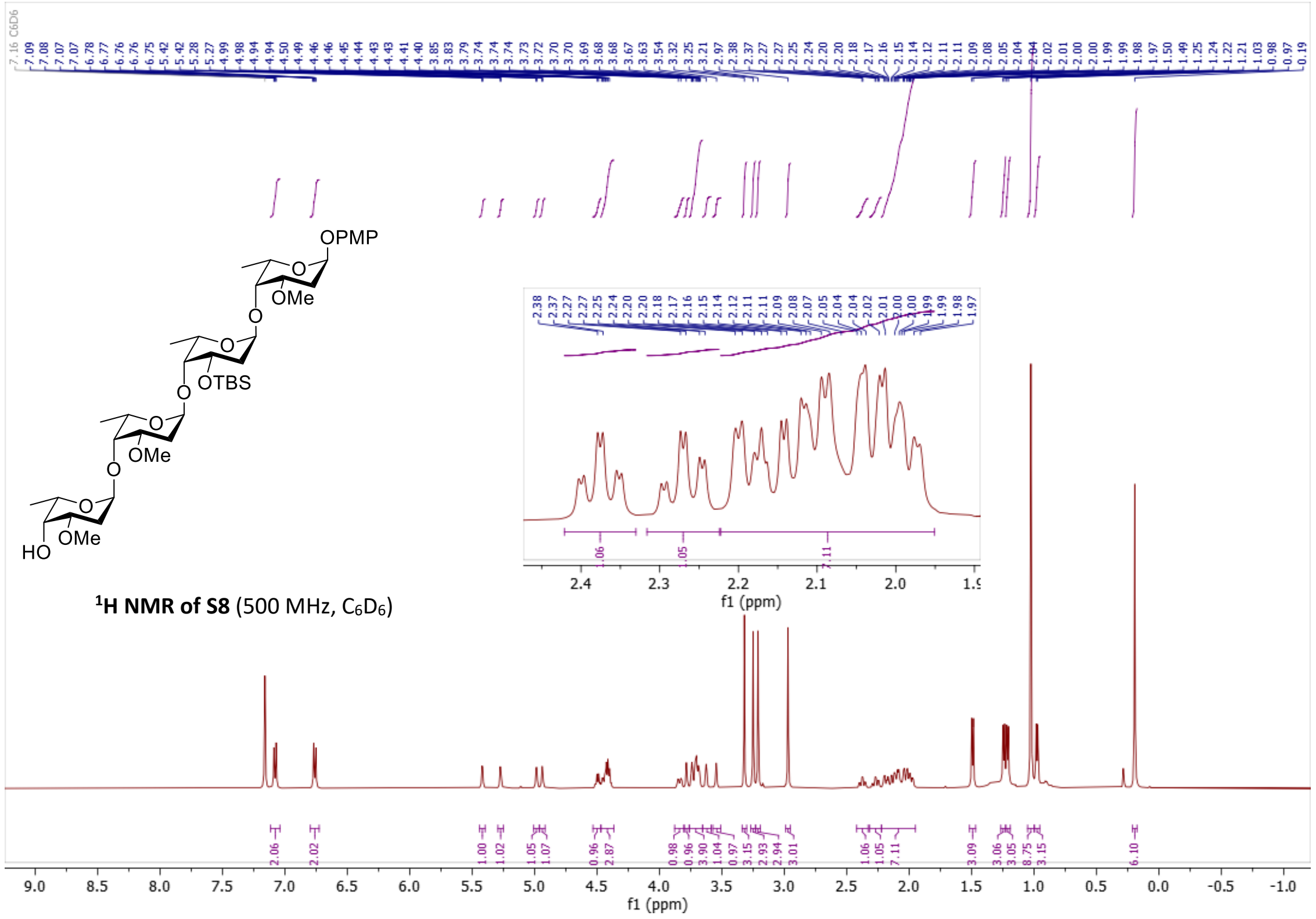




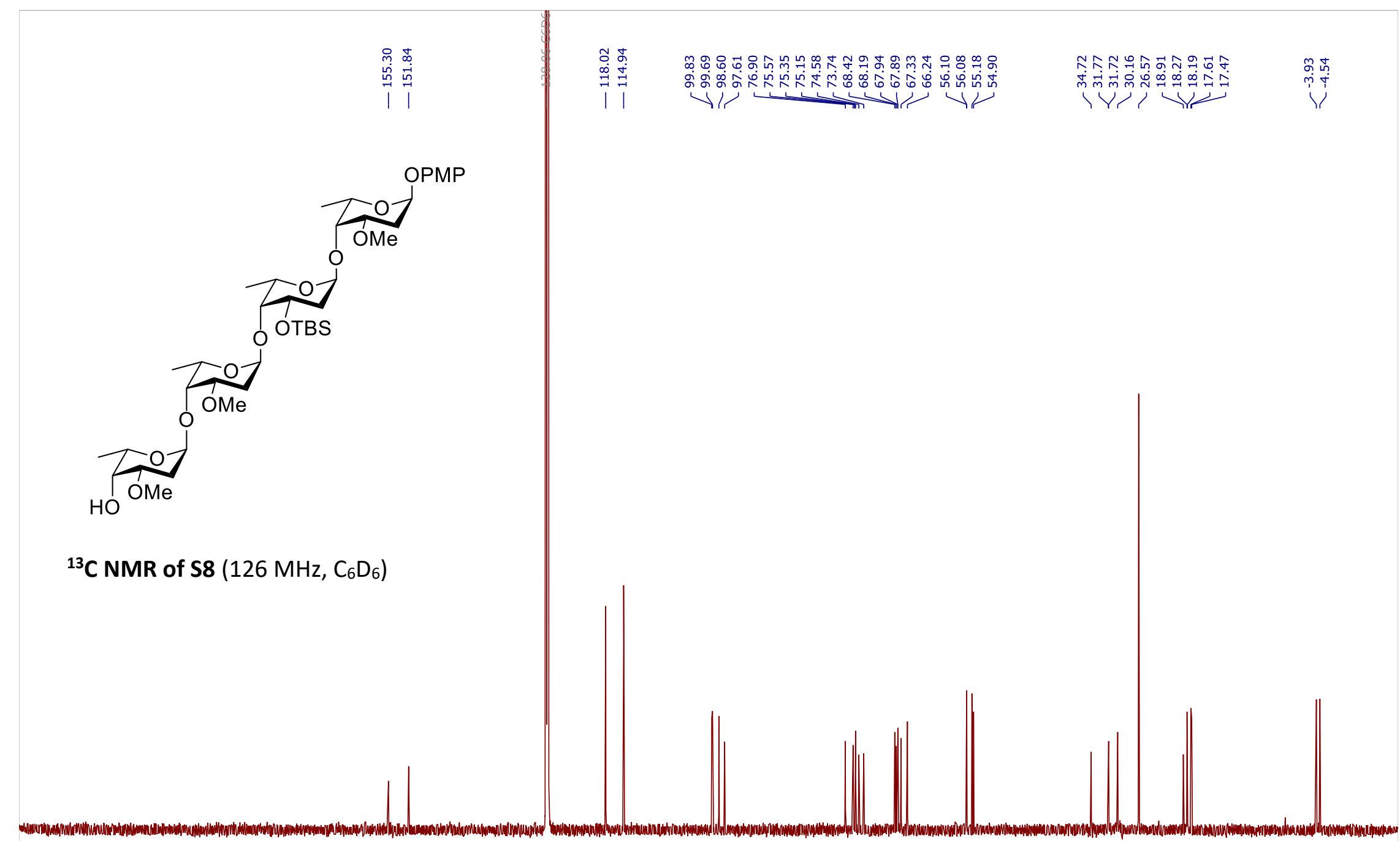

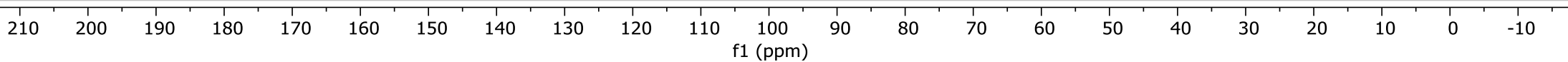


${ }^{H-}{ }^{13} \mathrm{C}$ Gr. HSQC of S8 (500 MHz, $\left.\mathrm{C}_{6} \mathrm{D}_{6}\right)$

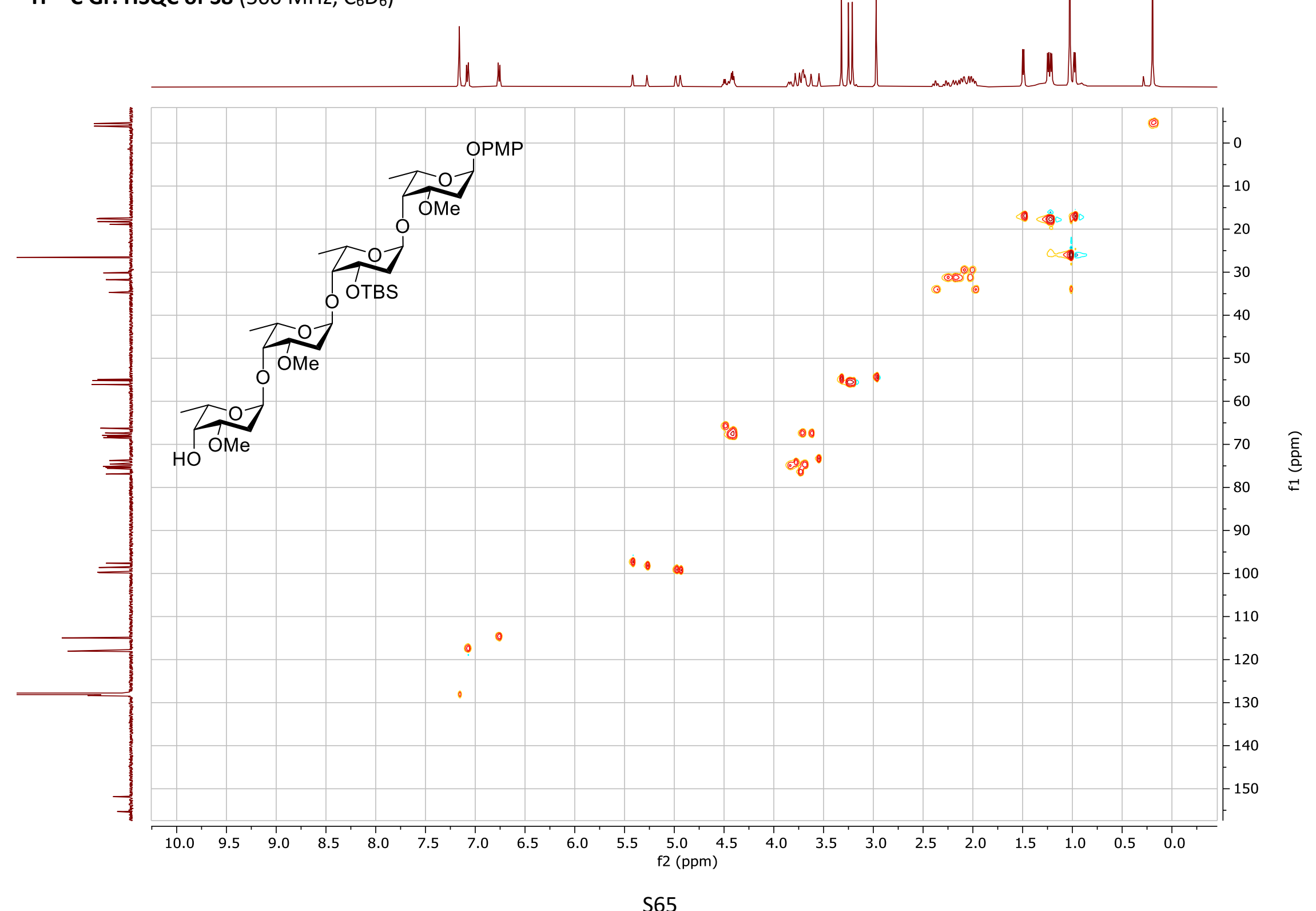


${ }^{1} \mathrm{H}-{ }^{1} \mathrm{H}$ Gr. COSY of S8 (500 MHz, $\left.\mathrm{C}_{6} \mathrm{D}_{6}\right)$

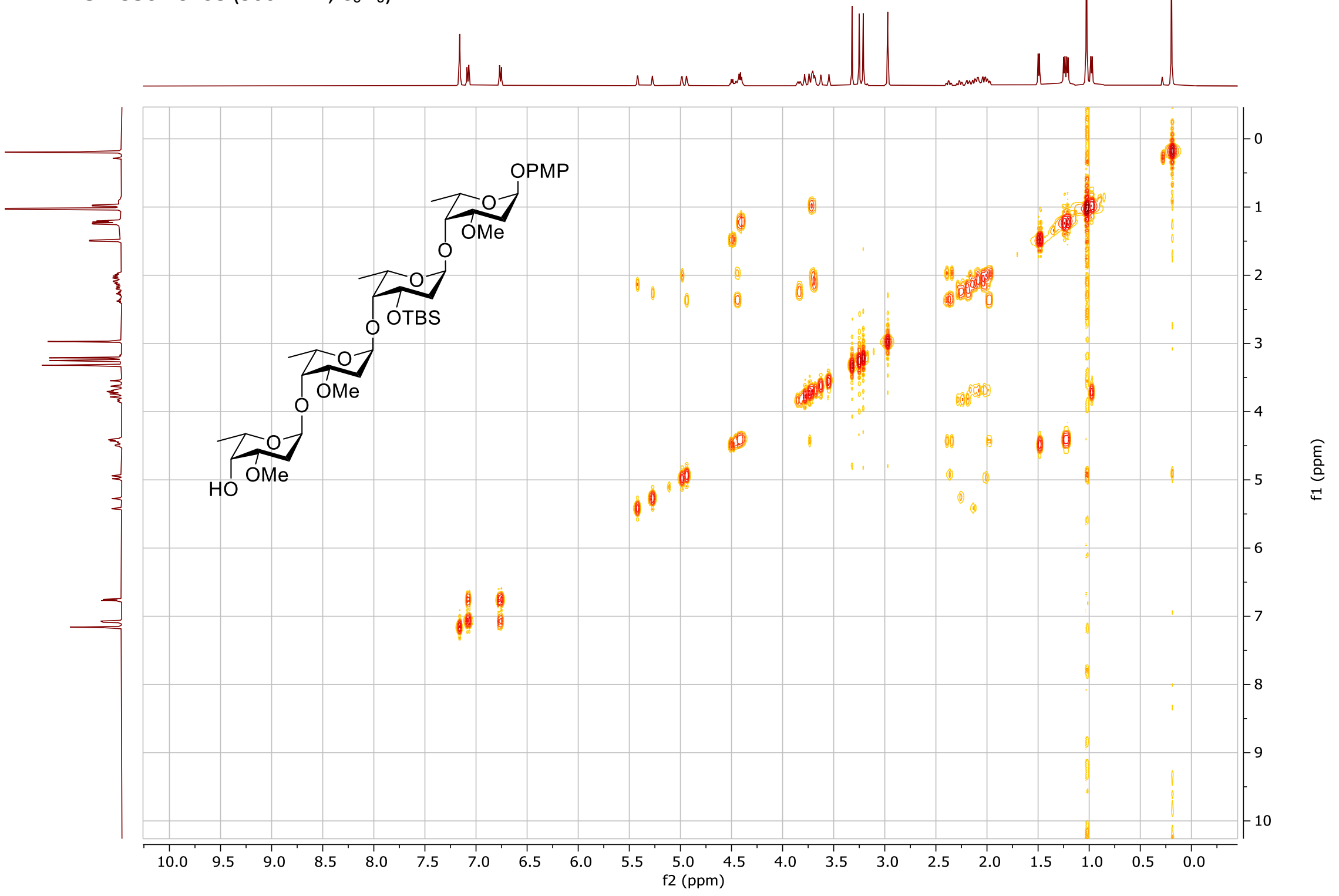




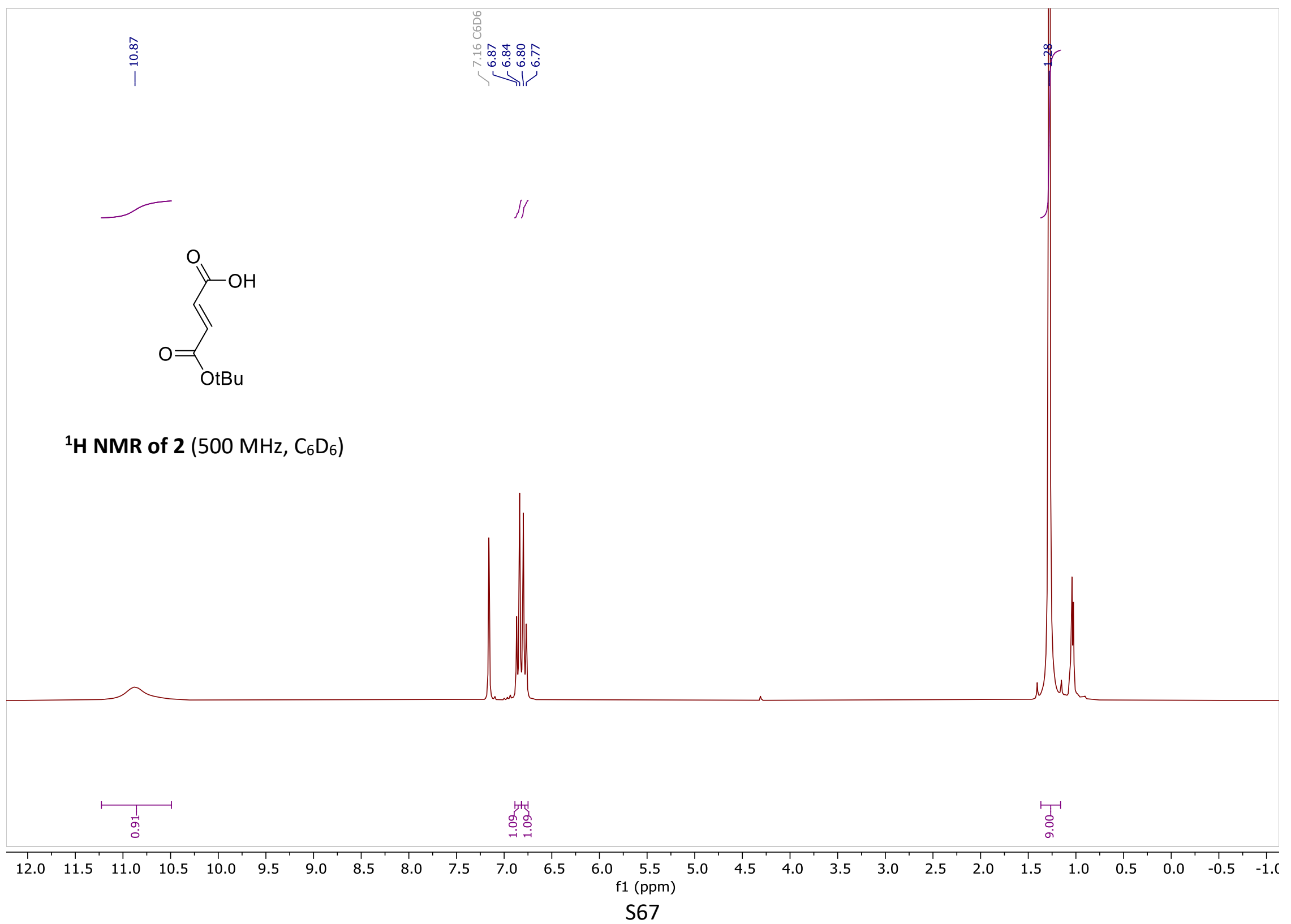




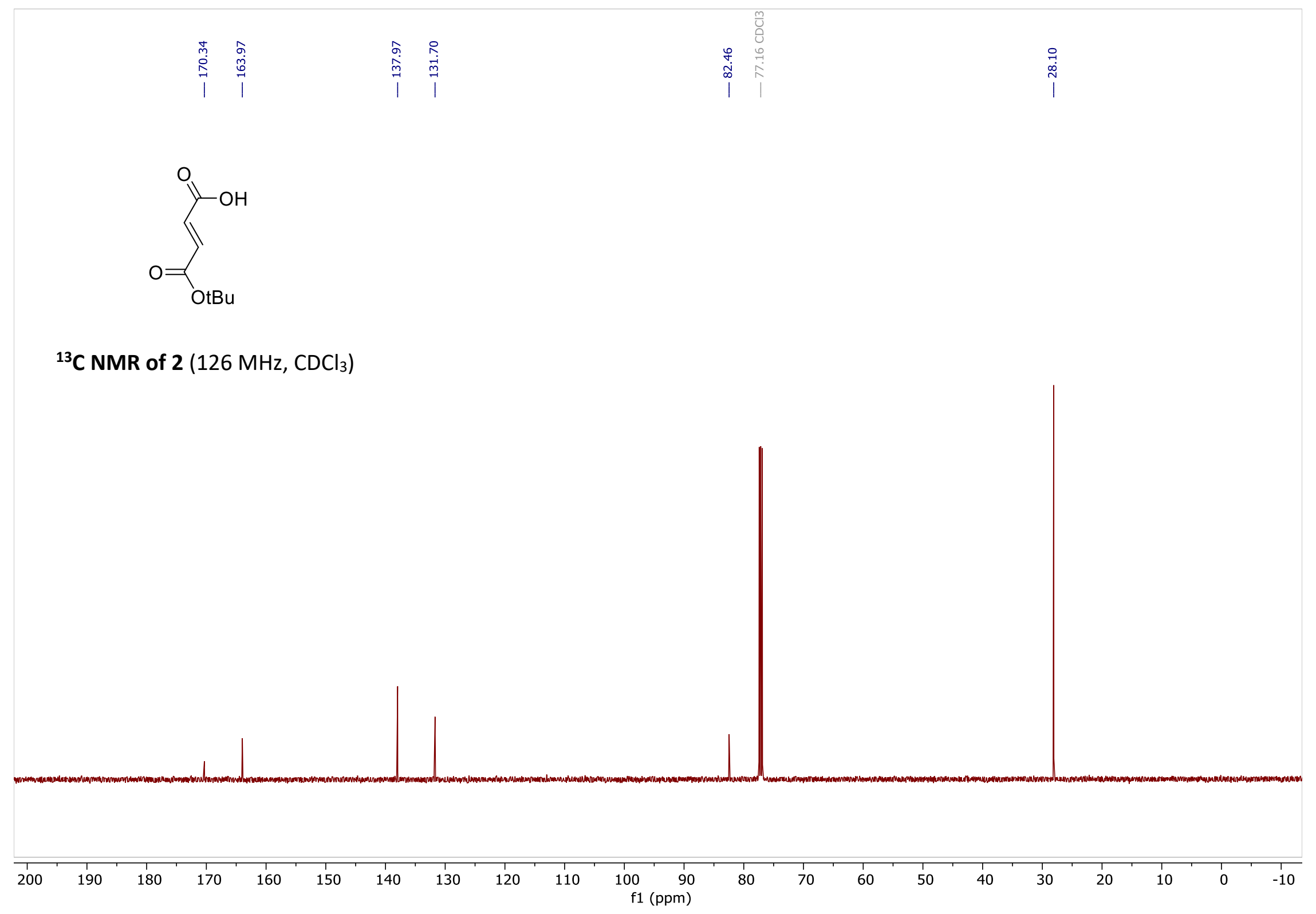




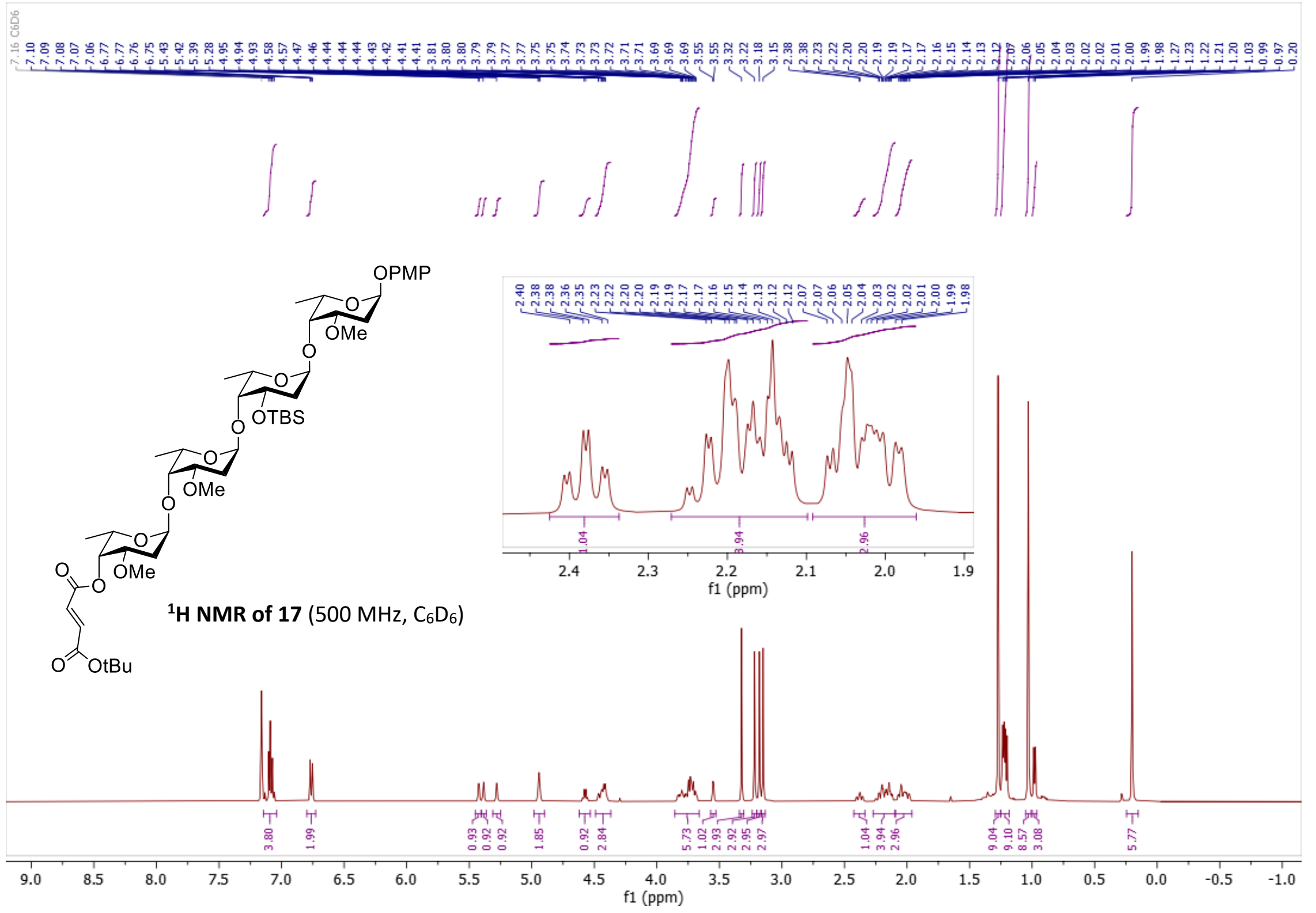




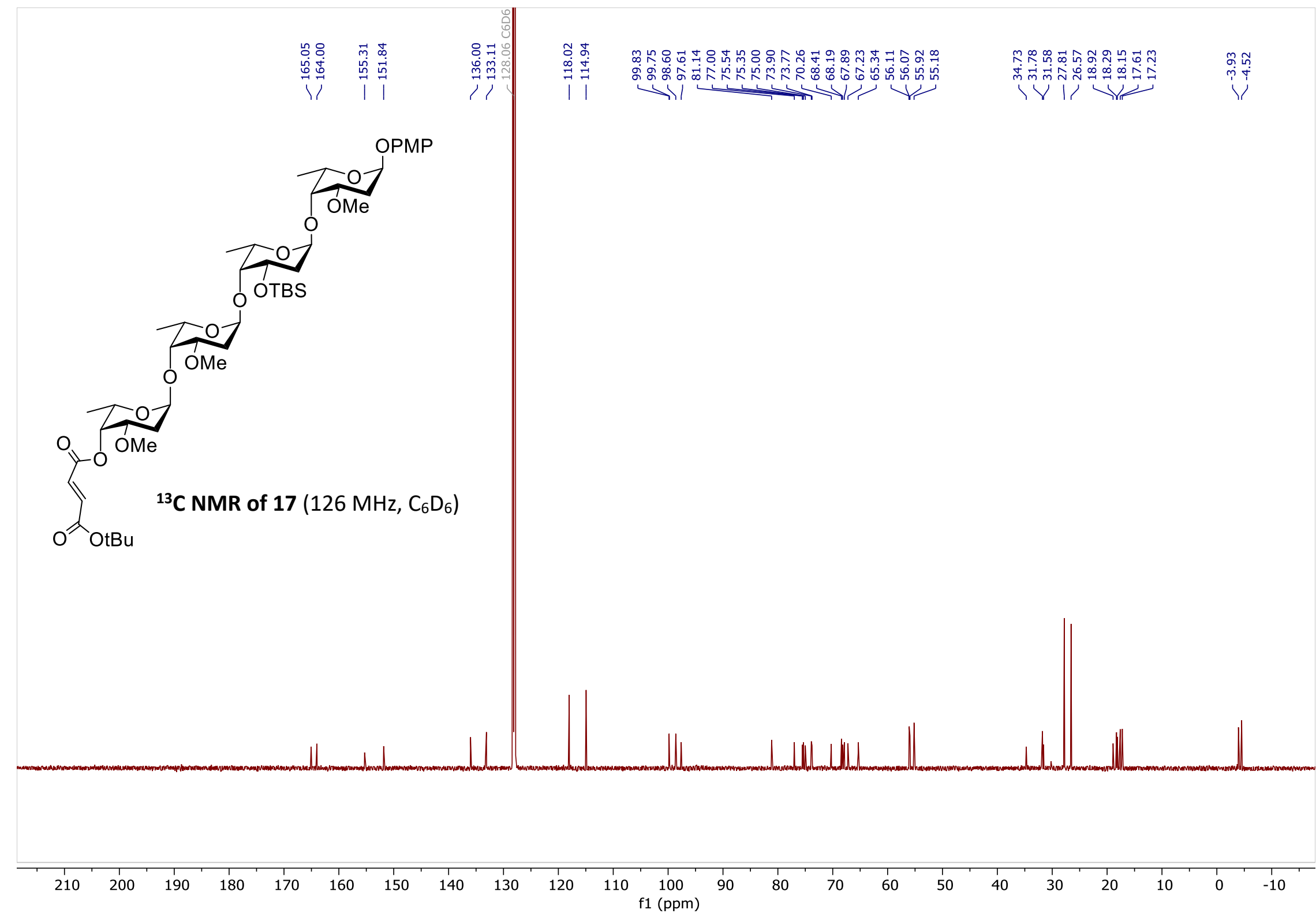


${ }^{H-}{ }^{13} \mathrm{C} \mathrm{Gr}$. HSQC of 17 (500 MHz, $\left.\mathrm{C}_{6} \mathrm{D}_{6}\right)$
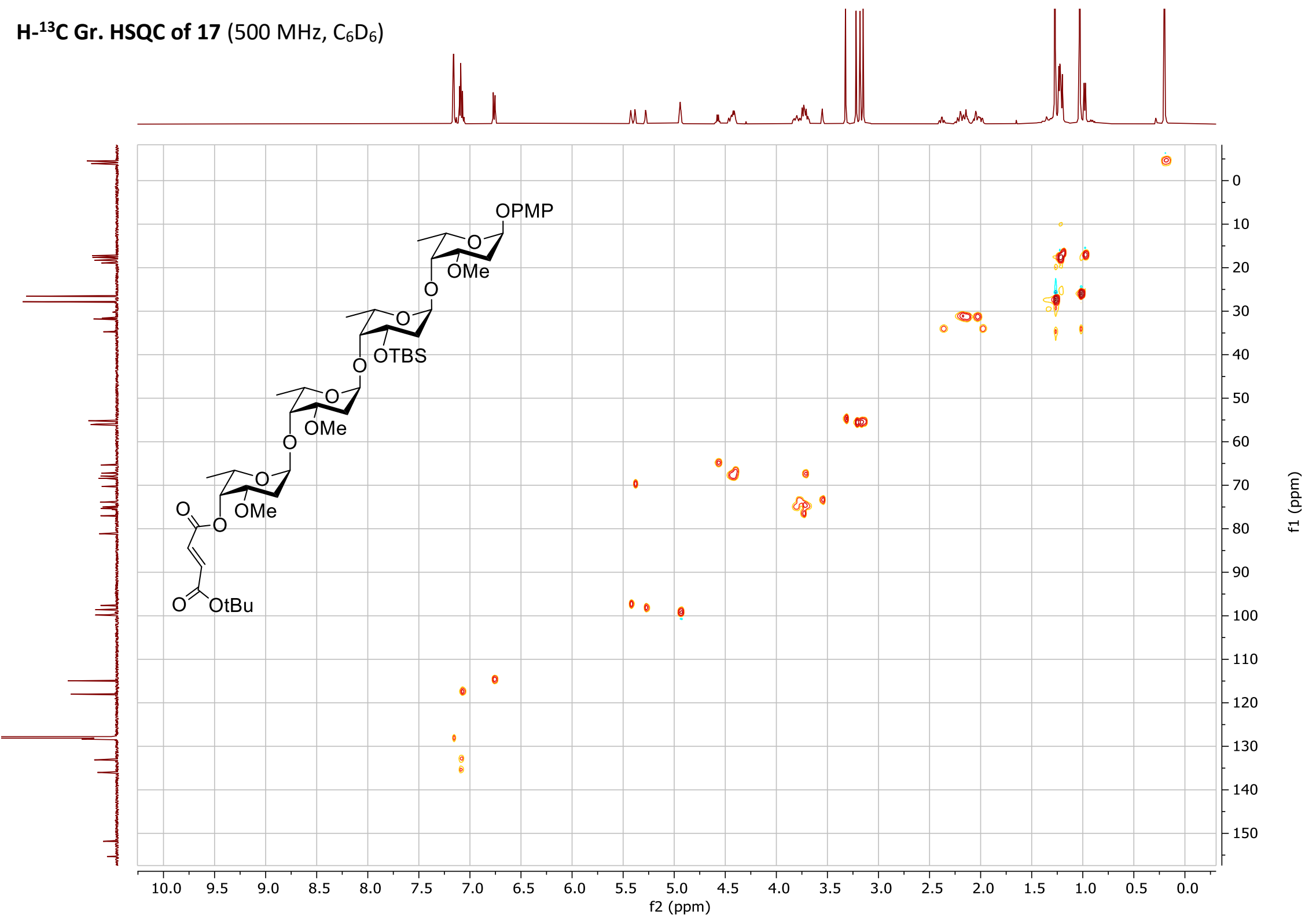
${ }^{1} \mathrm{H}-{ }^{1} \mathrm{H}$ Gr. cosY of $17\left(500 \mathrm{MHz}, \mathrm{C}_{6} \mathrm{D}_{6}\right)$
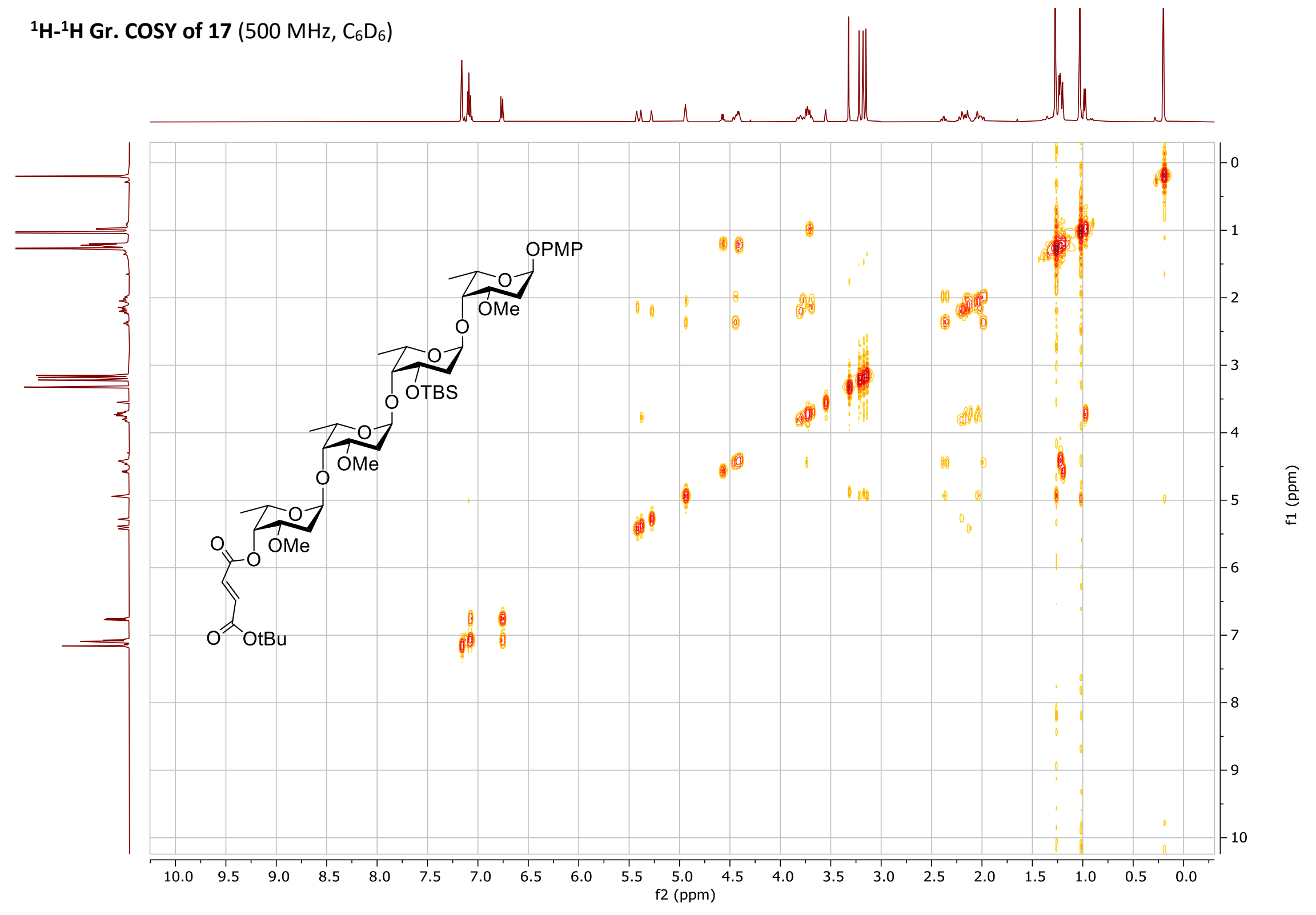


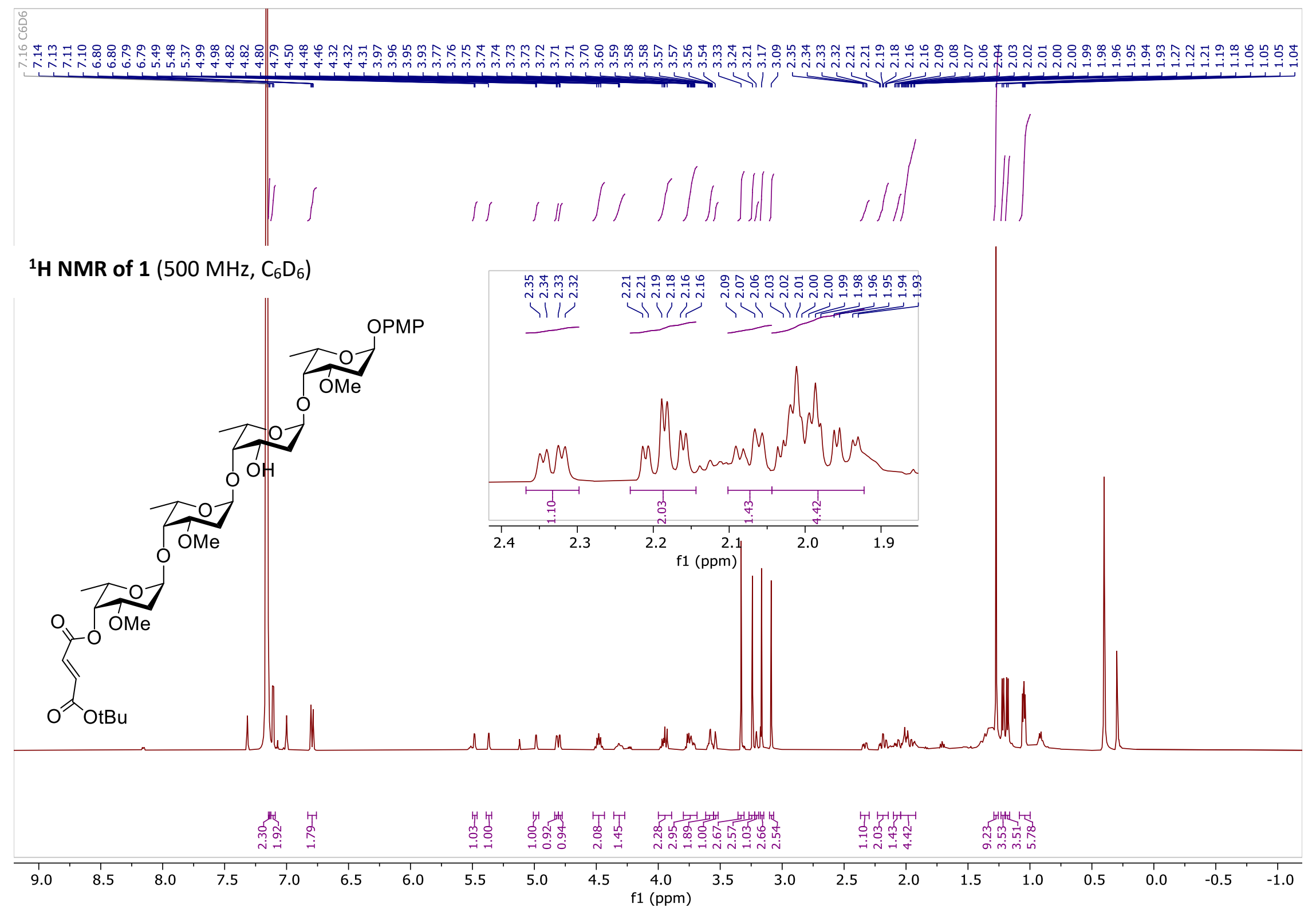




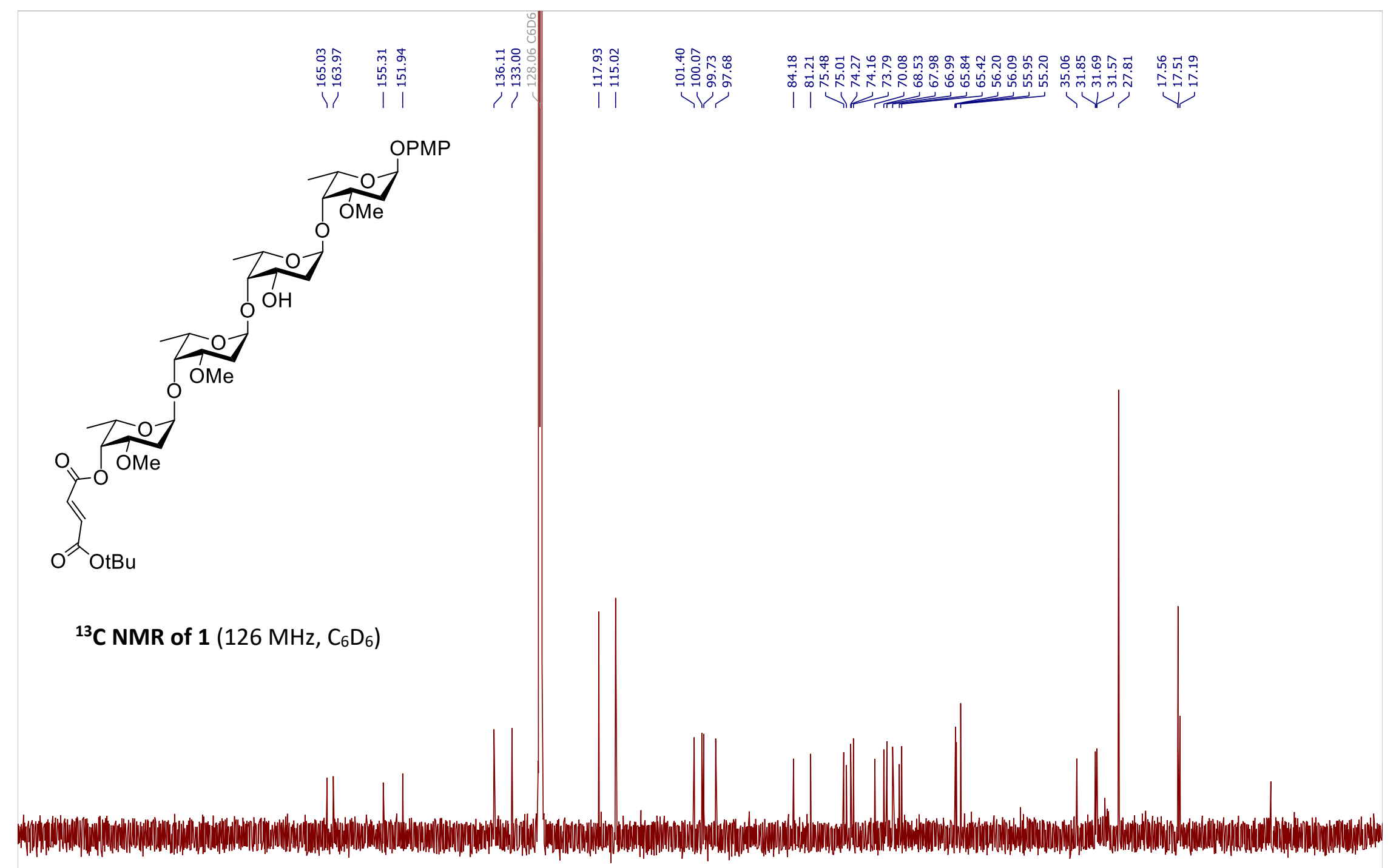

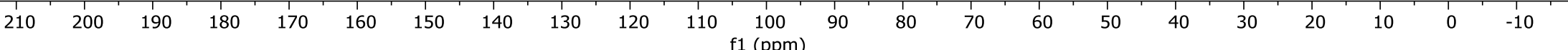




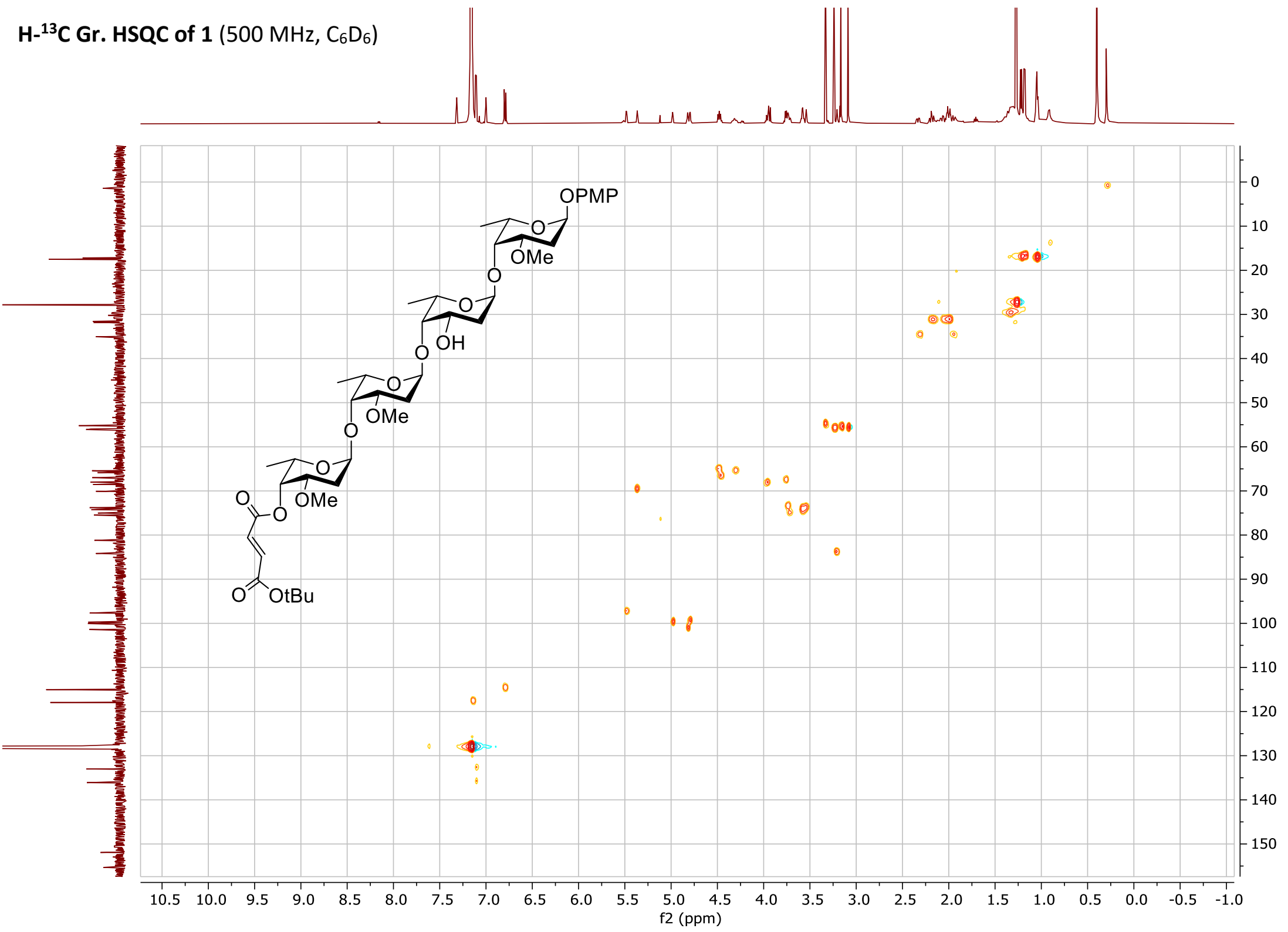


${ }^{1} \mathrm{H}^{-1} \mathrm{H}$ Gr. CosY of $\mathbf{1}\left(500 \mathrm{MHz}, \mathrm{C}_{6} \mathrm{D}_{6}\right.$ )
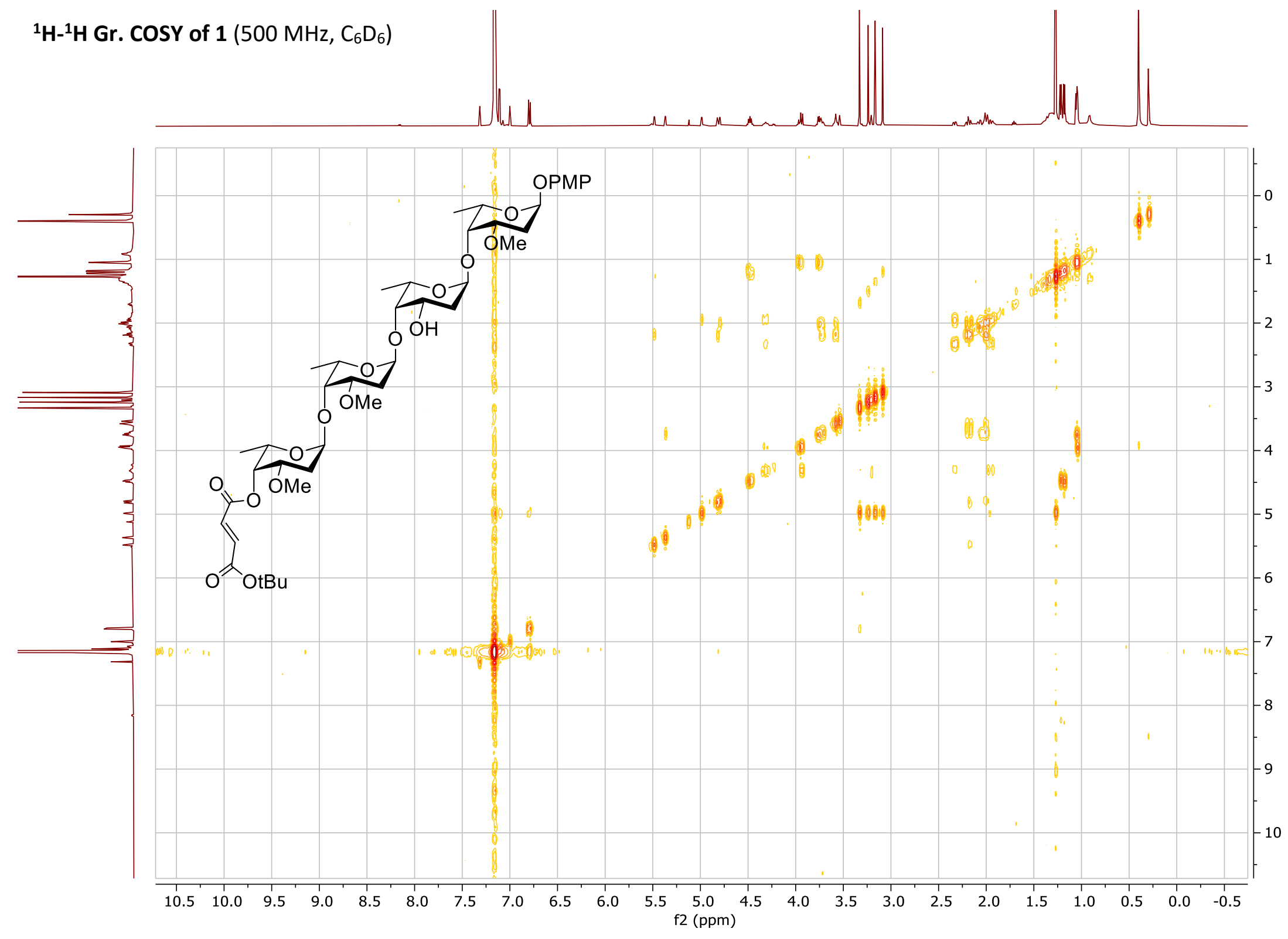